Distribution Category: Nuclear Waste Management (UC-70)

ANL $-81-53$

SILL- $-81-53$

DEE2 014374

ARGONNE NATIONAL LABORATORY

9700 South Cass Avenue

Argonne, Illinois 60439

FUEL-CYCLE PROGRAMS

QUARTERLY PROGRESS REPORT

April-June 1981

by

M. J. Steindler, Seymour Vogler, G. F. Vandegrift, Jacqueline Williams, T. J. Gerding, L. J. Jardine, J. K. Bates,

J. E. Kincinas, W. J. Mecham, G. T. Reedy, M. G. Seitz,

R. A. Couture, N. M. Levitz, T. F. Cannon, P. G. Deeken,

R. J. Meyer, E. T. Kucera, D. L. Bowers, R. E. Nelson,

J. E. Parks, L. E. Trevorrow, C. G. Wach, I. O. Winsch, J. E. Fagan, D. G. Graczyk, Henry Lautermilch, and R. E. Brock

Chemical Engineering Division

March 1982

Previous reports in this series

ANL-81-35

January-March 1981

ANL-81-13

ANL $-80-114$

October-December 1980

ANL-80-92

July-September 1980

Apri1-June 1980 


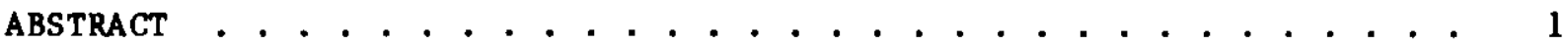

SUMMARY ............................. 2

I. DEVELOPMENT OF INTERIM HIGH-LEVEL WASTE FORMS . . . . . . . . 6

A. Introduction .................... 6

B. Experimental Studies on Interim Waste Forms . . . . . . . 6

1. Fused Salt . . . . . . . . . . . . . . . 6

2. Phosphate Immobilization .............. 7

C. Stability of $\mathrm{FePO}_{4}$. . . . . . . . . . . . . . . . 14

D. Interim Waste Form Development: Materials Studies . . . 15

1. Sintered Silicate/sludge System . . . . . . . . 16

2. Cement-silicate System ............... 18

I I. NEUTRON ACTIVATION AND TRACER STUdIES . . . . . . . . . . 20

A. Intzoduction . . . . . . . . . . . . . . . . 20

B. Qualification of Radioactive Tracer Method . . . . . . . 20

1. Introduction .................. 20

2. Radioact ive Glass Preparation and Source Counting . . . . 21

3. Leach ing Characterizations . . . . . . . . . . . . 22

4. Auxiliary Leach Studies... . . . . . . . . . . 37

III. BRITTLE FRACTURE STUDIES . . . . . . . . . . . . . . 54

A. Introduction . . . . . . . . . . . . . 54

B. Comparative Impact Testing of Alternative High Level

Waste Forms: Generation of Respirable Sizes . . . . . . . 54

1. Introduction ... . . . . . . . . . . . . 54

2. Experimental zrocedure .............. 55

3. Preparation of Test Specimens . . . . . . . . . . . . 56

4. Degcription of Materials Tested . . . . . . . . . . 56

5. Discussion of Results................ . 63

C. Methodology Development . . . . . . . . . . . 65

1. Background . . . . . . . . . . . . . . 65

2. Evaluation of Measurement of Size Distributions of Impact-Fracture Particulates . . . . . . . . . . 65

3. Correlation of Lognormal Particle Size Parameters with Input Energy Density . . . . . . . . 71

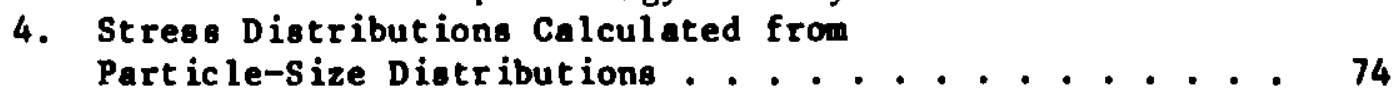

5. Energy Part it ioning in Impact Teat Devices...... . 77 
IV. FlUIDS IN ROCK ........................ 83

A. Repository-Analog Experiments . . . . . . . . . 83

B. Water for the Analog Experiment . . . . . . . . . . 83

C. Well Logging . . . . . . . . . . . . . . . . 83

D. Experimental Series.................. 85

V. TRACE-ELEMENT TRANSPCRT IN LITHIC MATERIAL BY FLUID FLOW . . • 86

A. Introduction .................... 86

B. Origin and Significance of Brine . . . . . . . . . . 85

C. Elution of Tritium through the Core . . . . . . . . . . 87

D. Improved Sampling Techniques . . . . . . . . . . . . 89

1. Tracers . . . . . . . . . . . . . . . . 89

2. Elution by Gas or Immiscible Liquid . . . . . . . . . . 89

3. Porosity Measurement and Leaching . . . . . . . . . . 89

E. Drying of Samples . . . . . . . . . . . . . . . . 89

F. Migration during Intrusive Emplacement . . . . . . . . . 90

VI. LIGHT WATER BREEDER REACTOR PROOF-OF-BREEDINC ANALYTICAL SUPPORT PROJECT . . . . . . . . . . . . . . . . 92

A. Full-Scale Shear ................ . . 92

B. Single Unit Dissolver.................. 93

C. Multiple-Dissolyer System . . . . . . . . . . . . 94

D. Scrap and Waste . . . . . . . . . . . . . . . . 94

E. Computer System . . . . . . . . . . . . . . . . 96

1. Hardware . . . . . . . . . . . . . . . 96

2. Software . . . . . . . . . . . . . . . 96

F. Error Analysis . . . . . . . . . . . . . . . . 97

G. Anslytical .................... 97

REFERENCES ........................... 98 
1. Typical Dissolution/Disintegration Experiment, Exp. 210-48, Simulated NFS Fused Salt Sample ............... . 10

2. Salt Block: Rate of Dissolution/Disintegration vs. Surface Area . . . . . . . . . . . . . . . . . . . . .

3. Phosphate-Immobilized Simulated NFS Waste:

Dissolution/Disintegration Rate vs. Surface Area . . . . . . . . . 12

4. Sintered Density of SS-65 and SS-C Specimens as a

Function of Compacting Pressure during Fabrication and Sludge Content of the Specimens . . . . . . . . . . . . 16

5. Fracture Strength, as Measured by Diametral Compression Technique, of Sintered SS-65 and SS-C Specimens as a Function of Compacting Pressure during Fabrication and Sludge Content of the Specimens . . . . . . . . . . . . . .

6. Measured Absolute Photopeak Efficiencies of Germanium Detector as a Function of Gamma Energy and Source-to-Detector Distances for Standards Given in this Text . . . . . . . . . . . 21

7. Summary of Weight Loss Data for Simulated Waste Glasses . . . . . 23

8. Preliminary Leach Test Results for Cold SRL $211^{\star \star}$ Glass . . . . 31

9. Preliminary Leach Results for Activated SRL $211^{\star \star \star}$ Glass . . . . . 32

10. Lognormal Plot of Cumulative Measured Weight Percent vs. the Measured Particle Diameter or Mesh Size for SRI

Frit 131 Glass (2) Impact Test . . . . . . . . . . . . . .

11. Lognormal Plot of Cumulative Measured Weight Percent vs. the Measured Particle Diameter or Mesh Size for PNL

76-68 Glass Impact Test . . . . . . . . . . . . . . . .

12. Lognormal Plot of Cumulative Measured Weight Percent vs. the Measured Particle Diameter or Mesh Size for MSD

SYNROC (1) Impact Test....................

13. Lognormal Plot of Cumulative Measured Weight Percent ve. the Measured Particle Diameter of Mesh Size for MSD

SYNROC (2) Impact Test .....................

14. Lognormal Plot of Cumulative Measured Weight Percent vo. the Measured Particle Dianeter or Mesh Size for CU High-Silica Impact Test 


\section{LIST OF FIGURES (contd)}

No.

Title

$\underline{\text { Page }}$

15. Lognormal Plot of Cumulative Measured Weight Percent vs.

the Measured Particle Diameter or Mesh Size for

Pyrex Impact Test . . . . . . . . . . . . . . . . . . . .

16. Lognormal Plot of Cumulative Measured Weight

Percent vs. the Measured Particle Diameter or Mesh

Size for Coal Impact Test ...................

17. Comparison of Lognormal Size Distribution for Eight

Impact Tests at $10 \mathrm{~J} / \mathrm{cm}^{3}$ as Described in the Text . . . . . . . 64

18. Illustration of Shape Effect on Particle Size Distribution

when Measured by Coulter Counter and Sieves . . . . . . . . 70

19. Illustration of Lognormal Size Parameters in Relation

to Measured Partic le Size Diatributions . . . . . . . . . . 71

20. Volume Distribution of Stress and Energy Density

for Sample of SRL 131 Waste Glass . . . . . . . . . . 76

21. Volume Distribution of Surface Area

and Number of Binary Cleavage Stages . . . . . . . . . . . 77

22. Model of Impact Test Device . . . . . . . . . . . . 78

23. Composition of Groundwater and Pore

Water from Precambrian Granite . . . . . . . . . . . . . . . . . 87

24. Elution of Tritium from Granite Core.............. 88 
1. Dissolution/Disintegration Rate Constants . . . . . . . . 13

2. Water Absorption during the Humidity Treatment; the Fracture Strength of Specimens Containing Synthetic Sludge, Sodium Silicate, and $\mathrm{Ca}(\mathrm{OH})_{2}$...................

3. Nominal Compositions of Simulated SRL Waste Glasses Ised in this Work

4. Summary of Test Conditions, Leachate Analytical Results, and Weight Loss Rates for SRL $211^{\star \star} \mathrm{Glass}$ under Study . . . . . . . . 24

5. Summary of Normalized Leach Rates Calculated from Data in Table 4 for SRL $211^{\star \star}$ Glass ............ 27

6. Summary of Test Conditions, Leachate Analytical Results, and Weight Loss Rates for SRL $211^{\star \star \star}$ Glass............ 33

7. Summary of Normalized Leach Rates Calculated from Data in Table 6 for SRL $211^{\star \star \star} \mathrm{Glass}$............ 35

8. Summary of Test Conditions, Leachate Analytical Results, and Weight Loss Rates for SRL 131 Glass under Study . . . . . . . . 38

9. Summary of Normalized Leach Rates Calculated from Data in Table 8 for SRL $131 \mathrm{Glass}$................. 40

10. Test Conditions and Leach Results for SYNROC Samples....... 41

11. Sol id SYNROC Sample Analyses ................. 52

12. Nominal Compositions of Alternative Waste Forms Used in th is Comparat ive Impact Study . . . . . . . . . . . . . 57

13. Reopirable Particles Generated in Comparative Impact Tests . . . 58

14. Compounds Used to Formulate Solution Similar to the Groundwater in the Grande Ronde Formation . . . . . . . . . . .

15. Concentrations of Constituents in Aqueous Solution and in Groundwater of the Grande Ronde Formation . . . . . . . . . . 
FUEL CYCLE PROGRAMS

QUARTERLY PROGRESS REPORT

April-June 1981

by

M. J. Steindler, Seymour Vogler, G. F. Vandegrift,

Jacquel ine Williams, T. J. Gerding, L. J. Jardine, J. R. Bates,

J. E. Kinc inas, W. J. Mecham, G. T. Reedy, M. G. Seitz,

R. A. Couture, N. M. Levitz, T. F. Cannon, P. G. Deeken,

R. J. Meyer, E. T. Kucera, D. L. Bowers, R. E. Nelson,

J. E. Parks, L. E. Trevorrow, C. G. Wach, I. O. Winsch,

J. E. Fagan, D. G. Graczyk, Henry Lautermilch, and R. E. Brock

\section{ABSTRACT}

A program continues for the development of an interim waste form capable of being transported from facilities where waste is generated to terminal waste processing. In work on a process for converting high level liquid waste to a phosphate-immobilized solid waste and heating the mixture to dryness $\left(400^{\circ} \mathrm{C}\right)$, the amount of phosphate required to be added to $\mathrm{simulated}$ waste has been found to be $233 \%$ of the theoretical amount to balance the cationic charge as $\mathrm{PO}_{4}^{3-}$.

Rates of dissolution (i.e., penetration rates) of various interim waste forms (Buch as Bynthetic waste salt with and without synthet ic sludge, phosphate-immobilized specimens, and silicateagglomerated $\mathrm{Fe}_{2} \mathrm{O}_{3}$ ) in stagnant water ranged from $1 \times 10^{-5}$ to $1 \times 10^{-4} \mathrm{~cm} / \mathrm{s}$.

For sintered silicate/sludge systems, compacts having the desired mechanical strength were obtained by using a maximum of 30 wt $Z$ sludge and compact ion at $>211 \mathrm{MPa}$. In other experiments, it was observed that pellets of adequate strength were prepared from mixtures of 75 wt $\%$ sludge, 20 wt $\%$ sodium silicate, and 5 wt $7 \mathrm{Ca}(\mathrm{OH})_{2}$; these specimens showed no weight 1088 upon immers ion in water.

Measurements of rates of leaching from 8 imulated waste glasses are continuing. The leach rates of different elements from the same glasses sometimes differ by several orders of magnitude so that no single analytical technique appears to be optimum for all elements. Leach tests on over 90 SYNROC and irradiated glass samples have been completed.

Laboratory-scale impact tests of solid alternative wate forms are being performed ( 1 ) to assess source terms of airborne releave (from particle size distributions) and (2) to assess the increases in source terms of leach rates (from increases in ourface area). From this, dispersion from mechanical impacts during normal and 
accidental conditions of transport can be determined. Cylindrical specimens (ceramics, frits, and glass) were impacted diametrally with a striker bar. Particle size measurements were made, and plots show that size distributions are lognormal in the range, $10 \mathrm{~mm}$ to $2 \mathrm{~mm}$.

A review of the methodology under development for the characterization of the impact behavior of brittle materials included the following topics: evaluation of measurements of particle size distributions; the relation of energy and stress distributions to particle-size distributions; and energy partitioning in impact testing mach ines.

Logging techniques are being developed to measure the relative amount of residual oil in a depleted oil reservoir by inject ing gamma-active solution into it. For planned laboratory-scale experiments, equipment has been collected, sandstone samples acquired, and an aqueous solution prepared having a composition similar to that of brine reservoir fluid.

The flow of groundwater through igneous rocks is of considerable interest in connection with geologic storage of high-level radioactive waste. Analyses of samples from the Notch Peak (House Range, Utah) intrusive body suggest that groundwater flow was through fractures and not through the rock matrix, indicating that no new processes of migration need be considered in the design of a nuclear waste repository.

Test ing and development of equipment for the destructive analys is of full-length irradiated fuel rods from the LWBR is in progress. This work is in support of the LWBR Proof-of-Breeding Project.

\section{SUMMARY}

\section{Development of Interim High-Level Waste Forms}

Continued experimentat ion on phosphate immobilization (i.e., the use of phosphate as a binder to form an interim waste suitable for shipping to a terminal processing site) has indicated that to consistently prepare a high-density, glassy, structural ly strong product, the amount of phosphoric acid added to the $s$ imulated NFS waste must be equal to or greater than 2337 of the theoretical amount to balance the cationic charge as $\mathrm{PO}_{4}^{3-}$. In experiments run with $233 \%$ phosphate, the addition of water during distillation is required to help remove nitric acid as ater/nitric acid azeotrope.

The rates of dissolution of various interim waste forms in stagnant water were messured. Massive pieces of synthet ic Nuclear Fuel Services (NFS) waste salt with or without 8 wt 7 synthet ic sludge yielded a penetration rate of about $4 \times 10^{-4} \mathrm{~cm} / \mathrm{s}$. Penetration rates for phosphate-immobilized specimens from different preparations varied from $6 \times 10^{-5}$ to $1 \times 10^{-4} \mathrm{~cm} / \mathrm{s}$. Two samples of $\mathrm{sil}$ icate-agglomerated $\mathrm{Fe}_{2} \mathrm{O}_{3}$ had penetration rates of $1 \times 10^{-5}$ to $2 \times 10^{-5} \mathrm{~cm} / \mathrm{s}$ in stagnant water and were the least reactive materials in otagnant water. 
Preliminary results on the hydrolysis of $\mathrm{FePO}_{4}$ suggest that instead of $\mathrm{FePO}_{4}$, previously reported to be present in the NFS waste tank, ferric ion is probably present as hydrated $\mathrm{Fe}_{2} \mathrm{O}_{3}$.

Testing of sintered silicate/sludge systems indicated that SS-65 and SS-C compacts containing $>50$ wt $\%$ sludge were mechanically extremely weak (strength $0-0.5 \mathrm{MPa}$ ). Only SS-C compacts containing 30 wt \% sludge and compacted at $\geq 211 \mathrm{MPa}$ exhibited the desired mechanical strength.

Studies were continued on the cementitious bonding of sodium silicatecalcium hydroxide-synthetic sludge systems. Cold-pressed samples are exposed to a controlled high-humidity air ( $90 \%$ relative humidity) for $24 \mathrm{~h}$ at $35^{\circ} \mathrm{C}$. Samples thus prepared and containing 75 wt \% sludge, 20 wt \% Jdium silicate, and 5 wt $\% \mathrm{Ca}(\mathrm{OH})_{2}$ yielded pellets of sufficient strength. Such a specimen showed no detectable weight loss when immersed in stagnant water at room temperature.

Neutron Activation and Tracer Studies

Comparative MCC-1 type leach rate measurements of SRL frit 211 and SRL frit 131 simulated waste glasses are in progress. Conventional solution analytical methods (coiorimetry, ICP and DCP emission spectroscopy, atomic absorption), neutron activation techniques, and radioactive tracers are all being employed to measure the leach rates of about seventeen different elements in these glasses. Leach rates of different elements from the same glasses are observed to sometimes differ by several orders of magnitude (i.e., Eu 《 Cs) so that no single analytical technique appears to be optimum for all elements. Many analyses are in progress.

Significant protective layers which retard leaching are observed on SRL glasses. These layers, studied using electron microprobe and scanning electron microscopy techniques, we re found to be depleted in sodium and silicon and enriched in manganese, magnesium, and rare earths. These observations correlate well with solution analysis results.

Leach tests on over 90 SYNROC and irradiated glass samples have been completed and the results obtained. For SYNROC leaching in Teflon and polypropylene bottles, there seems to be a container effect whereby the leach rates of SYNROC in polypropylene are one-third those in Teflon.

Brittle Fracture Studies

Seven additional impact tests were made using a standardized test procedure, and the results are compared with similar tests of simulated alternat ive waste materials reported in the previous report period. In all tests, cylindrical specimens of about $2.5 \mathrm{~cm}$ by $2.5 \mathrm{~cm}$ were impacted diametrally with a 10-kg steel striker bar that fell from a height predetermined to produce a maximum energy input of $10 \mathrm{~J} / \mathrm{cm}^{3}$ of material. Particle size measurements made by both sieving and Coulter councer analysis in the range of $5 \mu \mathrm{m}$ to $2 \mathrm{~mm}$ were used to determine the lognormal size parameters which describe the complete size distribution of all particles smaller than $2 \mathrm{~mm}$; this fraction contains more than $95 \%$ of the total geometric surface area formed by fracture, as estimated from the lognormal size parameters. The respirable size fractions of PNL 76-68 glass, SYNROC ANL, LLL SYNROC B, LLL 
SYNROC D, and SRL frit 131 glass were very similar; respirable fractions constituted 0.10 to 0.18 wt \% of the particles. In a test made to allow comparison, a specimen of high-silica glass from Catholic University showed a respirable fraction of 0.22 wt \%, which is similar to the result of 0.27 wt \% obtained for a Pyrex specimen. The above results are with in the range of respirable fractions previously reported for Rockwell TAILORED CERAMIC $(0.05$ wt $\%$ ) and ORNL FUETAP concrete $(0.43$ wt \%). Replicate tests were done for SRL $131 \mathrm{glass}$ and SYNROC MSD. The results for each material agreed within the standard error of the regression analysis for the respirable fraction and the lognormal parameters.

A review of the methodology under development for the characterization of the impact behavior of brittle materials included the following topics: evaluation of measurements of particle size distributions; the relation of energy and stress distributions to particle-size distributions; and energy partitioning in imact testing mach ines.

Fluids in Rock

Reported are preparations to test the performance of proposed nuclearwaste repositories and to develop procedures for core-flood experiments. The core-flood experiments will be used to examine proposed methods of logging the oil in depleted oil reservoirs. The proposed logging methods use gammaactive fluid injected into the reservoir to detect the presence of oil.

Trace-Element Transport in Lithic Material by Fluid Flow

It was previously reported that $\mathrm{NaCl}-\mathrm{CaCl}_{2}$ brine $([\mathrm{Cl}-]>0.24 \mathrm{M})$, wh ich has a very $h$ igh $F^{-}$content $(>3.3 \mathrm{mM})$, was eluted from granite from Northern Illinois drill hole UPH-3. This brine is similar in composition, and is believed to be of similar origin, to brine of sedimentary origin from the Illinois Basin. The coexistence of calcium and fluoride in the water implies the presence of a complexing agent, most likely boron.

The absolute concentrations of ions in the pore water were not determined because of ambiguity in the effective pore volume. Elution of tritiumlabeled water through the core shows that the effective porosity is at most $0.07 \%$. Three improved sampling techniques, which promise to give information on absolute concentrations, are discussed.

Preservation of samples is discussed. It is estimated that granite core $4.8 \mathrm{~cm}$ in diameter may dry out in as little as 28 days.

Work on the Notch Peak intrusive body is also summarized. The evidence indicates that there was no perceptible migration of major elements or trace elements, except possibly for rubidium, from the Notch Peak, Utah, monzonite intrusion into surrounding limestones and siltstones, except at skarns. The evidence suggests that nearly all flow was directed through fractures, and not through the rock matrix. 
Light Water Breeder Reactor Proof-of-Breeding Analytical Support Project

This Project is responsible for the destructive analysis of full-iength irradiated fuel rods from the LWBR. The results will be used by the Bettis Atomic Power Laboratory (BAPL) in support of their nondestructive assay of the end-of-life (EOL) core to determine the extent of breeding.

Activity is reported on four main subactivities of this Project: (1) the full-scale shear (FSS), (2) the single-unit or prototype dissolver (SUD), (3) multiple dissolvers needed for the destructive analyses of full-length (about 3-m) irradiated fuel rods for the EOL campaign, and (4) scrap/waste disposal. In addition, current work by the Analytical Section in support of this Project is being done in the areas of planning and procurement. Development of an integrated computer/data acquisition system for the entire Project is also in progress. 
I. DEVELOPMENT CF INTERIM HIGH-LEVEL WASTE FORMS

(Seymour Vogler, G. F. Vandegrift, Jacqueline Williams,

E. T. Kucera, ${ }^{\dagger} T_{*} J$. Gerding, George Bandyopadhyay, ${ }^{\star}$

S. M. Gehl, ${ }^{\star}$ His ao Yamada, ${ }^{\star}$ T. M. Galvin, ${ }^{\star}$

J. E. Slattery, ${ }^{\star}$ W. J. Grajek, ${ }^{\star}$ and R. B. Poeppe ${ }^{\star}$ )

\section{A. Introduction}

High-level liquid radioact ive wastes (HLLW) are often produced at facilities in which terminal processing of the waste is not practical because of the high cost or hazards involved in such an operation. Under these circumstances, the waste could be incorporated into an interim solid waste form for shipping to a terminal processing site. The interim form should require only simple processing methods, so that extensive additional facilities will not be required at the originating site. Additionally, the interim waste form must sat isfy shipping criteria, particularly in relation to transportation accidents; should be mechanically and chemically stable in the ambient environment; and should be readily compatible with the terminal-waste-form processes. From this research, there should evolve a technology for preparing interim waste forms compatible with transportation requirements and with subsequent terminal waste form processing.

B. Experimental Studies on Inter im Waste Forms

Seymour Vogler, G. F. Vandegrift, T. J. Gerding, E. T. Kucera, ${ }^{\dagger}$ and Jacqueline Willians)

Studies were continued on two possible interim waste forms (fused salt and phosphate-immoblized waste) that may be suitable for solidifying the HLLW at the Nuclear Fuel Services (NFS) Plant at West Valley. The fused salt form would be prepared by mixing the supernate and sludge constituents of NFS vaste, evaporating away it $s$ water content, and malt ing the low-melting $\mathrm{NaNO}_{3} / \mathrm{NaNO}_{2} /$ $\mathrm{Na}_{2} \mathrm{SO}_{4} / \mathrm{NaOH}$ salts while suspending the nonmelt ing oxides in this melt. The cooled solid suspension product would be shipped and stored.

A phosphate-immobilized interim waste form would be prepared by adding $\mathrm{H}_{3} \mathrm{PO}_{4}$ to the supernate sludge mixture, followed by a distillation progressing to about $400^{\circ} \mathrm{C}$. As the distillation proceeds (i.e., as the temperature rises), the distillate would change from $\mathrm{H}_{2} \mathrm{O}$ to $\mathrm{H}_{2} \mathrm{O} / \mathrm{HNO}_{3} / \mathrm{HCl}$ and back to $\mathrm{H}_{2} \mathrm{O}$ aga in as all traces of nitrate, nitrite, and chloride would be removed from the waste and replaced with phosphates, pyrophosphates, and metaphosphates. The product is not a glass (much higher temperatures, $850-1000^{\circ} \mathrm{C}$, would be necessary for this) but a glasslike homogeneous solid solution of mostly sodium (plus other cationic) pyro- and metaphosphates.

\section{Fused Salt}

Studies are under way to measure the resistance of the fused salt (basically $\mathrm{NaNO}_{3}, \mathrm{NaNO}_{2}$, and $\mathrm{Na}_{2} \mathrm{SO}_{4}$ ) to irradiat ion. These studies are necessary to ensure that storage of NFS waste in this form for up to $20 \mathrm{y}$ will

\footnotetext{
* Member of the Materials Science Division, Argonne National Laboratory. ${ }^{\dagger}$ Member of the Analytical Chemistry Laboratory, Chemical Engineering Division.
} 
not unduly pressurize it 3 sealed containment canisters. Literature informa$t$ ion [JOHNSON-1961, -1970] indicates that sodium nitrate is decomposed when exposed to gamma radiation:

$$
\mathrm{NaNO}_{3} \mathrm{O} \rightarrow \mathrm{NaNO}_{2}+\frac{1}{2} \mathrm{O}_{2}
$$

There is no information, however, to indicate the response to gamma irradiation of (1) a $70 \% \mathrm{NaNO}_{3} / 30 \% \mathrm{NaNO}_{2}$ salt alone or (2) a similar mixture in which the sludge components of NFS waste are suspended. Our studies will measure the effects of these additional chemical components on the irradiation of $\mathrm{NaNO}_{3}$.

In studies completed thus far, the ability of our equipment and techniques to duplicate the literature $g$ value for reaction 1 has been tested and verified. These studies will be reported in more detail when they are completed.

\section{Phosphate Immobilization}

HLLW converted to a phosphate solid appears to be an attrictive interim waste form. Phosphate, pyrophosphate, and metaphosphate ions form strong bonds with most metal ions, and their salts with multivalent metal cations have low aqueous solubilities. Phosphate and acid phosphate cements, used as dental sements, have low leachability and high compressive strengths.

\section{a. Preparation of Phosphate-Immobilized Simulated NFS Waste}

Further experiments have been performed this month in the manner described previously [STEINDLER-1981C]. The procedure for preparing a phosphate-immobilized waste form is basically one of adding phosphoric acid to the liquid waste and heating the mixture to dryness $\left(400^{\circ} \mathrm{C}\right)$. After an amount of phosphoric acid greater than that necessary to balance the cationic charge as $\mathrm{PO}_{4}^{3-}$ is added to the waste, the resultant solution is heated. Nitrate and chloride are distilled away from the waste as azeotropic mixtures of $\mathrm{HNO}_{3}, \mathrm{HCl}$, and $\mathrm{H}_{2} \mathrm{O}$.

Three aspects of the experiments were studied:

- The effect of the ratio of phosphoric acid to total amount of cationic charge in the simulated NFS waste on the physical properties of the product.

- The effect of additional water, added to the distillation kettle at elevated temperatures, on driving off more of the $\mathrm{HNO}_{3}$ before the product solidifies.

- Testing our ability to duplicate experimental and product conditions. 
Results have indicated that to consistently prepare a highdensity, glassy, structurally strong product, the amount of phosphoric acid added to the simulated NFS waste must be equal to or greater than $233 \%$ of the theoretical amount to balance the cationic charge as $\mathrm{PO}_{4}^{3-}$. (We have done experiments in which 117 to $400 \%$ of the theoretical amount was added.) In experiments with $233 \%$ phosphate, water must be added during distillation to help remove $n$ itric acid as a water/nitric acid azeotrope. If there is still nitrate in the phosphate-iminobilized waste after its viscosity rises too high, the final product becomes very porous because $\mathrm{NO}_{2}$ gas is evolved during the thermal decomposition of nitrate. An experiment run with a phosphate rat io of $291 \%$ and with no water added during distillation showed slight signs of nitrate decomposition; the material. appeared to be more ceramic than glassy. When the amount of phosphate was raised to $389 \%$ and no water was added during distillation, the material was glassy. A preparation containing only $117 \%$ of the theoretical amount of phosphoric acid was extremely porous and of very low structural integrity.

\section{b. Rates of Dissolution of Possible Interim Waste Forms for NFS Wastes}

Studies of dissolution/disintegration rates in stagnant water have been completeu on four preparations of phosphate-immobilized simulated NFS waste. The specifics for each of these preparations are:

- 209-140. The phosphoric acid added was $233 \%$ of that needed to balance the cationic charge as $\mathrm{PO}_{4}^{3-}$. All of the phosphoric acid was added at one time before distillation started. Water was added incrementally during distillation. The solid product remaining after distillation was complete was gray glassy, nonporous, and homogeneous-looking with a density of $2.64 \mathrm{~g} / \mathrm{mL}$.

- 209-147. The phosphoric acid added was $233 \%$ of that needed to balance the cationic charge as $\mathrm{PO}_{4}^{3-}$. In this experiment, only $75 \%$ of the phosphoric ac id was added in itially; the remainder was diluted with water and added incrementally during distillation The solid product produced in this experiment was gray, extremely porous, homogeneous, ceramic-looking, and massive and had a density of $1.68 \mathrm{~g} / \mathrm{mL}$.

- 214-03. The phosphoric acid added was $291 \%$ of that needed to balance the cationic charge as $\mathrm{PO}_{4}^{3-}$. All of the $\mathrm{H}_{3} \mathrm{PO}_{4}$ was added before heat ing began, and no additional water was added during distillation. The massive product of this experiment was more ceramic than glassy. It appeared to be homogeneous and was basically ncnporous.

- 214-08. The phosphoric acid added was $400 \%$ of that needed to balance the cationic charge as $\mathrm{PO}_{4}^{3-}$. All of the $\mathrm{H}_{3} \mathrm{PO}_{4}$ was added before heating, and no additional water was added during distillation. The product was glassy and nonporous. It appeared to be homogeneous and had a light green tint to its gray color. 
For the sake of comparison, the dissolution/disintegration rates in stagnant water of other materials were also measured. These materials were:

- Salt Block Samples (obtained from M. G. Seitz, Argonne National Laboratory). This material, taken from the Mississippi Chemical Corp. potash mine in southern New Mexico, contained (in order of approximate wt \%) $\mathrm{NaCl}$, $\mathrm{KCl}$ (sylvite), $\mathrm{Fe}_{2} \mathrm{O}_{3}, \mathrm{~K}_{2} \mathrm{MgCa}_{2}\left(\mathrm{SO}_{4}\right)_{4} \cdot 2 \mathrm{H}_{2} \mathrm{O}$, and a host of other minerals.

- Simulated NFS Waste Fused Salt containing the concentrations of $\mathrm{NaNO}_{3}, \mathrm{NaNO}_{2}, \mathrm{NaOH}$, and oxides of other metals (except U), present in NFS waste.

- Fused Salt prepared to simulate the supernate only of NFS waste--63.4 $\mathrm{g} \mathrm{NaNO}_{3} / 27.2 \mathrm{~g} \mathrm{NaNO}_{2} / 17.5 \mathrm{~g} \mathrm{Na}_{2} \mathrm{SO}_{4} / 1.0 \mathrm{~g} \mathrm{KOH}$.

- Pellets of Silicate-Agglomerated $\mathrm{Fe}_{2} \mathrm{O}_{3}$ (prepared by G. Bandyopadhyay et al., Materials Science Division, ANL).

- A piece of Brach's Sparkler lemon-flavored hard candy.

Several samples of different geometries and therefore different surface areas were tested for each of several waste forms--the phosphateimmobilized simulated waste forms, the fused salt simulated waste form, and the salt block. The rates of dissolution/disintegration for these three materials could therefore be correlated rith initial surface areas.

\section{(1) Experimental}

Sample preparation varied from material to material; each is therefore discussed separately:

- Brach's lemon-flavored hard candy: used as taken froin the wrapper.

- Silicate-Agglomerated $\mathrm{Fe}_{2} \mathrm{O}_{3}$ : used directly in the pelletized form as received.

- Salt Block: a hacksaw and a file were used to cut rectangular samples.

- Simulated NFS Fused Salt: samples were prepared by heating the solid mixture of the salt and metal oxide mixture in a small furnace contained in a dry box. When the $\mathrm{NaNO}_{3} / \mathrm{NaNO}_{2}$ mixture melted, the suspension was swirled and poured into crucibles having various geometries. As the material cooled, a pipe formed down the center of these samples; additional material was then poured into these pipes to fill them or at least seal them. 
Phosphate-Immobilized Simulated NFS Waste: because this material acts like glass, simple geometric shapes are very difficult to prepare. It is too hard to cut with a hacksaw or to file. It also does not cleave very well. Consequently, only sharp corners could be remove ${ }^{1}$, and surface geometries of samples needed to be defined by as many as thirteen faces.

Before rate studies were started, the samples were weighed on a top-loading balance to the nearest $0.01 \mathrm{~g}$ and the surface areas were estimated. To estimate a surface area, the samples's surface was defined as a series of geometric planes (rectangles and triangles), and the length of each side of these planes was measured to the nearest millimeter with a ruler.

The equipment for measuring the dissolution/disintegration rates consisted of a $4 \mathrm{~L}$ beaker containing $4 \mathrm{~L}$ of distilled/deionized water, a stainless steel mesh basket tied (with Teflon string) to a hook located on the underside of a Mettler 1200 top-loading balance, and a timer capable of measuring seconds. Experiments were begun by placing the sample in the basket and the basket in the water. The timer was then started. (The weight of the empty basket had previously been tared.) The weight of the sample and the elapsed time were periodically recorded. Figure 1 is a plot of the weight of sample in water vs. time for a typical experiment.

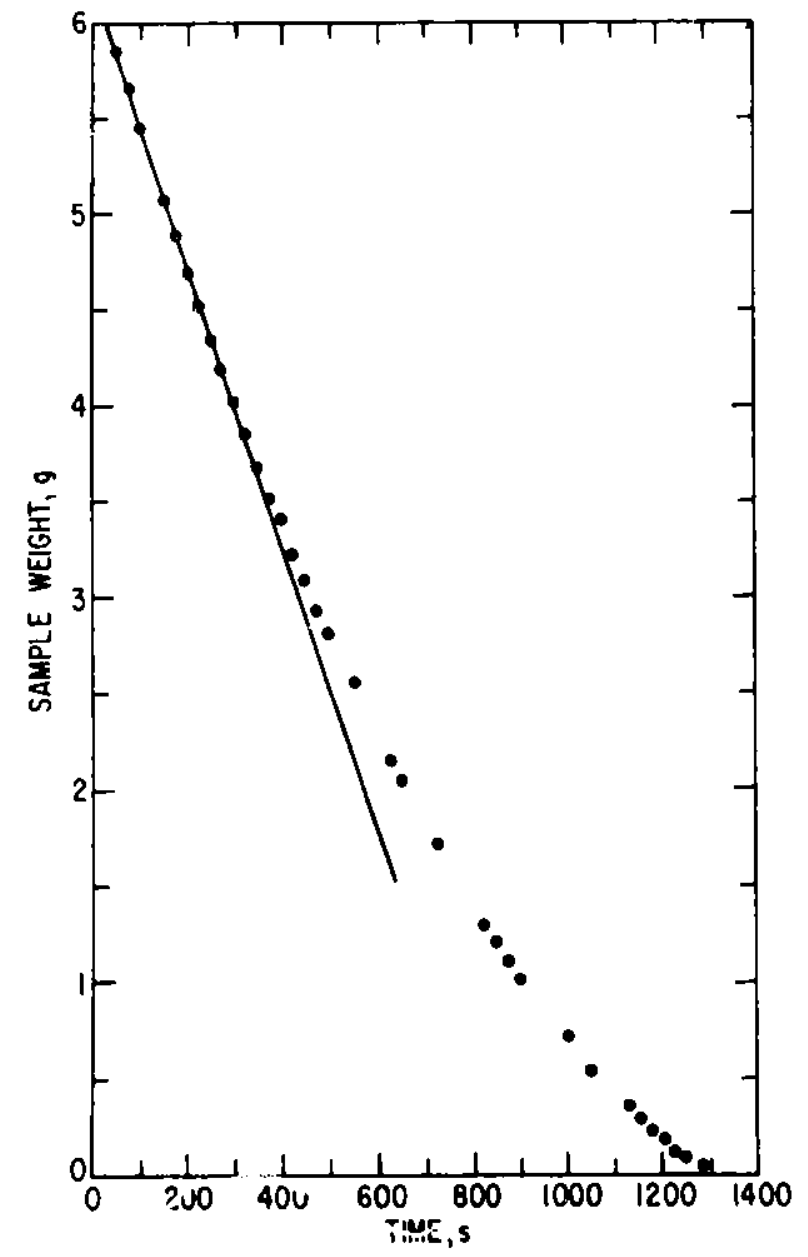

Fig. 1 .

Typical Dissolution/Disintegration Experiment, Exp 210-48, Simulated NFS Fused Salt Sample 
As would be expected, the shapes of these curves vary with the geometries of the dissolving/disintegating pieces. In general, the smaller the change in surface area with weight loss, the less the curve deviates from the initial. linear slope as the sample loses weight. The weight loss vs. time curve for a flat th in object, whose surface area shrinks only slightly with weight loss, is linear over a much wider range than for a cubic object.

For each experiment, a rate was measured as the negative slope at the initial linear portion of the curve. This rate, measured in $\mathrm{g} / \mathrm{s}$, was corrected for the buoyancy effect of water by the relationship:

$$
\text { Rate }_{\text {corr }}=\operatorname{Rate}\left(\frac{\text { sample we ight in air }}{\text { initial sample weight in water }}\right)
$$

Also, the densities of thse materials were derived by measuring the sample weights in air and in water:

$$
\text { density }=\frac{\text { weight in air }}{\text { weight in air }- \text { we ight in water }}
$$

\section{(2) Results}

The rate of dissolution/disintegration was measured as a function of surface area for seven materials--salt block procured from M. Seitz, simulated NFS fused salt wastes with and without sludge material, and four different phosphate-immobilized NFS waste forms. To give credence to the method, the salt block was tested first. The salt block was mostly soluble material but did contain a sizable amount (about $10 \%$ ) of $\mathrm{Fe}_{2} \mathrm{O}_{3}$, which has a very low solubility in water. This material has, therefore, the same type of soluble/insoluble material mix as the possible interim waste forms.

All materials tested showed a linear correlation of initial rate of dissolution/disintegration with surface area. Figures 2 and 3 are typical examples of the results.

In addition, dissolution/disintegration experiments were performed on two samples of $8 \mathrm{il}$ icate-agglomerated $\mathrm{Fe}_{2} \mathrm{O}_{3}$ that were prepared as described earlier [STEINDLER-1981B]. The first sample was SS-C/ $\mathrm{Fe}_{2} \mathrm{O}_{3}$ in a rat io of $2 / 1$ (SS-C is a $2 / 1$ weight rat io of $\mathrm{SiO}_{2} / \mathrm{Na}_{2} \mathrm{O}$ silicate powder). The second sample was prepared with a $6 / 1 \mathrm{ss}-\mathrm{C} / \mathrm{Fe}_{2} \mathrm{O}_{3}$ ratio. The weight loss vs. $t$ ime dependencies of these experiments were different from those of all other materials tested; there was an induction period of $20-30 \mathrm{~min}$ before weight loss began. After this induction period (when the samples not only did not lose weight but gained weight), these two samples behaved like the other materials.

To give a qualitative signifance to the dissolution/ disintegration rates of the above-mentioned materials, the dissolution rate of a piece of Brach's Sparkler lemon-flavored hard candy was measured. The dissolution of this material proceeded in the same manner as did the other materials even though all of this sample was soluble. 


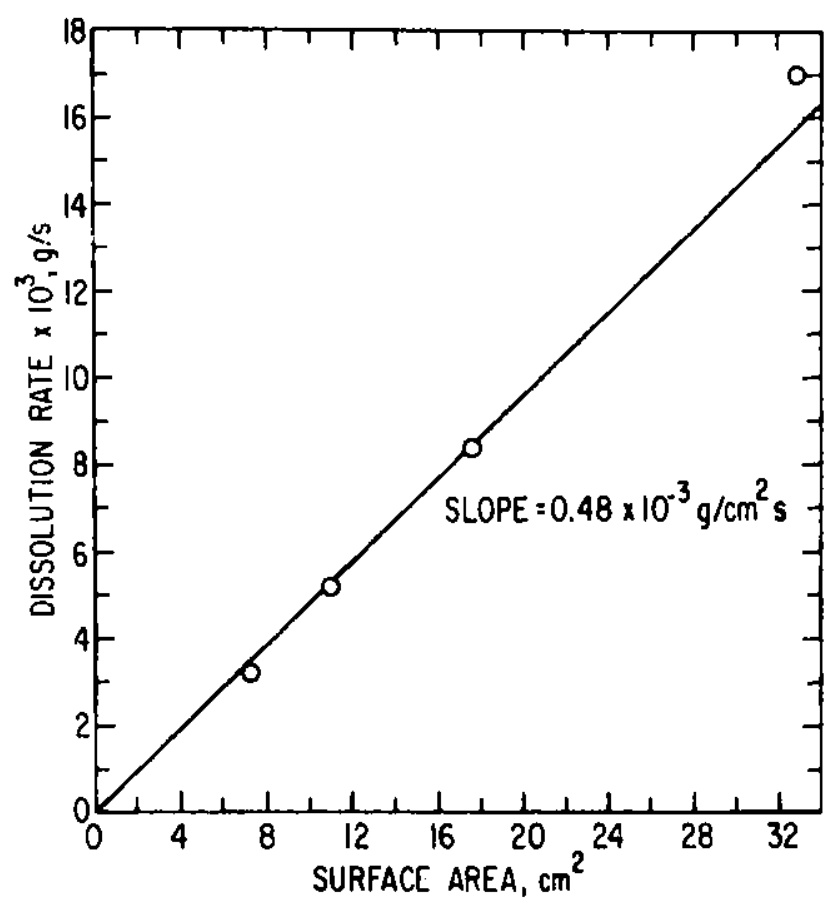

Fig. 2. Salt Block: Rate of Dissolutior/ Disintegration vB. Surface Area

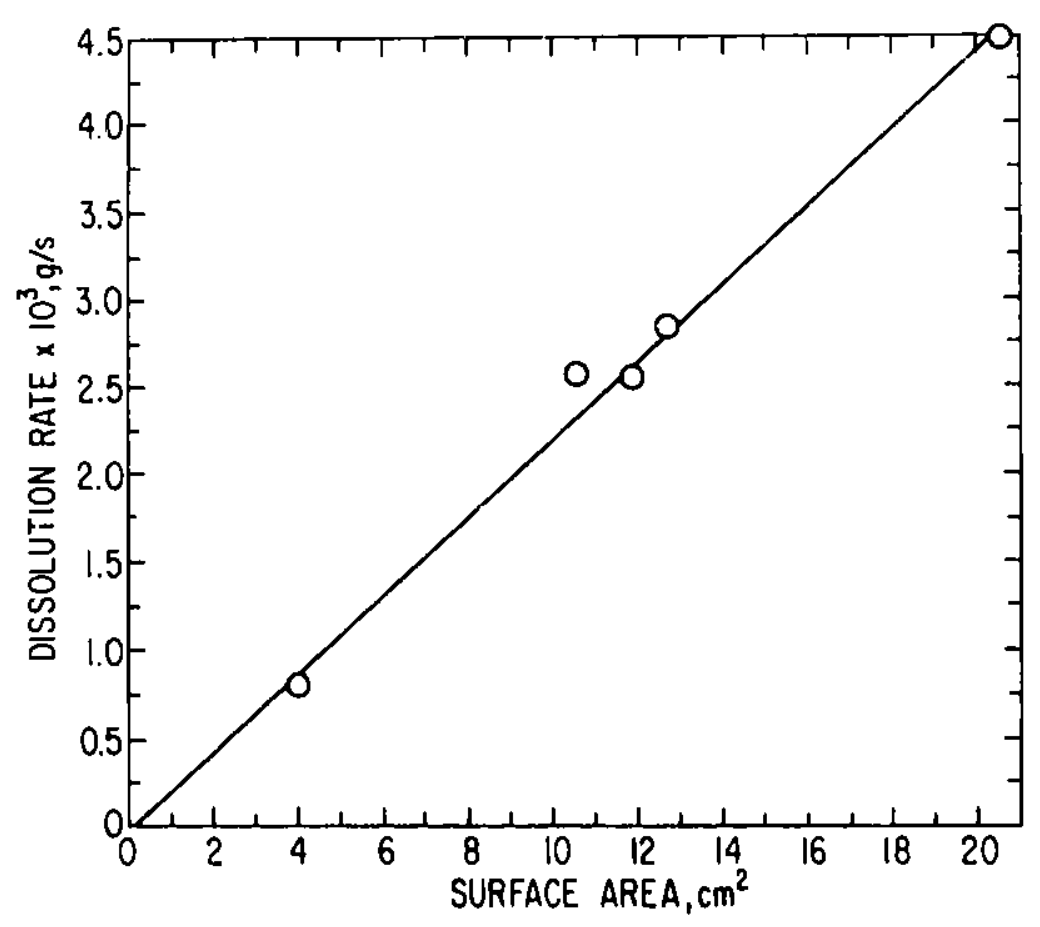

Fig. 3. Phosphate-Immobilized Simulated NFS Waste: Dissolution/Disintegration Rate va. Surface Area 
The rates of dissolution/disintegration for all of these materials (Table 1) may be compared. By dividing the rate of weight los a per $\mathrm{cm}^{2}$ by the density of the material, a penetration rate constant in units of $\mathrm{cm} / \mathrm{s}$ is obtained. This rate constant appears to be the most appropriate for comparing one waste form wi h another. These comparisons show that silicateagglomerated $\mathrm{Fe}_{2} \mathrm{O}_{3}$ (a stand-in for NFS sludge material) has the lowest rate of dissolution in quiescent water.

Table 1. Dissolution/Disintegration Rate Constants $\mathbf{a}^{\mathrm{a}}$

\begin{tabular}{|c|c|c|c|}
\hline Materials & $\begin{array}{l}\text { Density, } \\
\mathrm{g} / \mathrm{cm}^{3}\end{array}$ & $\begin{array}{l}\text { Rate } \\
\text { Constant, } \\
g / \mathrm{cm}^{2}-\mathrm{s}\end{array}$ & $\begin{array}{l}\text { Rate } \\
\text { Constant, } \\
\mathrm{cm} / \mathrm{s}\end{array}$ \\
\hline Salt Block & 2.14 & $4.8 \times 10^{-4}$ & $2.2 \times 10^{-4}$ \\
\hline $\begin{array}{l}\text { NFS Waste Salt } \\
\text { (Supernate with Sludge) }\end{array}$ & 2.24 & $9.7 \times 10^{-4}$ & $4.3 \times 10^{-4}$ \\
\hline (Supernate Only) & 2.22 & $9.7 \times 10^{-4}$ & $4.4 \times 10^{-4}$ \\
\hline $\begin{array}{l}\text { Brach's Sparkler } \\
\text { (lemon) }\end{array}$ & 1.53 & $3.1 \times 10^{-4}$ & $2.0 \times 10^{-4}$ \\
\hline $\begin{array}{l}\text { Sodium Silicate } \\
\text { SS-C/ } \mathrm{Fe}_{2} \mathrm{O}_{3} \text { Pellet } \\
6 / 1\end{array}$ & 2.48 & $1.6 \times 10^{-5}$ & $6.4 \times 10^{-6}$ \\
\hline $\begin{array}{l}\text { Sodium Silicate } \\
\text { SS-C/Fe } 2 \mathrm{O}_{3} \text { Pellet } \\
2 / 1\end{array}$ & 2.48 & $5.3 \times 10^{-5}$ & $2.1 \times 10^{-5}$ \\
\hline $\begin{array}{l}\text { Phosphate-Imnobilized } \\
\text { Exp. 209-140 }\end{array}$ & 2.64 & $2.2 \times 10^{-4}$ & $8.5 \times 10^{-5}$ \\
\hline $\begin{array}{l}\text { Phosphate-Immobilized } \\
\text { Exp. 209-147 }\end{array}$ & 1.68 & $9.4 \times 10^{-5}$ & $5.6 \times 10^{-5}$ \\
\hline $\begin{array}{l}\text { Phosphate-Immobilized } \\
\text { Exp. 214-03 }\end{array}$ & 2.30 & $2.0 \times 10^{-4}$ & $8.7 \times 10^{-5}$ \\
\hline $\begin{array}{l}\text { Phos phate-Immotilized } \\
\text { Exp. 214-08 }\end{array}$ & 2.50 & $3.3 \times 10^{-4}$ & $1.3 \times 10^{-4}$ \\
\hline
\end{tabular}

\footnotetext{
${ }^{a} \mathrm{~A} 1 \mathrm{l}$ measurements were made at ambient temperature $\left(20-26^{\circ} \mathrm{C}\right)$ in $4000 \mathrm{~mL}$ of stagnant deionized water.

${ }^{b}$ The compositions of these materials are described in the text.
} 
The dissolution/disintegration rates of phosphateimmobilized simulated NFS wastes are disappointing. The rate, fivefold lower than that obtained by merely dehydrating the $\mathrm{NaNO}_{3} / \mathrm{NaNO}_{2}$ salt solution, may not be worth the extra difficulty in preparing this form. It is important to note, however, that although the solid product from these distillation experiments appeared to be completely homogeneous and appeared to disintegrate homogeneously, at the end of disintegration, the product was nonhomogeneous-a colorless leachate and a very fine white powder remained in the beaker. Samples of the leachate from phosphate-immobilized samples were analyzed for metal cation content by $d-c$ arc spectrometry. These analyses show that the only cation soluble in the leachate of these interim waste forms is sodium ion. Iron, for example, was determined to be present in the leachate at less than $0.1 \%$ of the concentration possible if the iron had all dissolved.

There were only trivial differences in dissolution/disintegration rates for the four phosphate-immobilized waste forms studied. The dissolution/disintegration rate was not significantly altered by changing the volume ratio of $\mathrm{H}_{3} \mathrm{PO}_{4}$ to simulated NFS waste in the narrow range studied.

The same penetration rate was measured for simulated NFS fused salt, whether the sludge components were present or absent. This result would be expected since the sludge material is only 8 wt $\%$ of the fused salt and there is no significant chemical reaction possible between the supernate and the sludge components in the solid state.

\section{c. Thermal Properties of Phosphate-Immobilized Simulated NFS Waste}

A preliminary differential thermal analysis (DTA) in the range 20-1000 $\mathrm{C}$ was run on phosphate-immbilized simulated NFS waste prepared in Exp. 209-140. There were three distinct endothermic regions. The first occurred at about $275^{\circ} \mathrm{C}$; the second, a wide region with many changes occurring one after another, began at about $570^{\circ} \mathrm{C}$; the third was in the region of $950-$ $1000^{\circ} \mathrm{C}$. Thermal gravimetric analyses will be performed on this same material to learn whether these changes were due to melting, a chemical reaction, or vaporization of constituents. Discussion of the significance of this DTA has been postponed until more experimental iata are obtained. The same kind of experiments will also be performed for other preparations of phosphateimmoilized simulated NFS waste.

\section{Stability of $\mathrm{FePO}_{4}$ (G. F. Vandegrift and E. T. Kucera ${ }^{\star}$ )}

Because there is a question as to whether large amounts of $\mathrm{FePO}_{4}$ are stable in Nuclear Fuel Services (NFS) sludge [STEINDLER-1981C], the following reaction is being studied:

$$
2 \mathrm{FePO}_{4(8)}+(\mathrm{n}+3)_{2} \mathrm{H}_{2} \mathrm{O}=\mathrm{Fe}_{2} \mathrm{O}_{3} \cdot \mathrm{nH}_{2} \mathrm{O}(\mathrm{B})+2 \mathrm{H}_{3} \mathrm{PO}_{4}
$$

\footnotetext{
${ }^{\star}$ Member of the Analytical Chemistry Laboratory, Chemical Engineering Division.
} 
Experiments are beirg performed to determine the extent of reaction of FePO $4 \cdot 2 \mathrm{H}_{2} \mathrm{O}$ solid with an aqueous solution simulat ing NFS supernate $(6 \mathrm{M} \mathrm{NaNO}$ and $0.18 \mathrm{M} \mathrm{NaOH})$. The reaction is being investigated by measuring the concentrations of iron and phosphorus in the aqueous phase contacted with various amounts of $\mathrm{FePO}_{4} \cdot 2 \mathrm{H}_{2} \mathrm{O}(+90 \mu \mathrm{m}-180 \mu \mathrm{m}$ particle size) for various times. The ratio of iron to phosphorus in the solid residues of these reactions is also being measured.

The technique used for each sample to measure iron and phosphorus simul$t$ aneously is energy-dispersive X-ray fluorescence (EDXRF). A sample is prepared for analysis by EDXRF by evaporsting to dryness aqueous solutions and drying all residues overnight in an $85^{\circ} \mathrm{C}$ oven, combining the dried residue with cellulose binder ( 3 parts dried residue to 1 part cellulose), and pressing the mixture into a $3 / 4-$ in.-dia $1-g$ pellet.

At this time, only qualitative statements can be made about the hydrolys is react ion:

1. The higher the $\mathrm{pH}$, the further reaction 3 proceeds and the more rapid is its approach to equilibrium.

$$
2 \mathrm{FePO}_{4}+6 \mathrm{OH}^{-}+\mathrm{mH}_{2} \mathrm{O} \rightleftharpoons \mathrm{Fe}_{2} \mathrm{O}_{3} \cdot \mathrm{mH}_{2} \mathrm{O}(\mathrm{s})+2 \mathrm{PO}_{4(\mathrm{aq})}^{3-}
$$

2. The extent to which the above reaction progresses to the right is limited by the solubility of $\mathrm{PO}_{4}^{3-}$ in the $\mathrm{NaNO}_{3}$ solution and possibly by a surface reaction of $\mathrm{PO}_{4}^{3-}$ with solid $\mathrm{Fe}_{2} \mathrm{O}_{3}$ or $\mathrm{FePO}_{4}$.

3. If the composition of the supernate in Tank $8 D 2$ is as reported in the companion report [WNYNSCS], then, based on the experiments reported above, $\mathrm{FePO}_{4}$ should be absent from the sludge; all ferric iron siould be present as $\mathrm{Fe}_{2} \mathrm{O}_{3}$.

These results are preliminary and will be discussed further when more data are available.

D. Interim Waste Form Development: Materials Studies

(G. Bandyopadhyay, ${ }^{\star}$ J. E. Slattery, ${ }^{\star}$ and T. M. Galvin ${ }^{\star}$ )

The work performed during the past quarter includes (1) completion of the studies of denaification, fracture behavior, and stability in the presence of water of several sintered silicate simulated sludge ${ }^{\dagger}$ specimens and (2) continuation of the investigation of the properties of specimens containing sodium silicate/calcium hydroxide binder. The results are summarized below.

\footnotetext{
* Member of the Materials Science Division, Argonne National Laboratory. ${ }^{\dagger}$ Simulat ing Nuclear Fuel Services ludge.
} 


\section{Sintered Silicate/Sludge System}

Several silicate/sludge specimens containing Ss-65 $\left(\mathrm{SiO}_{2}: \mathrm{Na}_{2} \mathrm{O}=\right.$ $3.22: 1.00)$ or $\mathrm{SS}-\mathrm{C}\left(\mathrm{SiO}_{2}: \mathrm{Na}_{2} \mathrm{O}=2.00: 1.00\right)$ sodium silicate ${ }^{\star}$ and 30,50 , or 70 wt \% synthetic sludge were cold-pressed at different compacting pressures (from 28 to $351 \mathrm{MPa}$ ) and subsequent ly fired at $600^{\circ} \mathrm{C}$ for $4 \mathrm{~h}$. Figure 4 shows the sintered density of these specimens as a function of sludge content and compacting pressure. The strength data of these specimens, as obtained by the diametral compression technique, are shown in Fig. 5. It is evident that both SS -65 and SS-C compacts containing 250 wt $\%$ sludge were mechanically extremely weak (strength $0-0.5 \mathrm{MPa}$ ). High compacting pressure improved the strength to some extent. However, only SS-C compacts containing $30 \mathrm{wt} \%$ sludge and that had been compacted at $2211 \mathrm{MPa}$ exhibited the desired mechanical strength.

In contrast to the data presented in Fig. 5, recent experiments with sludge/silicate specimens containing 75 wt \% synthetic sludge and 25 wt \% SS -65 or SS-C indicated that substantially stronger pellets (fracture strength, about $10 \mathrm{MPa}$ ) could be prepared by exposing the specimens to a high-humidity environment at $35^{\circ} \mathrm{C}$ prior to the sintering step. Additional tests are being performed to confirm this finding. The mechanisms contributing to the improvement of the mechanical properties of the pellets are also being investigated.

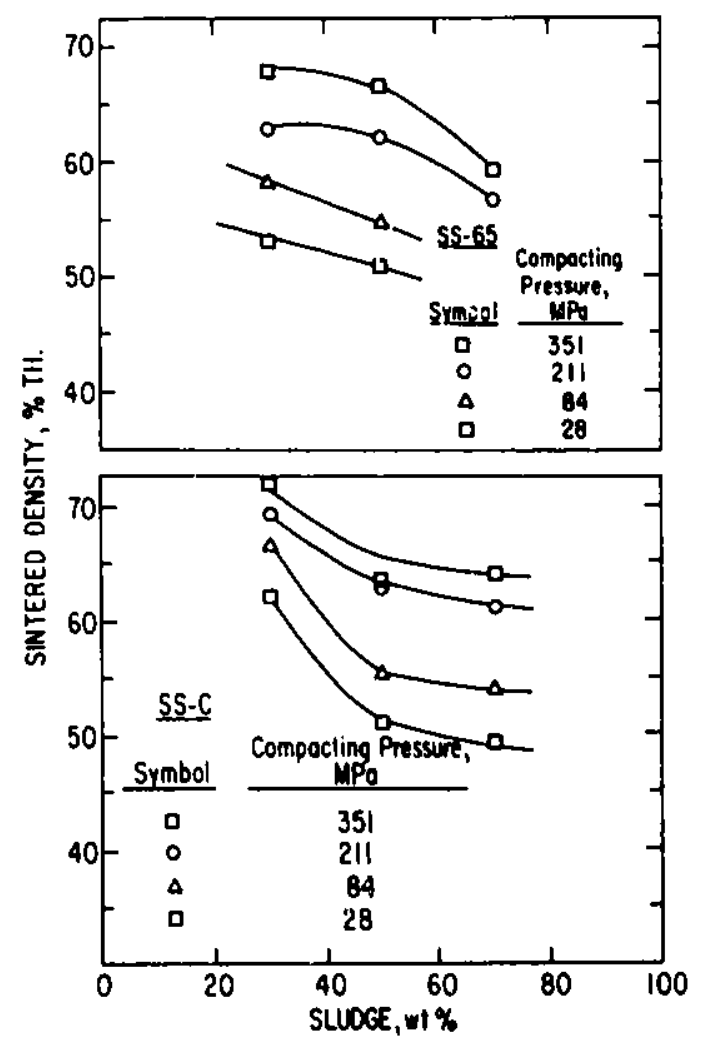

Fig. 4.

Sintered Density of SS-65 and SS-C Specimens as a Function of Compacting Pressure during Fabrication and Sludge Content of the Specimens

* Sodium silicates were obtained from PQ Corporation, Valley Forge, PA. 


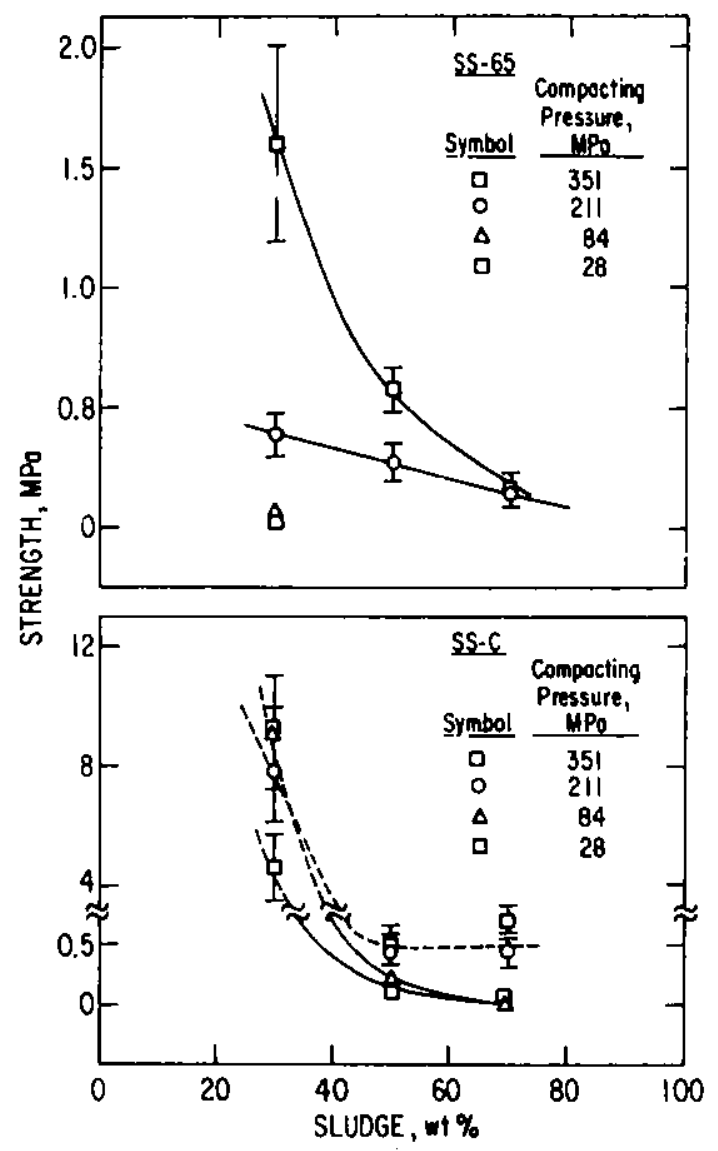

Fig. 5 .

Fracture Strength, as Measured by Diametral Compression Technique, of Sintered SS-65 and SS-C Specimens as a Function of Compacting Pressure during Fabrication and Sludge Content of the Specimens

The stability in water of silicate/sludge specimens (prepared by conventional cold-pressing and sintering technique without the humidity treatment) was examined by determining the time taken for collapse of the specimens when immersed in $4000 \mathrm{~cm}^{3}$ of static water at room temperature. Both sS-C and SS-65 sintered cylindrical compacts (surface area, about $5 \mathrm{~cm}^{2}$ ) containing 30 wt \% sludge and cold-pressed at 351-MPa pressure collapsed in about 2-3 $\mathrm{h}$. The remaining specimens, containing $>30$ wt $\%$ sludge and/or cold-pressed at lower pressures, failed in shorter time periods. Although pure SS -65 powder is one-sixth as water-soluble as pure SS-C powder at $25^{\circ} \mathrm{C}$, the silicate/s ludge specimens did not show any strong effect of composition on the solubility. $\mathrm{ss}-\mathrm{C} / \mathrm{sludge}$ compacts were, in fact, somewhat more stable in water than similar SS-65/sludge specimens-probably because of the superior mechanical stability of the former compositions.

The silicate/sludge specimens were, in general, considerably more resiatant to water than were fused-salt samples simulating the salt ratio in the NFS supernatant composition. A fused-salt specimen of surface area about $5 \mathrm{~cm}^{2}$ and containing 20 wt $\%$ sludge dissolved in about 30-40 min. Additional water stability tests will be performed on silicate/sludge opecimens prepared by the improved processing technique (which includes the humidity treatment prior to the sintering tep). 


\section{Cement-Silicete Systew}

We reported earlier [STEINDLER-1981C] that a potential interim waste form having attractive chemical and mechanical properties was prepared in the laboratory by a room-temperature process, using sodium silicate and calcium hydroxide as a binder. Reaction between sodium silicate and calcium hydroxide in the presence of moisture forms a cementitious bond which provides the desirable chemical and mechanical properties of the waste form. Extensive studies have been initiated during this reporting period to identify optimal compositions of the sodium silicate-calcium hydroxide mixture for achieving desirable properties at a reasonably high sludge loading. Some of the data are presented below.

A series of powder mixtures was prepared by intimately blending various proportions of synthetic sludge (simulating NFS waste) with anhydrous, amorphous sodium silicate (SS-65 and SS-C compositions) and calcium hydroxide. Cylindrical specimens were cold-pressed, and samples were then exposed to a controlled high-humidity air ( $90 \%$ relative humidity) for $24 \mathrm{~h}$ at $35^{\circ} \mathrm{C}$. Determined were the weight gain of the samples due to water absorption, the mechanical strength, and the room temperature stability of the specimens in static water. Table 2 summarizes the water absorption and fracture strength data for various compositions.

Table 2. Water Absorption during the Humidity Treatment; the Fracture Strength of Specimens Containing Synthetic Sludge, Sodium Silicate, and $\mathrm{Ca}(\mathrm{OH})_{2}$

\begin{tabular}{|c|c|c|c|c|}
\hline \multirow[b]{2}{*}{$\begin{array}{l}\text { Sludge, } \\
\text { wt } \%\end{array}$} & \multicolumn{2}{|c|}{ Composition } & \multirow[b]{2}{*}{$\begin{array}{c}\text { Water } \\
\text { Absorption, } \\
\text { wt } \%\end{array}$} & \multirow[b]{2}{*}{$\begin{array}{l}\text { Fracture } \\
\text { Strength, a } \\
\text { MPa }\end{array}$} \\
\hline & $\begin{array}{l}\text { Sodium } \\
\text { Silicate, } \\
\text { wt } \%\end{array}$ & $\begin{array}{c}\mathrm{Ca}(\mathrm{OH})_{2} \\
\text { wt } \%\end{array}$ & & \\
\hline \multicolumn{5}{|l|}{ ss-65 } \\
\hline $\begin{array}{l}50.0 \\
90.0 \\
75.0 \\
75.0 \\
75.0 \\
75.0\end{array}$ & $\begin{array}{r}25.0 \\
5.0 \\
25.0 \\
20.0 \\
12.5 \\
0\end{array}$ & $\begin{array}{r}25.0 \\
5.0 \\
0 \\
5.0 \\
12.5 \\
25.0\end{array}$ & $\begin{array}{l}8.91 \\
2.42 \\
6.19 \\
6.90 \\
3.70 \\
2.33\end{array}$ & $\begin{array}{l}7.2 \\
2.3 \\
4.0 \\
7.2 \\
4.7 \\
2.4\end{array}$ \\
\hline \multicolumn{5}{|l|}{$\underline{\mathrm{sS}-\mathrm{C}}$} \\
\hline $\begin{array}{l}75.0 \\
75.0 \\
75.0\end{array}$ & $\begin{array}{l}25.0 \\
20.0 \\
12.5\end{array}$ & $\begin{array}{r}0 \\
5.0 \\
12.5\end{array}$ & $\begin{array}{c}17.62 \\
11.63 \\
6.5\end{array}$ & $\begin{array}{l}7.3 \\
4.2\end{array}$ \\
\hline
\end{tabular}

${ }^{a}$ Standard deviation, $\pm 20 \%$. 
Note that pellets of sufficient strength were obtained with compositions containing 75 wt $\%$ sludge, 20 wt \% sodium silicate, and 5 wt $\% \mathrm{Ca}(\mathrm{OH})_{2}$. Immersion of the specimens containing silicates and $\mathrm{Ca}(\mathrm{OH})_{2}$ in static water at room temperature for $24 \mathrm{~h}$ indicated no detectable weight $108 \mathrm{~s}$, whereas the specimens containing only sodium silicate collapsed in water during similar treatment. Additional experiments are continuing on the chemical and mechanical stability of the pellets during high-temperature (about $600^{\circ} \mathrm{C}$ ) treatment ( $s$ imulating hot fire condition), the impact properties, and the effect of small amounts of supernatant salt on the properties of the cement-silicate waste form. 
II. NEUTRON ACTIVATION AND TRACER STUDIES

(L. J. Jardine, J. K. Bates, and $\mathrm{J}$. E. Kincinas)

\section{A. Introduction}

Dispersion of wastes into the biosphere is the principal potential hazard of nuclear waste disposal. Characterization of waste forms requires the use of sensitive analytical methods to obtain data on low-rate dispersion. Neutron activation analys is (NAA) has been shown to be one applicable method for measuring leach rates of simulated waste forms. Radioactive tracers (the spiked leach method) can provide sensitive methods for applications in which NAA is not possible or practical. Both methods are likely to be useful for characterization measuremenis other than leach rates.

The general objective of this program is to develop techniques and to qualify methods that utilize neutron activation analys is and radioactive tracers for characterizing simulated waste forms. By comparison of characterization test results obtained using these two methods with other existing characterization data, conditions can be specified under which such tests and the resulting data may be extrapolated to fully radioactive specimens. The current focus is on leach-rate characterization of simulated waste glasses and, to a lesser extent, advanced waste forms.

Major goals are: (1) completion of the development of the NAA method for leach-rate determinations, (2) definition of the accuracy, sensitivity, and limitations of NAA, and (3) establishment of the applicability of the test results to larger-scale waste forms. Goals for the development of methods utilizing radioactive tracers are similar.

\section{B. Qualification of Radioactive Tracer Method}

\section{Introduction}

A series of experiments have been defined to qualify the use of NAA and radioactive tracers to measure the leach rates or SRL frit 131 and SRL frit 211 waste glasses. Four classes of chemical elements, present as minor ( $<5$ wt \%) element $s$ in SPL defense wastes and of concern to radioactive waste management, will be the focus of the measurements. Later, PNL 76-68 glass may also be used. The four classes are alkali metals (Cs), alkaline earths ( $\mathrm{Sr}$ and $\mathrm{Ba}$ ), rare earths ( $\mathrm{Ce}$ and $\mathrm{Eu}$ ), and noble metals ( $\mathrm{Ru}$ ). Samples of simulated waste glass spiked with radioactive tracers have been prepared; this glass is being leached directly; also, spiked and nonspiked glasses are submitted for neutron activation prior to leaching.

Leach rates will be measured wherever possible by four different techniques: (1) conventional chemical solution analysis (ICP, ${ }^{*} \mathrm{AA},{ }^{\dagger} \mathrm{DCP}, \emptyset$ flame emission) of leachates from nonneutron-activated glass, (2) gamma-ray spectroscopic analysis of leachate solutions from neutron-activated glass

\footnotetext{
* Ion-coupled plasma emission spectroscopy.

${ }^{\dagger}$ At omic absorption.

$\neq$

Direct current plasma emission spect roscopy.
} 
specimens (A), (3) gamma-ray spectroscopic analysis of leachate solutions from glass specimens containing radioactive tracers (S), (4) gamma-ray spectroscopic analysis of leachate solutions from neutron-activated glass specimens containing radioact ive tracers (S/A).*

The objective of these measurements is to obtain comparative leach rate data in order to define the reproducibility, sensitivity, limitations, and precision of leach rate measurements by the various methods.

\section{Radioact ive Glass Preparation and Source Count ing}

Gamma-ray analyses of the current series of samples have been done by $R$. Malewicki of the Analytical Chemistry Laboratory (ACL), Argonne National Laboratory; by the University of Missouri ( $U$ of $M$ ); and by the Fuel Cycle Section (FCS) of the Chemical Engineering Division (CEN). To ensure that the results from each laboratory are consistent, careful documentation and calibration of each procedure has been done. For the FCS/CEN procedure, calibration over the energy range of $100-2000 \mathrm{keV}$ of the absolute photopeak efficiency of the Ge gamma-ray spectroneter has been performed during this period. Calibrations were done at $5,10,20,30$, and $40 \mathrm{~cm}$ source-to-detector dist ances. St and ards included $182 \mathrm{Ta}, 241_{\mathrm{Am}}, 57_{\mathrm{Co}}, 133_{\mathrm{Ba}} 22_{\mathrm{Na}}, 137 \mathrm{Cs}, 54 \mathrm{Mn}$, $88 \mathrm{Y}, 60^{\circ o}$, and $152 \mathrm{Eu}$. A FORTRAN computer program was written to calculate the absolute photopeak efficiencies. Preliminary results are shown in Fig. 6 .

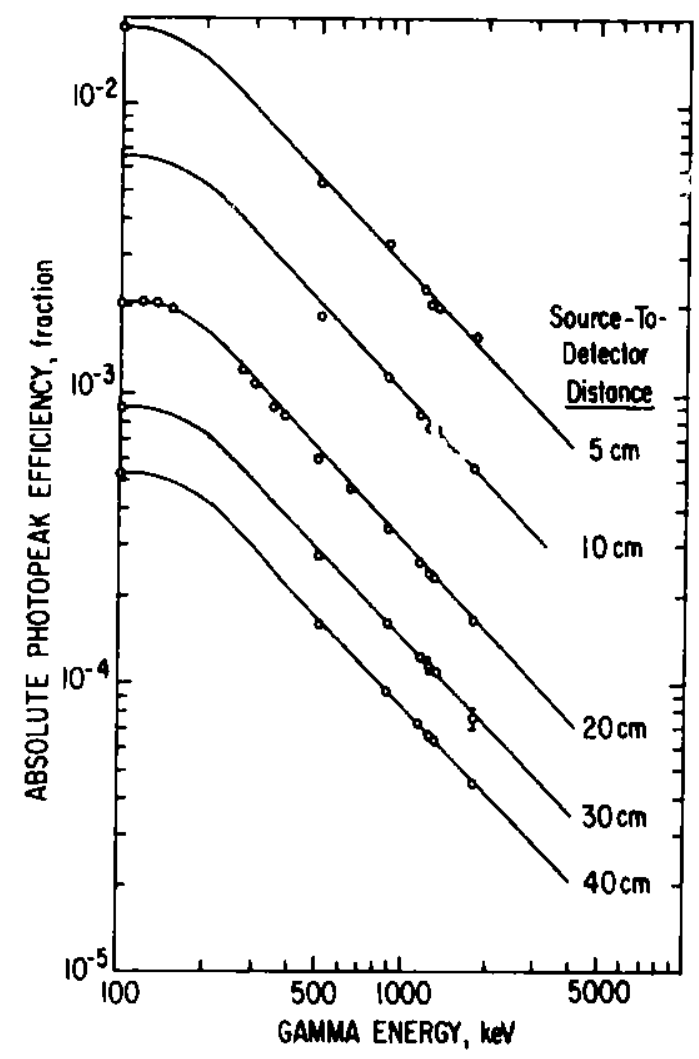

Fig. 6 .

Measured Absolute Photopeak Efficiencies of Germanium Detector as a Function of Gamma Energy and Source-toDetector Distances for St andard Given in this Text

\footnotetext{
" "S" represents spiked glass. "S/A" represents spiked and activated glass. "A" represents activated glass.
} 
Several data points are being remeasured because of the high count rates and poor peak shapes at the closer distances with the available standards. A procedure is being developed for the analysis of high-priority samples of leachates and solids on the Ge gamma-ray spectrometer system.

No new preparations were made during the report period, but more samples of already-cast SRL $211^{\star \star \star}(\mathrm{S})$ and SRL $131^{\star}$ (S) glass we re sectioned and prepared for leach testing. The tests will be analyzed using the FCS/CEN detector calibrated with the above-mentioned standards.

\section{Leaching Characterizations}

To qualify the spike/leach method, tests are being done on five different glasses, SRL-211*, SRL $211^{\star \star}$, SRL $211^{\star \star \star}$, SRL 131 , and SRL $131^{\star}$ (compositions, previously reported, are presented again in Table 3 ). For each type

Table 3. Nominal Compositions of Simulated SRL Waste Glasses Used in this Work

\begin{tabular}{|c|c|c|c|c|c|c|}
\hline & \multicolumn{6}{|c|}{ Wt $\%$} \\
\hline & $\begin{array}{l}\text { SRL } \\
211^{a}\end{array}$ & $\begin{array}{l}\text { SRL } \\
211 *\end{array}$ & $\begin{array}{c}\text { SRL } \\
211 * *\end{array}$ & $\begin{array}{c}\text { SRL } \\
211 * \star \star\end{array}$ & $\begin{array}{l}\mathrm{SRL} \\
131^{\mathrm{b}}\end{array}$ & $\begin{array}{l}\text { SRL } \\
131 *\end{array}$ \\
\hline \multicolumn{7}{|c|}{ Element } \\
\hline $\mathrm{Si}$ & 20.34 & 20 & 19.65 & 19.60 & 19.9 & 19.4 \\
\hline $\mathrm{Na}$ & 11.5 & 11.15 & 11.04 & 11.08 & 9.9 & 9.7 \\
\hline $\mathrm{Fe}$ & 10.28 & 10.08 & 9.75 & 9.91 & 9.7 & 9.5 \\
\hline B & 2.48 & 2.43 & 2.40 & 2.39 & 3.3 & 3.2 \\
\hline $\mathrm{Ca}$ & 3.78 & 3.78 & 3.66 & 3.64 & 0.9 & 0.9 \\
\hline Mn & 2.46 & 2.41 & 2.38 & 2.37 & 2.3 & 2.2 \\
\hline $\mathrm{Al}$ & 2.01 & 1.96 & 1.94 & 1.94 & 1.8 & 1.7 \\
\hline $\mathrm{Li}$ & 1.48 & 1.47 & 1.44 & 1.44 & 1.9 & 1.8 \\
\hline $\mathrm{Ni}$ & 1.34 & 1.31 & 1.29 & 1.29 & 1.3 & 1.3 \\
\hline $\mathrm{Mg}$ & - & - & - & - & 0.9 & 0.9 \\
\hline $\mathrm{Ti}$ & - & - & - & - & 0.4 & 0.4 \\
\hline $\mathrm{Zr}$ & - & - & - & - & 0.3 & 0.3 \\
\hline La & - & - & - & - & 0.3 & 0.3 \\
\hline \multicolumn{7}{|c|}{ Additive } \\
\hline $\mathrm{Cs}$ & - & 0.19 & 0.12 & 0 & - & 0 \\
\hline $\mathrm{Sr}$ & - & 0.07 & 0.30 & 0.32 & - & 0.3 \\
\hline $\mathrm{Ba}$ & - & 0.10 & 0.05 & 0.29 & - & 0.3 \\
\hline $\mathrm{Ce}$ & - & 0.52 & 0.73 & 0.74 & - & 0.7 \\
\hline $\mathrm{Nd}$ & - & 0.63 & 0.79 & 0.81 & - & 0.8 \\
\hline Eu & - & 0.003 & 0.01 & 0.11 & - & 0.1 \\
\hline $\mathrm{Ru}$ & - & - & 0.51 & 0.41 & - & 0.4 \\
\hline $\mathrm{Mg}$ & - & 0.11 & - & 0 & - & 0 \\
\hline $\mathrm{K}$ & - & - & - & 0.05 & - & 0 \\
\hline
\end{tabular}


of glass, the leach rates obtained with spiked glass will be compored to leach rates obtained by another method. The tests described belor are in progress.

a. SRL $211^{\star \star}$

Leach testing of SRL $211^{\star \star}$ glass has been done using cold, activated, spiked, and spiked/activated samples. Leachate analyses and test conditions for each glass type are given in updated Tables 4 and 5 , and weight loss results through 100 days are shown in Fig. 7, a modification of an earlier published figure [STEINDLER-1981C].

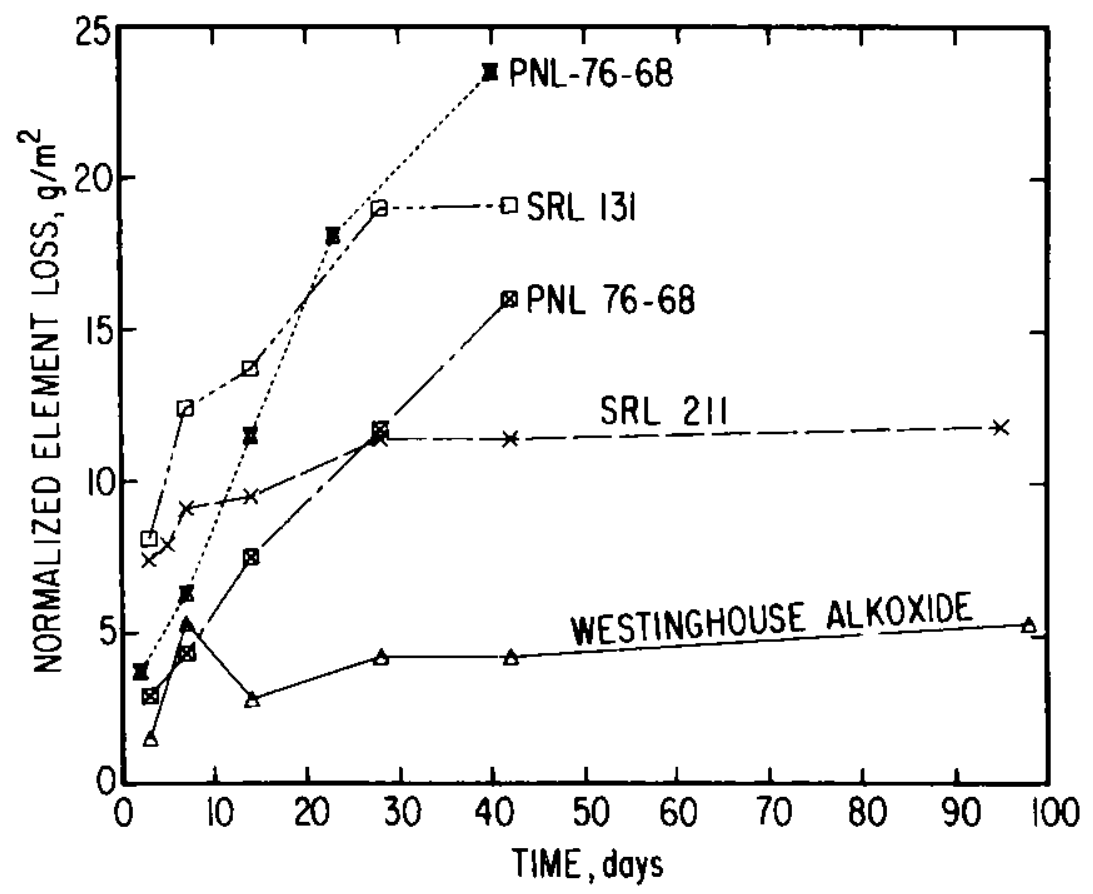

Fig. 7. Summary of Weight LOBs Data for Simulated Waste Glasses

Leaching of activated samples (L-134 to L-146) through 182 days has been completed, and experiments are in progress that will continue for one year. Leachate analysis was done using gamma-ray spectroscopy; for tests through 42.7 days, only $134 \mathrm{Cs}$ could be detected. Since cesium leach rates were being obtained for cold SRL $211^{\star \star}$ glase, no such analyses were planned for activated glass. However, for leachates starting at 98.8 days, both $152 \mathrm{Eu}$ and $141 \mathrm{Ce}$ can also be detected. Thus, complete gamma-ray analyses of samples $\mathrm{L}-143$ (98.8 days) and $\mathrm{L}-144$ (182 days) are in progress.

Cold samples have been leached for 182 days, and an additional test is in progress that will terminate at 364 days. For Cs, $\mathrm{Na}$, and $\mathrm{Li}$, leachate analysis was done using atomic absorption or flame emission by the Analytical Chemistry Laboratory of the Chemical Engineering Division, and for 18 other elements, TEI Analytical used DC plasms sectroscopy. 
Table 4. Summary of Test Conditions, Leachate Analytical Results, and Weight Loss Rates for SRL $211^{\star \star}$ Glass under Study

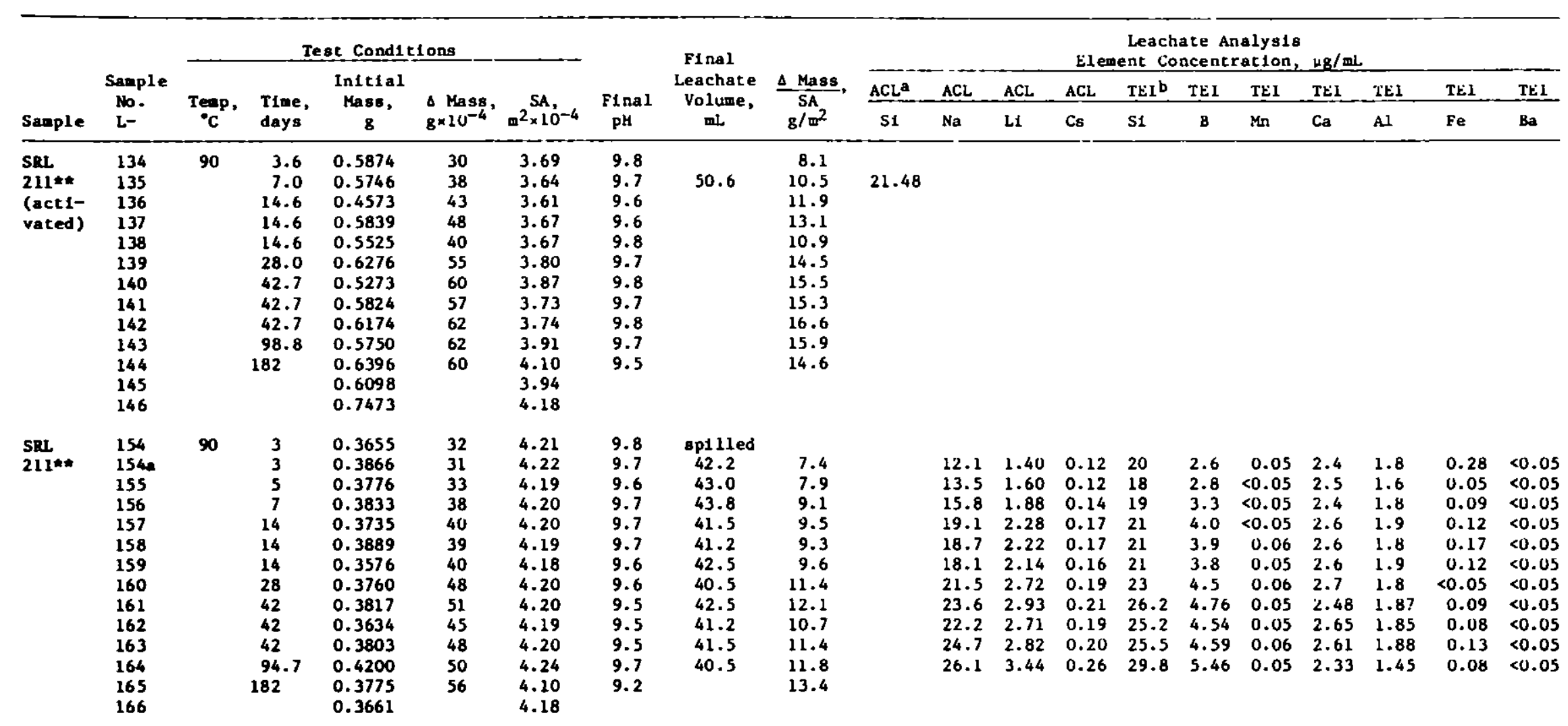


Table 4 (contd)

\begin{tabular}{|c|c|c|c|c|c|c|c|c|c|c|c|c|c|c|c|c|c|c|c|c|}
\hline \multirow{3}{*}{ semple } & \multirow{3}{*}{$\begin{array}{l}\text { Sanple } \\
\text { lo. } \\
\text { L- }\end{array}$} & \multicolumn{5}{|c|}{ Test Conditions } & \multirow{3}{*}{\multicolumn{2}{|c|}{$\begin{array}{c}\text { Final } \\
\text { Leachate } \\
\text { Volume, } \\
\text { mL }\end{array}$}} & \multirow{3}{*}{$\frac{\Delta \text { Mass }}{\mathrm{SA}}$} & \multicolumn{11}{|c|}{$\begin{array}{l}\text { Leachate Analysis } \\
\text { Element Concentration, } \mu g / m \text { m }\end{array}$} \\
\hline & & Tenp, & Tine, & $\begin{array}{c}\text { Initial } \\
\text { Mas, }\end{array}$ & A Mass, & SA, & & & & $\mathrm{ACL}^{\mathrm{a}}$ & ACL & ACL & ACL & TEI ${ }^{b}$ & TEI & TLI & TEI & TEI & TEL & IE I \\
\hline & & ec & day: & & $8 \times 10^{-4}$ & $m^{2} \times 10^{-4}$ & & & & Si & $\mathrm{Na}$ & Li & Cs & $\mathbf{S i}$ & B & $M n$ & $\mathbf{C a}$ & Al & $\mathbf{F e}$ & Ba \\
\hline $\begin{array}{l}\text { sel } \\
211^{\text {*k }} \\
\text { (aplked } \\
\text { and } \\
\text { act1- } \\
\text { vated) }\end{array}$ & $\begin{array}{l}222 \\
223 \\
224 \\
225 \\
226 \\
227 \\
228 \\
229 \\
230 \\
231\end{array}$ & 90 & $\begin{array}{r}3 \\
7 \\
14 \\
14 \\
14 \\
28 \\
42 \\
42 \\
42 \\
95\end{array}$ & $\begin{array}{l}0.4393 \\
0.4505 \\
0.4577 \\
0.4537 \\
0.4528 \\
0.4532 \\
0.4542 \\
0.4504 \\
0.4572 \\
0.4526\end{array}$ & $\begin{array}{l}23 \\
35 \\
42 \\
46 \\
42 \\
\\
46 \\
49 \\
47 \\
47\end{array}$ & $\begin{array}{l}3.90 \\
3.93 \\
3.93 \\
3.86 \\
3.91 \\
3.97 \\
3.95 \\
3.95 \\
3.97 \\
3.97\end{array}$ & $\begin{array}{l}9.5 \\
9.7 \\
9.4 \\
9.4 \\
9.4 \\
9.3 \\
9.7 \\
9.5 \\
9.7 \\
9.7\end{array}$ & $\begin{array}{l}39.7 \\
38.0 \\
37.5 \\
37.9 \\
38.1 \\
35.3 \\
32.3 \\
32.4 \\
33.0 \\
23.2\end{array}$ & $\begin{array}{r}5.9 \\
8.9 \\
10.7 \\
11.2 \\
10.7 \\
\\
11.7 \\
12.4 \\
11.8 \\
11.8\end{array}$ & $\begin{array}{l}35.5 \\
35.4 \\
34.4 \\
37.2\end{array}$ & & & & & & & & & & \\
\hline $\begin{array}{l}5 \text { DI. } \\
211 \text { \#\# } \\
\text { (opiked) }\end{array}$ & $\begin{array}{l}257 \\
258 \\
259 \\
260 \\
261 \\
262 \\
263 \\
264 \\
265 \\
266\end{array}$ & 90 & $\begin{array}{l}3 \\
7 \\
14 \\
14 \\
14 \\
28 \\
42 \\
42 \\
42 \\
95\end{array}$ & $\begin{array}{l}0.4194 \\
0.4549 \\
0.4297 \\
0.4152 \\
0.4493 \\
0.5513 \\
0.4597 \\
0.4432 \\
0.4483 \\
0.4424\end{array}$ & $\begin{array}{l}25 \\
38 \\
44 \\
44 \\
41 \\
45 \\
44 \\
47 \\
42\end{array}$ & $\begin{array}{l}3.91 \\
3.74 \\
3.94 \\
3.99 \\
\\
3.98 \\
3.98 \\
3.92 \\
3.89\end{array}$ & $\begin{array}{l}9.5 \\
9.5 \\
9.4 \\
9.4 \\
9.4 \\
9.4 \\
9.6 \\
9.6 \\
9.4 \\
9.6\end{array}$ & $\begin{array}{l}39.0 \\
38.9 \\
37.5 \\
37.0 \\
37.9 \\
35.8 \\
32.6 \\
33.1 \\
32.8 \\
25.6\end{array}$ & $\begin{array}{r}9.7 \\
11.8 \\
11.2 \\
10.3 \\
11.3 \\
11.1 \\
12.0 \\
10.8\end{array}$ & $\begin{array}{l}36.2 \\
35.3 \\
37.2 \\
51.4\end{array}$ & & & & & & & & & & \\
\hline
\end{tabular}

\section{(contd)}


Table 4. (contd)

\begin{tabular}{|c|c|c|c|c|c|c|c|c|c|c|c|}
\hline \multirow[b]{3}{*}{ Smple } & \multirow{3}{*}{$\begin{array}{l}\text { Semple } \\
\text { Ro. } \\
\text { L- }\end{array}$} & \multicolumn{10}{|c|}{ Element Concentration $\left(A_{i} / \Lambda_{0} \times 10^{3}\right)$} \\
\hline & & \multicolumn{10}{|c|}{ NAM by either ACL or University of Missouri } \\
\hline & & ${ }^{51} \mathrm{cr}$ & ${ }^{59} \mathrm{Fe}$ & ${ }^{85} S_{r}$ & ${ }^{124} \mathrm{sb}$ & ${ }^{131} 1_{\mathrm{Ba}}$ & ${ }^{134} \mathrm{Cs}$ & ${ }^{137} \mathrm{CB}$ & ${ }^{141} \mathrm{ce}$ & ${ }^{147} \mathrm{Md}$ & ${ }^{152}$ Eu \\
\hline $\begin{array}{l}\text { SRL } \\
\text { 2t1 } \\
\text { (opiked } \\
\text { and } \\
\text { ecti- } \\
\text { vated) }\end{array}$ & $\begin{array}{l}222 \\
223 \\
224 \\
225 \\
226 \\
227 \\
228 \\
229 \\
230 \\
231\end{array}$ & $\begin{array}{l}<.5 \\
2.11 \\
1.55 \\
1.22 \\
2.41 \\
1.77 \\
4.66 \\
3.22\end{array}$ & & & $\begin{array}{l}3.32 \\
4.61 \\
5.04 \\
6.65 \\
6.45 \\
7.04 \\
9.51 \\
8.64 \\
4.41\end{array}$ & $\begin{array}{l}0.31 \\
0.21 \\
0.15 \\
0.22 \\
0.26 \\
0.19 \\
0.16 \\
0.14 \\
0.16\end{array}$ & $\begin{array}{l}4.86 \\
7.55 \\
7.62 \\
8.49 \\
8.62 \\
9.28 \\
12.4 \\
11.2 \\
10.6\end{array}$ & $\begin{array}{c}5.57 \\
8.82 \\
8.87 \\
9.93 \\
10.1 \\
12.0 \\
14.3 \\
13.0 \\
12.4\end{array}$ & $\begin{array}{l}0.025 \\
\text { ND } \\
0.028 \\
0.037 \\
\text { ND } \\
\text { ND } \\
\text { ND } \\
\text { ND } \\
\text { ND }\end{array}$ & & $\begin{array}{l}0.026 \\
\mathrm{ND} \\
\mathrm{ND} \\
0.021 \\
\mathrm{ND} \\
0.033 \\
\mathrm{ND} \\
0.009 \\
0.010\end{array}$ \\
\hline $\begin{array}{l}\text { spd } \\
\text { 211*t. } \\
\text { (opiked) }\end{array}$ & $\begin{array}{l}257 \\
258 \\
259 \\
260 \\
261 \\
262 \\
263 \\
264 \\
265 \\
266\end{array}$ & & & & & $\begin{array}{l}0.05 \\
0.20 \\
0.19 \\
0.22 \\
0.23 \\
0.14 \\
0.15 \\
0.15 \\
0.14\end{array}$ & & $\begin{array}{r}6.27 \\
7.41 \\
9.69 \\
10.50 \\
9.93 \\
8.82 \\
12.60 \\
12.90 \\
13.40\end{array}$ & & & \\
\hline
\end{tabular}

Analyees doae by the Analytical Chemiatry Laboratory of the Cheaical Engineering Diviaion.

Banlyee done by Trece Elemente, Inc. 
Table 5. Summary of Normalized Leach Rates Calculated from Data

in Table 4 for SRL 211** Glass. Temperature $=90^{\circ} \mathrm{C}$.

\begin{tabular}{|c|c|c|c|c|c|c|c|c|c|c|c|c|c|}
\hline \multicolumn{14}{|c|}{ Normalized Element al Loss, $(\mathrm{NL})_{i}, \mathrm{~g} / \mathrm{m}^{2}$} \\
\hline Saple & $\begin{array}{l}\text { Saple } \\
\text { No. } \\
\text { L- }\end{array}$ & $\begin{array}{l}\text { Time, } \\
\text { days }\end{array}$ & $\begin{array}{c}\mathbf{A C L} \\
\mathbf{S i}\end{array}$ & $\begin{array}{l}\mathrm{ACL} \\
\mathrm{Ha}\end{array}$ & $\begin{array}{l}\text { ACL } \\
\mathbf{L i}\end{array}$ & $\begin{array}{l}\text { ACL } \\
\text { Cs }\end{array}$ & $\begin{array}{l}\text { TE I } \\
\mathbf{S i}\end{array}$ & $\begin{array}{c}\text { TEI } \\
\text { B }\end{array}$ & $\begin{array}{l}\text { TEI } \\
\text { Mn }\end{array}$ & $\begin{array}{l}\text { TEI } \\
\mathrm{Ca}\end{array}$ & $\begin{array}{l}\text { TEI } \\
\mathrm{Al}\end{array}$ & $\begin{array}{l}\text { TEI } \\
\text { Fe }\end{array}$ & $\begin{array}{l}\text { TEI } \\
\text { Ba }\end{array}$ \\
\hline $\begin{array}{l}\text { SRI } \\
211 * \star \\
\text { (acti- } \\
\text { vated) }\end{array}$ & $\begin{array}{l}134 \\
135 \\
136 \\
137 \\
138 \\
139 \\
140 \\
141 \\
142 \\
143 \\
144 \\
145 \\
146\end{array}$ & $\begin{array}{r}3.6 \\
7.0 \\
14.6 \\
14.6 \\
14.6 \\
28.0 \\
42.7 \\
42.7 \\
42.7 \\
98.8\end{array}$ & 15.3 & & & & & & & & & & \\
\hline SRL & 154 & 3 & & & & & & & & & & & \\
\hline $211 * \star$ & $\begin{array}{l}1540 \\
155 \\
156 \\
157 \\
158 \\
159 \\
160 \\
161 \\
162 \\
163 \\
164 \\
165 \\
166\end{array}$ & $\begin{array}{c}3 \\
5 \\
7 \\
14 \\
14 \\
14 \\
28 \\
42 \\
42 \\
42 \\
94.7\end{array}$ & & $\begin{array}{l}11.1 \\
12.8 \\
15.3 \\
17.4 \\
17.0 \\
17.0 \\
19.1 \\
21.8 \\
20.2 \\
22.5 \\
22.7\end{array}$ & $\begin{array}{l}11.2 \\
13.1 \\
15.7 \\
18.0 \\
17.5 \\
17.4 \\
21.0 \\
23.4 \\
21.3 \\
22.3 \\
26.0\end{array}$ & $\begin{array}{l}11.4 \\
11.5 \\
13.7 \\
16.0 \\
16.1 \\
16.1 \\
17.6 \\
20.0 \\
18.1 \\
18.8 \\
23.7\end{array}$ & $\begin{array}{r}10.3 \\
9.5 \\
10.2 \\
10.7 \\
10.6 \\
11.0 \\
11.4 \\
13.5 \\
12.7 \\
12.9 \\
14.6\end{array}$ & $\begin{array}{l}10.7 \\
11.9 \\
14.2 \\
16.3 \\
15.8 \\
16.0 \\
17.9 \\
19.7 \\
18.4 \\
18.7 \\
21.6\end{array}$ & $\begin{array}{r}0.2 \\
<0.2 \\
<0.2 \\
<0.2 \\
0.3 \\
0.2 \\
0.3 \\
0.2 \\
0.2 \\
0.3 \\
0.2\end{array}$ & $\begin{array}{l}7.0 \\
7.5 \\
7.3 \\
7.5 \\
7.5 \\
7.7 \\
7.6 \\
7.2 \\
7.6 \\
7.5 \\
6.5\end{array}$ & $\begin{array}{r}9.9 \\
9.0 \\
10.3 \\
10.3 \\
9.7 \\
10.6 \\
9.5 \\
10.3 \\
10.0 \\
10.2 \\
7.6\end{array}$ & $\begin{array}{l}0.3 \\
0.06 \\
0.1 \\
0.1 \\
0.2 \\
0.2 \\
<0.06 \\
0.1 \\
0.1 \\
0.2 \\
0.1\end{array}$ & \\
\hline
\end{tabular}


Table 5. (contd)

Normalized Elemental Loss, (NL) $i, g / \mathrm{m}^{2}$

\begin{tabular}{|c|c|c|c|c|c|c|c|c|c|c|c|c|c|}
\hline Saple & $\begin{array}{c}\text { Sample } \\
\text { No. } \\
\text { L- }\end{array}$ & $\begin{array}{l}\text { Time, } \\
\text { days }\end{array}$ & $\begin{array}{c}\mathbf{A C L} \\
\mathbf{S i}\end{array}$ & $\begin{array}{l}\text { ACL } \\
\text { Na }\end{array}$ & $\begin{array}{l}\text { ACL } \\
\mathbf{L i}\end{array}$ & $\begin{array}{l}\text { ACL } \\
\mathrm{CB}_{3}\end{array}$ & $\begin{array}{l}\operatorname{TEI}^{b} \\
\mathrm{Si}\end{array}$ & $\begin{array}{c}\text { TEI } \\
\text { B }\end{array}$ & $\begin{array}{l}\text { TEI } \\
\text { Mn }\end{array}$ & $\begin{array}{l}\text { TEI } \\
\mathrm{Ca}\end{array}$ & $\begin{array}{l}\text { TEI } \\
\text { Al }\end{array}$ & $\begin{array}{l}\text { TEI } \\
\text { Fe }\end{array}$ & $\begin{array}{l}\text { TEI } \\
\text { Ba }\end{array}$ \\
\hline $\begin{array}{l}\text { SRL } \\
211 * \hbar \\
\text { (apiked } \\
\text { and } \\
\text { acti- } \\
\text { vated) }\end{array}$ & $\begin{array}{l}222 \\
223 \\
224 \\
225 \\
226 \\
227 \\
228 \\
229 \\
230 \\
231\end{array}$ & $\begin{array}{r}3 \\
7 \\
14 \\
14 \\
14 \\
28 \\
42 \\
42 \\
42 \\
95\end{array}$ & $\begin{array}{l}14.9 \\
14.9 \\
14.7 \\
11.2\end{array}$ & & & & & & & & & & \\
\hline $\begin{array}{l}\text { sa } \\
211 \text { *t } \\
\text { (spiked) }\end{array}$ & $\begin{array}{l}257 \\
258 \\
259 \\
260 \\
261 \\
262 \\
263 \\
264 \\
265 \\
266 \\
234\end{array}$ & $\begin{array}{r}3 \\
7 \\
14 \\
14 \\
14 \\
28 \\
42 \\
42 \\
42 \\
95 \\
182\end{array}$ & $\begin{array}{l}15.2 \\
15.1 \\
16.0 \\
17.4\end{array}$ & & & & & & & & & & \\
\hline
\end{tabular}


Table 5. (contd)

Normalized Elemental Loss, (NL) $i, g / m^{2}$

\begin{tabular}{|c|c|c|c|c|c|c|c|c|c|c|c|c|c|c|c|c|c|}
\hline Sample & $\begin{array}{l}\text { Sample } \\
\text { No. } \\
\text { L- }\end{array}$ & $\begin{array}{l}\text { Time, } \\
\text { day } 8\end{array}$ & ${ }^{51} \mathrm{Cr}$ & ${ }^{59} \mathrm{Fe}$ & ${ }^{85} S_{S r}$ & ${ }^{103} \mathrm{Ru}$ & ${ }^{106} \mathrm{Ru}$ & $131_{\mathrm{Ba}}$ & ${ }^{133_{\mathrm{Ba}}}$ & ${ }^{134} \mathrm{Cs}_{5}$ & ${ }^{137} \mathrm{Cs}$ & ${ }^{139} \mathrm{Ce}$ & ${ }^{141} \mathrm{Ce}$ & $147 \mathrm{Nd}$ & ${ }^{152} \mathrm{Eu}$ & $154 \mathrm{Eu}$ & ${ }^{124} \mathrm{sb}$ \\
\hline $\begin{array}{l}\text { SRL } \\
211^{* t} \\
\text { (acti- } \\
\text { vaced) }\end{array}$ & $\begin{array}{l}134 \\
135 \\
136 \\
137 \\
138 \\
139 \\
140 \\
141 \\
142 \\
143 \\
144 \\
145 \\
146\end{array}$ & $\begin{array}{r}3.6 \\
7.0 \\
14.6 \\
14.6 \\
14.6 \\
28.0 \\
42.7 \\
42.7 \\
42.7 \\
98.8\end{array}$ & & & & & & & & 10.0 & & & 0.36 & & 0.33 & & \\
\hline $\begin{array}{l}\text { SRL } \\
211 \text { *k } \\
\text { (spiked } \\
\text { and } \\
\text { act i- } \\
\text { vated) }\end{array}$ & $\begin{array}{l}222 \\
223 \\
224 \\
225 \\
226 \\
227 \\
228 \\
229 \\
230 \\
231\end{array}$ & $\begin{array}{r}3 \\
7 \\
14 \\
14 \\
14 \\
28 \\
42 \\
42 \\
42 \\
95\end{array}$ & $\begin{array}{l}0.85 \\
3.9 \\
2.9 \\
2.3 \\
4.6 \\
3.1 \\
7.2 \\
5.1 \\
8.0\end{array}$ & & & & & & $\begin{array}{l}0.58 \\
0.39 \\
0.29 \\
0.43 \\
0.49 \\
0.33 \\
0.25 \\
0.22 \\
0.26\end{array}$ & $\begin{array}{r}9.1 \\
14.0 \\
14.2 \\
16.3 \\
16.4 \\
16.0 \\
19.2 \\
17.6 \\
17.0\end{array}$ & $\begin{array}{l}10.4 \\
16.4 \\
16.6 \\
19.0 \\
19.1 \\
20.7 \\
22.3 \\
20.4 \\
19.9\end{array}$ & & $\begin{array}{c}0.05 \\
\text { ND } \\
0.05 \\
0.07 \\
\text { ND } \\
\text { ND } \\
\text { ND } \\
\text { ND } \\
\text { ND }\end{array}$ & & $\begin{array}{c}0.05 \\
\text { ND } \\
\text { ND } \\
0.04 \\
\text { ND } \\
0.06 \\
\text { ND } \\
0.01 \\
0.02\end{array}$ & & $\begin{array}{r}6.2 \\
8.6 \\
9.4 \\
12.7 \\
12.2 \\
12.1 \\
14.8 \\
13.6 \\
7.1\end{array}$ \\
\hline $\begin{array}{l}\text { SRL } \\
211^{\star \star \star} \\
\text { (spiked) }\end{array}$ & $\begin{array}{l}257 \\
258 \\
259 \\
260 \\
261 \\
262 \\
263 \\
264 \\
265 \\
266 \\
234\end{array}$ & $\begin{array}{r}3 \\
7 \\
14 \\
14 \\
14 \\
28 \\
42 \\
42 \\
42 \\
95 \\
182\end{array}$ & & & & & & & $\begin{array}{l}0.92 \\
0.50 \\
0.43 \\
0.46 \\
0.48 \\
0.34 \\
0.28 \\
0.28 \\
0.26 \\
0.44\end{array}$ & & $\begin{array}{l}14.0 \\
18.2 \\
22.8 \\
22.0 \\
20.4 \\
21.9 \\
23.3 \\
23.7 \\
24.3 \\
12.4\end{array}$ & & & & 1.50 & & \\
\hline
\end{tabular}

Analyoes done by the Analytical Chemistry Laboratory of the Chemical Engineering Division.

'Analyses done by Trace Elements, Inc. 
Spiked and spiked/aitivated leach testis were run concurrently to ensure temperature equalization. These 95-day tests have been completed. Gamma-ray analysis for the radionuclides and analysts for ilicon, a matrix element, are nearly complete for the entire series of tests.

Two major 1ssues pertain to the data presented for $211^{\star \star}$ glass: (1) does radiotracer spiking give a reliable measurement of leach rates from simulated nuclear waste glasses and, if it does, what are the optimized tracer loading parameters; (2) what inferences can be $\mathrm{drawn}$ about the leaching behavior of the waste glass? The first point is of the greatest concern to this program and will be developed fully, while the second point, more interesting, will be developed insofar as resources allow.

The followt
ments of SRL $211^{\star \star}$ glass:

(1) The silicon leach rates, (NL) S1 $_{1}$ have been determined for each glass (except activated) at 42 days (Table 4). At this time, triplicate samples were analyzed; within experimental error, no difference in (NL) ${ }_{1} 1$ was observed. (NL) S1 $_{1}$ was also determined at 91 days for the same glasses; the spread of data is larger, but these analyses were for single samples and have a greater uncertainty. Silicon analyses are being done for the 182-day samples, but the results are not available. The data obtained thus far indicate the same rate of leaching of the matrix for all glass treatments.

(2) Cesium leach rates were/are being determined for each type of glass treatment. With only a few analyses remaining to be done, the leach rates, (NL)Cs, measured for cold glass appear to be the same as those for ${ }^{134} \mathrm{Cs}$ measured in S/A glass and $137 \mathrm{Cs}$ measured in S/A and S glass (Table 4).

(3) Barium leach rates could be measured only in S/A and S glass. No barlum could be detected in cold glass using $\mathrm{DC}$ plasma. In S/A and S glass, $133_{\mathrm{Ba}}$ as spiked was detected for all samples and the $(\mathrm{NL})_{\mathrm{Ba}}$ values agreed for the two glasses.

(4) Leach rates for the rare earths, ${ }^{141} \mathrm{Ce}$ and ${ }^{152 \mathrm{Eu} \text {, could }}$ be detected in some of the S/A samples, but the values, $(N L)_{R E}$, are near the detection 11mit. For the $A$ and $S$ samples, Ce and Eu may be detectable in the 91- and 182day samples for which analyses are still in progress.

Overall, for SRL $211^{\text {** }}$ glasses, the leach rates of $\mathrm{Cs}$ have been shown to be consistent for different methods of detection, but the other elements of interest, $\mathrm{Ba}, \mathrm{Ce}, \mathrm{Eu}, \mathrm{Y}$ and $\mathrm{Ru}$ could not be detected for enough glass types (1.e., treatments) to allow comparison of methods of detection. (For this reason, SRL $211^{\star \star}$ was modifled to SRL $211^{\star \star \star}$, which contains less cesium.) 
The general leaching behavior of SRL $211^{\star \star}$ glass is shown in Fig. 8--Na, Li, Cs, B, and $\mathrm{S} 1$ have nearly the same release after three days. All of these elements continue to be leached throughout the next 29 days, but at a reduced rate. The $S i$ rate for this period is significantly less than the rates for the other elements. There is significant release of $\mathrm{Ca}$ and $\mathrm{Al}$ for the first three days, but no release after that. Release of $\mathrm{Mn}$ and $\mathrm{Fe}$

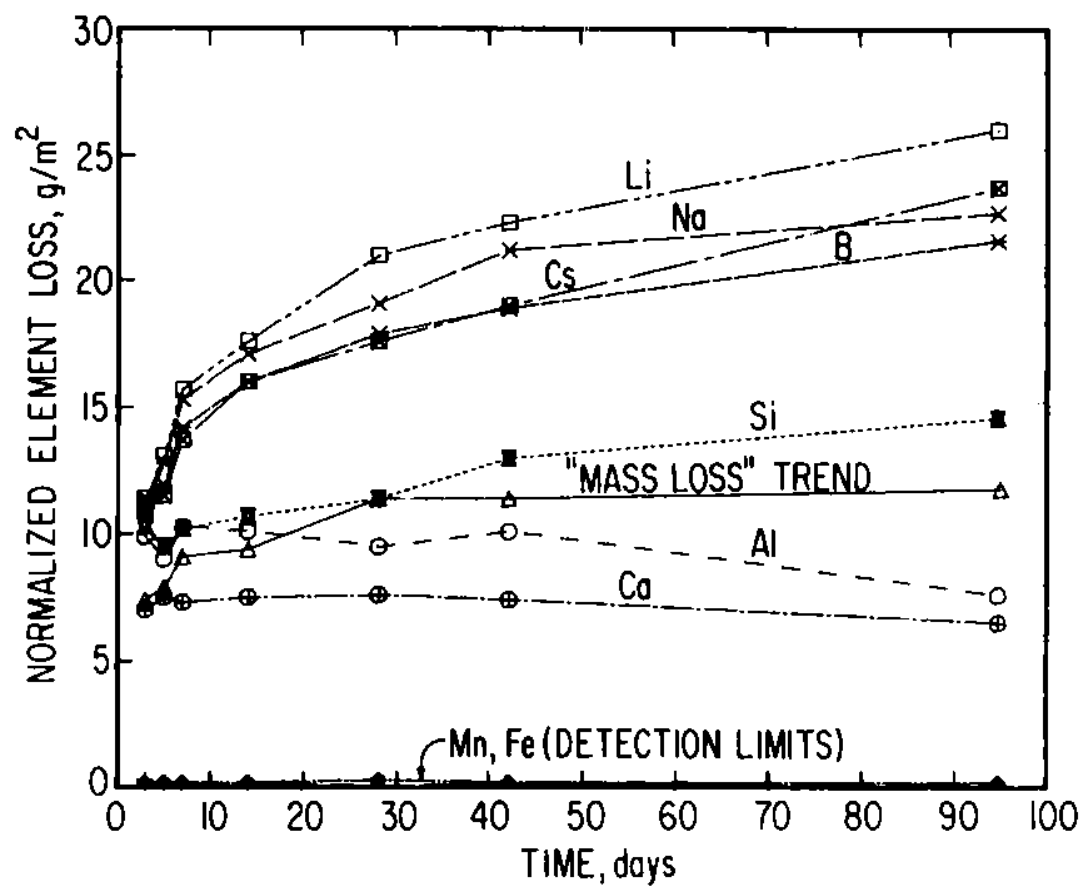

Fig. 8. Preliminary Leach Test Results for Cold SRL $211^{\star \star}$ Glass. MCC-1 tests. Leaching in deionized water at $90^{\circ} \mathrm{C}$.

is initially low and thereafter (NL) $i$ is constant. This leachate analysis data can be combined with surface studies, using SEM/EDAX and electron microprobe analysis. The surface studies indicate that after 91 days of leaching, a reaction layer about $7 \mu \mathrm{m}$ thick exists on the glass. This layer is enriched with $\mathrm{Mn}$ and $\mathrm{Mg}$ at the surface and is somewhat enriched in rare earths throughout. Si and $\mathrm{Na}$ are depleted in the reaction layer.

Surface studies on a sequence of leached samples are in progress in order to wonitor changes occuring with leaching time. The durability of these layers 18 confirmed by long-term weight-loss data which indicate that little additional weight loss occurs after about 20 days of leaching.

b. SRL $211^{\star * *}$

Since SRL $211^{\text {** }}$ glass contalns too much cesium to allow detection of most other gamma-emitters, SRL $211^{\star \star \star}$ glass was made. Th1s glass contains nominally no added cesium. Tests were done on cold, activated, and

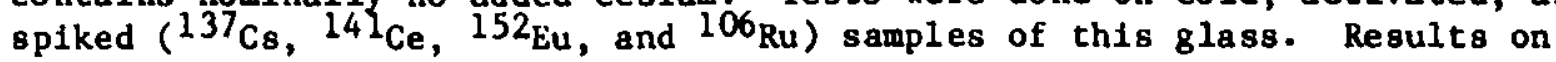


all samples are not complete, but preliminary data (Tables 6 and 7) indicate that the leach rates of all spikes plus more than 10 activated isotopes can be detected. Preliminary results for the activated glass through 28 days are shown in Fig. 9.

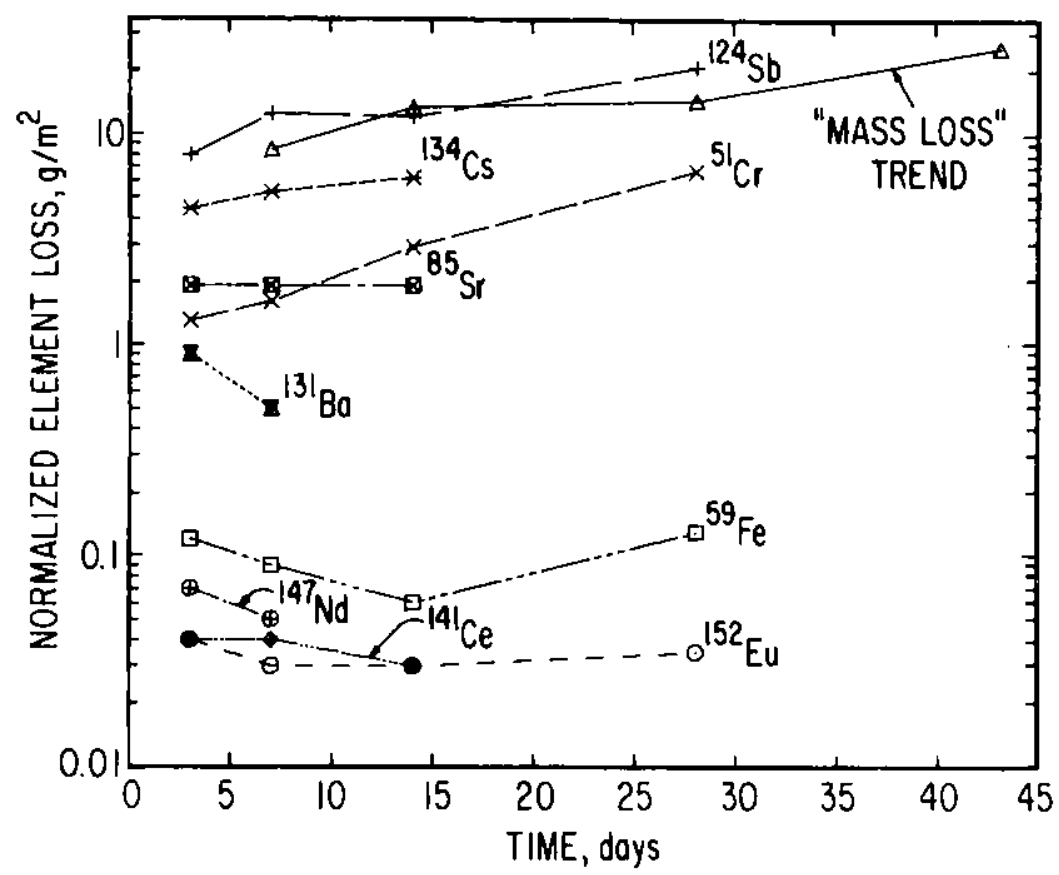

Fig. 9. Preliminary Leach Results for Activated SRL $211^{\star * *}$ Glass (Leach1ng at $90^{\circ} \mathrm{C}$ in deionized distilled water; MCC-1 tests)

Cold glass has been leached, and analyses have been completed through 91 days. Experiments that w11 extend through 364 days are in progress. The activated samples are being run simultaneously with the cold samples; thus, experiments with these two materials have been completed through 91 days. However, sample analyses have been delayed. Experiments on activated samples are planned to continue throigh 364 days. Spiked experiments, complete through 91 days, w111 continue for 364 days.

The neutron-activated-and-spiked leachates are being analyzed by the University of Missour1, using gamra-ray counting. Several reports have been received, but since there have been problems in normalizing the solids counting procedures, these results have been delayed. Leachate counting has proceeded without any such problems. Until analyses are complete, comparisons will be deferred.

\section{c. SRL 131 and SRL $131^{*}$ Glass}

The SRL 131 glass is the current SRL reference frit glass, and leach tests are in progress to determine the leaching behavior of both this frit glass and this glass containing about 3 wt \% waste additives of minor 
Table 6. Summary of Test Conditions, Leachate Analytical Results, and Weight Loss Rates for SRL $211^{\star \star *}$ Glass

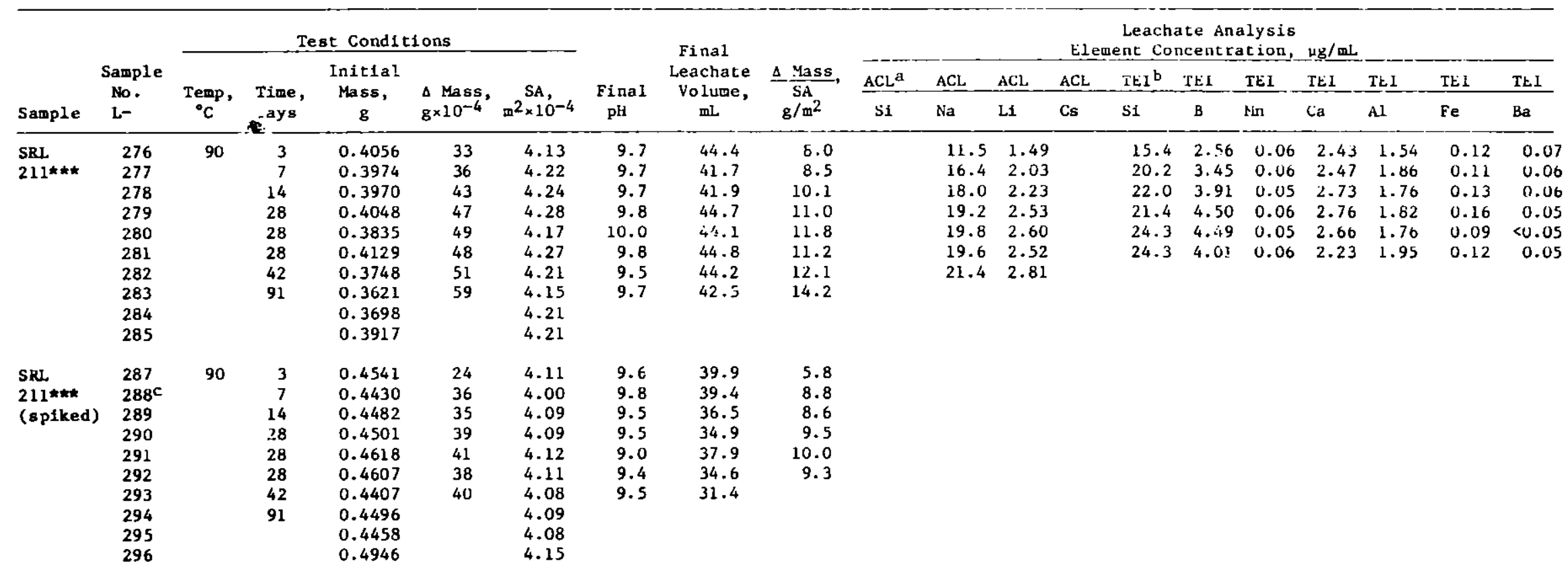


Table 6 (contd)

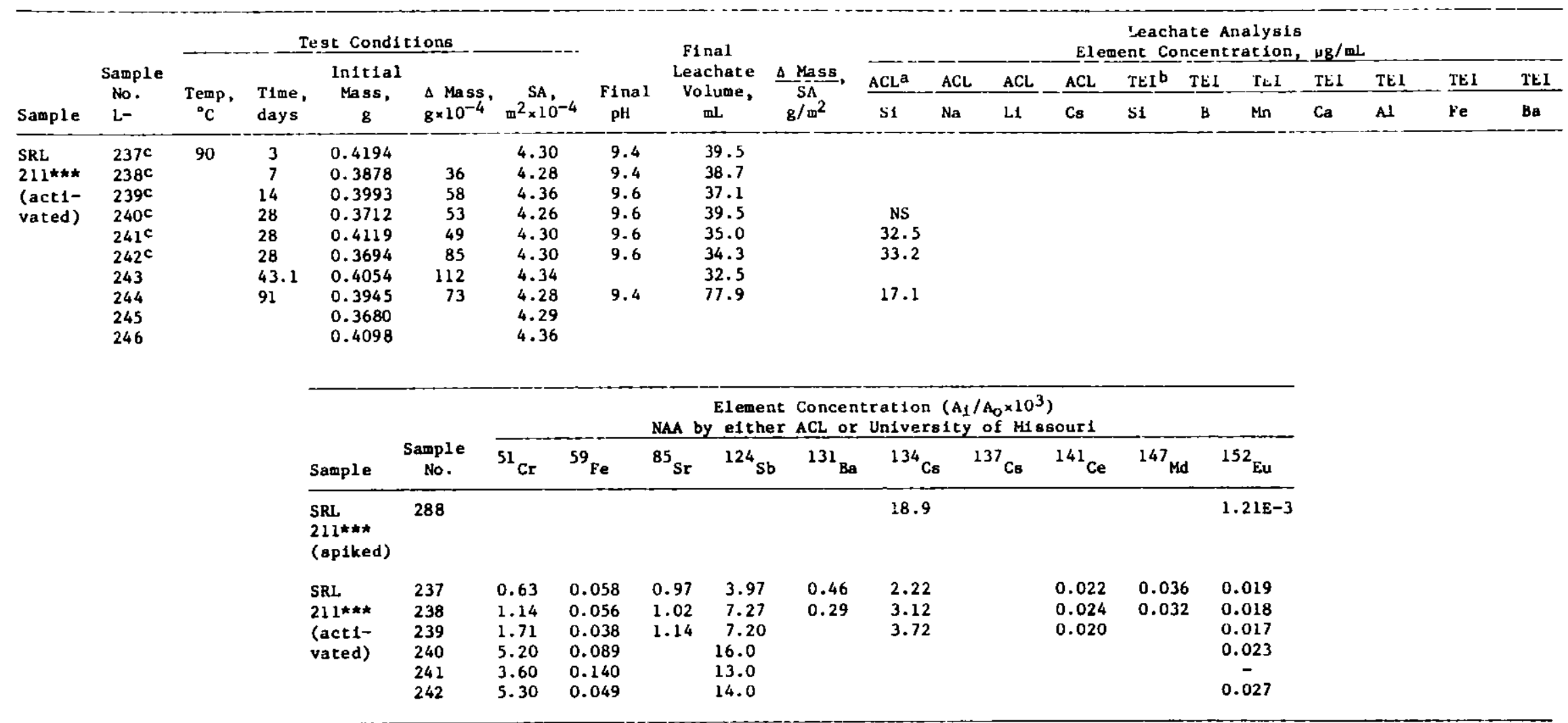

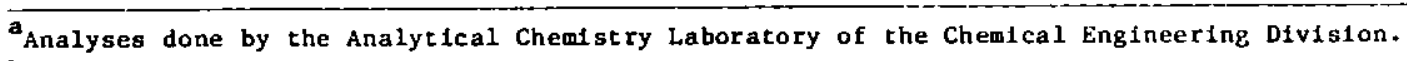

Analyses done by Trace Elements, Inc.

$c_{\text {Element concentrations are given at the end of this table. }}$ 
Table 7. Summary of Normalized Leach Rates Calculated from Vata in Table 6 for SRL $211 * * *$ Glass. Temperature $=90^{\circ} \mathrm{C}$.

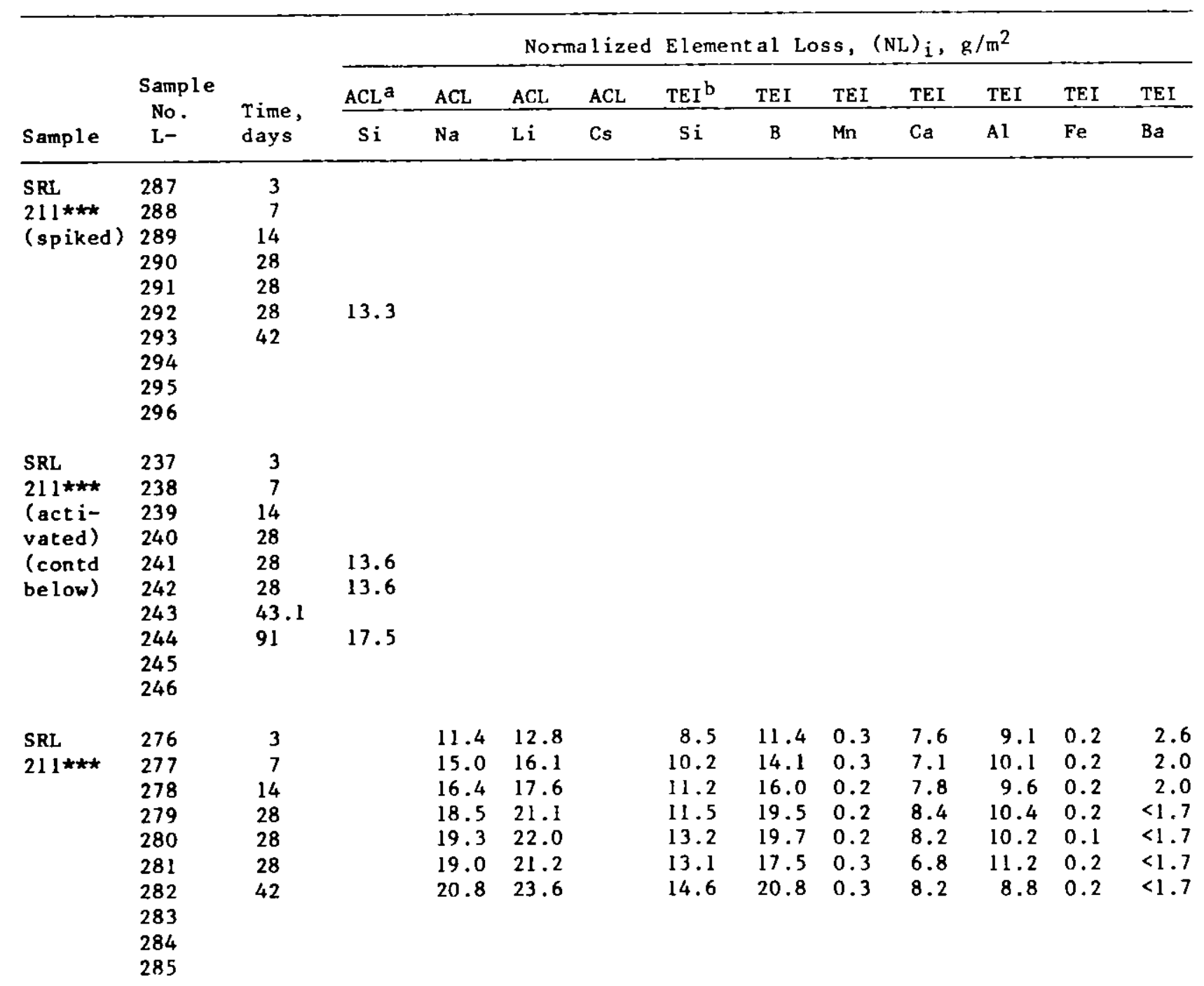


Table 7 (contd)

\begin{tabular}{|c|c|c|c|c|c|c|c|c|c|c|c|c|c|c|c|c|c|}
\hline \multirow[b]{2}{*}{ Sample } & \multirow{2}{*}{$\begin{array}{c}\text { Sample } \\
\text { No. } \\
\text { L- }\end{array}$} & \multirow[b]{2}{*}{$\begin{array}{l}\text { Time, } \\
\text { days }\end{array}$} & \multicolumn{15}{|c|}{ Normalized Elemental Loss, $(\mathrm{NL})_{\mathrm{i}}, \mathrm{g} / \mathrm{m}^{2}$} \\
\hline & & & ${ }^{51} \mathrm{Cr}$ & ${ }^{59} \mathrm{Fe}$ & ${ }^{85} \mathrm{Sr}$ & ${ }^{103} \mathrm{Ru}$ & ${ }^{106} \mathrm{Ru}$ & $131 \mathrm{Ba}$ & ${ }^{133} \mathrm{Ba}$ & ${ }^{134} \mathrm{Cs}$ & ${ }^{137} \mathrm{Cs}$ & ${ }^{39} \mathrm{Ce}$ & ${ }^{141} \mathrm{Ce}$ & 147 Nd & ${ }^{152} \mathrm{Eu}$ & ${ }^{154} \mathrm{Eu}$ & ${ }^{124} \mathrm{Sb}$ \\
\hline $\begin{array}{l}\text { SRL } \\
211 \star \star \star \\
\text { (spiked) }\end{array}$ & $\begin{array}{l}287 \\
288 \\
289 \\
290 \\
291 \\
292 \\
293 \\
294 \\
295 \\
296\end{array}$ & $\begin{array}{r}3 \\
7 \\
14 \\
28 \\
28 \\
28 \\
42\end{array}$ & & & & & 0.09 & & & & $\begin{array}{r}9.3 \\
20.5\end{array}$ & & $1.7 \mathrm{E}-3$ & & $\begin{array}{l}1.8 E-3 \\
4.8 E-3\end{array}$ & & \\
\hline $\begin{array}{l}\text { SRL } \\
211 \star \star \star \\
\text { (acti- } \\
\text { vated) }\end{array}$ & $\begin{array}{l}237 \\
238 \\
239 \\
240 \\
241 \\
242 \\
243 \\
244 \\
245 \\
246\end{array}$ & $\begin{array}{l}3 \\
7 \\
14 \\
28 \\
28 \\
28 \\
43.1 \\
91\end{array}$ & $\begin{array}{l}1.3 \\
1.6 \\
2.9 \\
6.9 \\
5.6 \\
7.4\end{array}$ & $\begin{array}{l}0.12 \\
0.09 \\
0.06 \\
0.12 \\
0.21 \\
0.07\end{array}$ & $\begin{array}{l}1.9 \\
1.9 \\
1.9\end{array}$ & & & $\begin{array}{l}0.9 \\
0.5\end{array}$ & & $\begin{array}{l}4.4 \\
5.3 \\
6.2\end{array}$ & & & $\begin{array}{l}0.04 \\
0.04 \\
0.03\end{array}$ & $\begin{array}{l}0.07 \\
0.05\end{array}$ & $\begin{array}{c}0.04 \\
0.03 \\
0.03 \\
0.03 \\
-- \\
0.04\end{array}$ & & $\begin{array}{r}7.9 \\
12.4 \\
12.0 \\
21.4 \\
20.0 \\
20.8\end{array}$ \\
\hline
\end{tabular}

Analyses done by the Analytical Chemistry Laboratory of the Chemical Engineering Division.

banalyses done by Trace Elements, Inc. 
elements (SRL 131*), as shown in Table 3. Tests are being done on cold glass, activated glass, and spiked $\left({ }^{85} \mathrm{Sr}, 137_{\mathrm{Cs}}, 133_{\mathrm{Ba}}, 141_{\mathrm{Ce}}, 152 \mathrm{Eu}\right)$ glass. The tests have been completed through 150 days (data available only through 91 days), and preliminary results (Tables 8 and 9) Indicate that a complete description of the leaching behavior of this glass will be possible.

\section{d. PNL 76-68 Glass}

The 76-68 glass supplied by the Materials Characterization Center (MCC) contains no europium and too much cesium to be useful for analysis by the NAA technique. Therefore, a new batch of 76-68 glass has been made and will be submitted for activation. This should allow the determination of noble metal and rare earth elements (which cannot be measured with spectroscopic techniques).

From the previously described leach tests, the usefulness of radioisotopic tracers for measuring leach rates will be qualified. Additionally, the behavior of several types of glasses upon contact with water will be observed. This behaviorial information can be used to demonstrate elemental release from the glass and to study the mechan 1 sm by which the glass is at tacked. However conclustons and qualifications must await completion of all current leach tests. Data presented in Tables 4 to 9 are preliminary and should be treated as such.

\section{Auxiliary Leach Studies}

\section{a. SYNROC}

Analyses have been completed on 97 solid samples submitted for leach testing. The samples, leaching conditions, and results are shown in Table 10. The SYNROC composition is shown in Table 11.

The solid samples were leached according to MCC-1 static leach test procedures, and the normalized leach rates, $(N L)_{i}$, were obtained in the following fashion:

Nonactivated Samples

$$
(N L)_{1}=\frac{M_{1}}{f_{1}-S A}
$$

where $M_{1}$ is the mass of element " 1 " In the leachate

$f_{1}$ is the mass fraction of element " $i$ " in the or Iginal solid

SA is the surface area $\left(m^{2}\right)$ of the sample 
Table 8. Summary of Test Conditions, Leachate Analytical Results, and Weight Loss Rates for SRL 131 Glass under Study

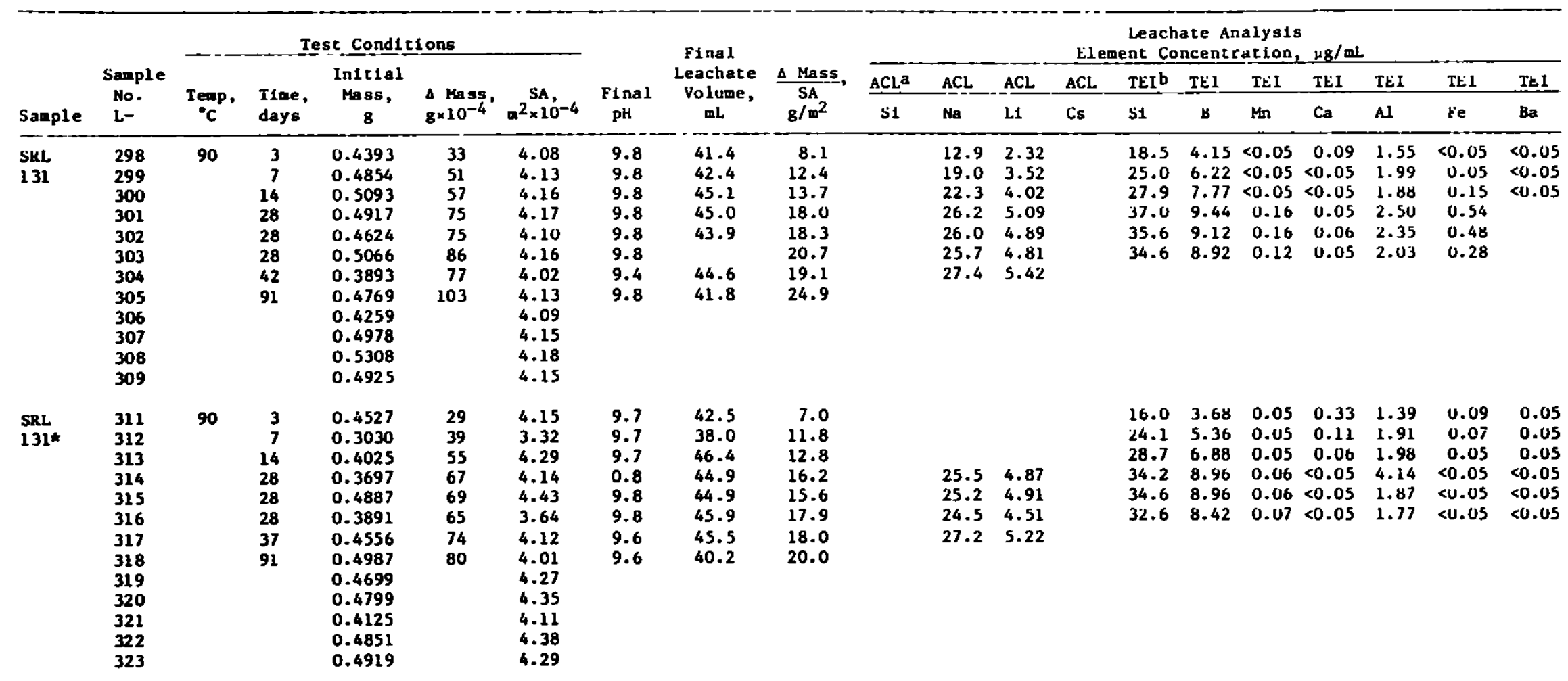


Table 8 (contd)

\begin{tabular}{|c|c|c|c|c|c|c|c|c|c|c|c|c|c|c|c|c|c|c|c|c|}
\hline \multirow[b]{3}{*}{ Saaple } & \multirow{3}{*}{$\begin{array}{l}\text { Smple } \\
\text { L- }\end{array}$} & \multicolumn{5}{|c|}{ Tesc Cond1 tions } & \multirow{3}{*}{\multicolumn{2}{|c|}{$\begin{array}{l}\text { Final } \\
\text { Leachate } \\
\text { Volume, } \\
\text { ml. }\end{array}$}} & \multirow{3}{*}{$\begin{array}{c}\Delta \frac{M a s s}{S A} . \\
g / m^{2}\end{array}$} & \multicolumn{11}{|c|}{$\begin{array}{l}\text { Leachate Analysis } \\
\text { Element Concentration, ug/mL }\end{array}$} \\
\hline & & Teap, & Tine, & $\begin{array}{c}\text { In1tial } \\
\text { Mass, }\end{array}$ & A Hass, & sa, & & & & $A C L$ & $\mathrm{ACL}$ & ACL & $\mathrm{ACL}$ & {$\left[E_{1}{ }^{b}\right.$} & TEI & TEl & TEE I & TË I & IEI & IEL \\
\hline & & c & days & 8 & $8 \times 10^{-4}$ & $n^{2} \times 10^{-4}$ & & & & si & $\mathrm{Na}$ & LI & cs & si & B & Mn & $\mathrm{Ca}$ & Al & $\mathrm{re}$ & Ba \\
\hline $\begin{array}{l}\text { Sol. } \\
131 . \\
\text { (acti- } \\
\text { vated) }\end{array}$ & $\begin{array}{l}327 \\
328 \\
329 \\
330 \\
331 \\
333\end{array}$ & 90 & $\begin{array}{l}2.25 \\
7 \\
14 \\
28 \\
28\end{array}$ & $\begin{array}{l}0.3112 \\
0.3986 \\
0.3989 \\
0.4595 \\
0.4067\end{array}$ & $\begin{array}{l}21 \\
15 \\
24 \\
39 \\
49\end{array}$ & $\begin{array}{l}3.77 \\
3.86 \\
3.90 \\
4.33 \\
4.21\end{array}$ & $\begin{array}{l}9.2 \\
5.2\end{array}$ & $\begin{array}{l}35.4 \\
34.0\end{array}$ & $\begin{array}{r}5.6 \\
3.9 \\
6.2 \\
9.0 \\
11.6\end{array}$ & $\begin{array}{l}41.2 \\
43.2\end{array}$ & & & & & & & & & & \\
\hline $\begin{array}{l}\text { SRL } \\
131 \\
\text { (splked) }\end{array}$ & $\begin{array}{l}339 \\
340 \\
341 \\
342 \\
343 \\
344 \\
345 \\
346\end{array}$ & 90 & $\begin{array}{r}3 \\
7 \\
14 \\
28 \\
28 \\
28 \\
42\end{array}$ & $\begin{array}{l}0.5174 \\
0.4869 \\
0.4842 \\
0.4772 \\
0.4866 \\
0.4965 \\
0.4733 \\
0.4651\end{array}$ & $\begin{array}{l}17 \\
32 \\
54 \\
56 \\
50 \\
58 \\
51\end{array}$ & $\begin{array}{l}4.35 \\
4.26 \\
4.27 \\
4.28 \\
4.29 \\
4.29 \\
4.27 \\
4.26\end{array}$ & $\begin{array}{l}9.5 \\
9.3 \\
9.4 \\
9.2 \\
9.0 \\
9.3 \\
9.2\end{array}$ & $\begin{array}{l}39.5 \\
39.0 \\
37.7 \\
34.0 \\
34.2 \\
34.5 \\
31.9\end{array}$ & $\begin{array}{r}3.9 \\
7.5 \\
12.7 \\
13.1 \\
11.7 \\
13.5 \\
11.9\end{array}$ & $\begin{array}{l}43.3 \\
36.2 \\
46.5\end{array}$ & & & & & & & & & & \\
\hline
\end{tabular}

Analyaea done by the Analytical Chematry Laboratory of the Chemical Engineering Division.

Analyees done by Trace Elements, Inc. 
Table 9. Summary of Normalized Leach Rates Calculated from Data in Table 8 for SRL 131 Glass. Temperature $=90^{\circ} \mathrm{C}$.

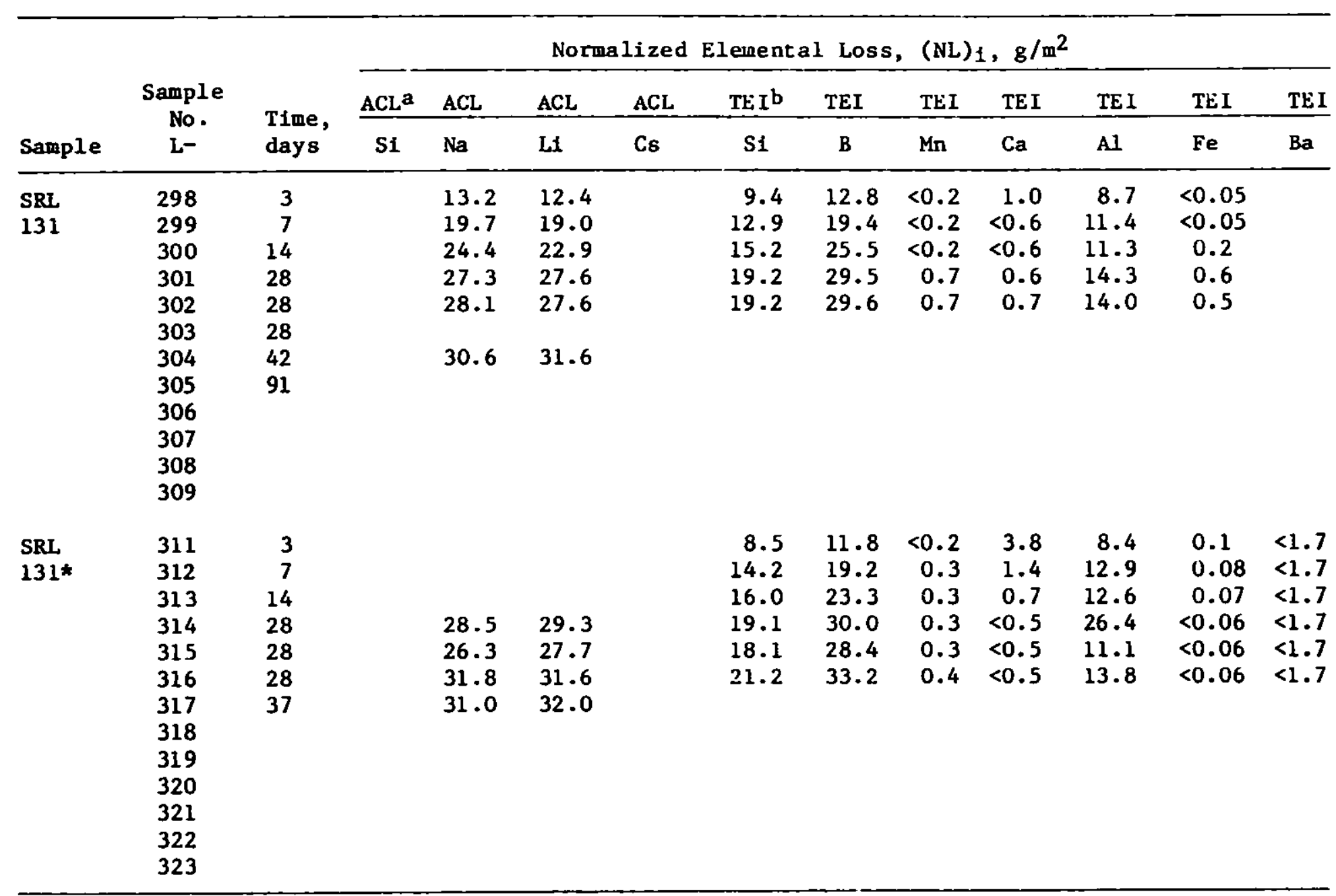

\footnotetext{
analyses done by the Analytical Chemistry Laboratory of the Chemical Engineering Division.

banalyses done by Trace Elements, Inc.
} 
Table 10. Test Conditions and Leach Resultsa

for SYNROC Samples

\begin{tabular}{|c|c|c|c|c|c|c|c|c|c|c|c|}
\hline smple & $\begin{array}{c}\text { Sample } \\
\text { Ho. }\end{array}$ & $\begin{array}{l}\text { Time, } \\
\text { days }\end{array}$ & ${ }^{\text {Temp. }} \mathrm{C}$ & $\begin{array}{c}\text { Initial } \\
\text { Mass, } \\
\text { g }\end{array}$ & $\begin{array}{l}\Delta \text { Mss, } \\
\mathrm{g} \times 10^{-4}\end{array}$ & $\mathrm{~m}^{2} \times 10^{-4}$ & $\begin{array}{c}\text { Final } \\
\text { pH }\end{array}$ & $\begin{array}{c}\text { Final } \\
\text { Leachate } \\
\text { Volume, } \\
\text { mL }\end{array}$ & $\frac{\Delta \text { Mass }}{\frac{\mathrm{SA}}{\mathrm{g} / \mathrm{m}^{2}}}$ & Leachate ${ }^{b}$ & $\begin{array}{l}\text { Method } \\
\text { of } \\
\text { Analysis }\end{array}$ \\
\hline$\left.\right|_{-10} ^{B 9 A-9}$ & $\begin{array}{l}L-347 \\
L-348 \\
L-349 \\
L-350 \\
L-351 \\
L-352 \\
L-353 \\
L-354 \\
L-355 \\
L-356 \\
L-357 \\
L-358 \\
L-359 \\
L-360 \\
L-361 \\
L-362 \\
L-363 \\
L-364 \\
L-365 \\
L-366 \\
L-367\end{array}$ & $\begin{array}{l}1 \\
4.75 \\
7 \\
14 \\
28 \\
28 \\
1 \\
28 \\
28 \\
28 \\
28 \\
4.75 \\
7 \\
14 \\
28 \\
1\end{array}$ & $\int_{40}^{90}$ & $\begin{array}{l}0.2942 \\
0.2586 \\
0.2689 \\
0.3014 \\
0.3044 \\
0.3052 \\
0.2914 \\
0.2856 \\
0.3131 \\
0.3263 \\
0.2778 \\
0.2746 \\
0.2927 \\
0.3145 \\
0.2904 \\
0.2618 \\
0.3158 \\
0.3003 \\
0.2934 \\
0.3037 \\
0.2967\end{array}$ & $\begin{array}{l}4 \\
4 \\
3 \\
4 \\
2 \\
2 \\
6 \\
9 \\
2 \\
2 \\
1 \\
3 \\
3 \\
4 \\
1 \\
2 \\
2 \\
1 \\
3 \\
1 \\
3\end{array}$ & $\begin{array}{l}2.01 \\
2.01 \\
2.01 \\
2.01 \\
2.01 \\
2.01 \\
2.01 \\
1.97 \\
1.99 \\
2.00 \\
1.98 \\
1.94 \\
1.94 \\
1.95 \\
1.93 \\
1.95 \\
1.98 \\
1.94 \\
1.92 \\
1.95 \\
1.94\end{array}$ & $\begin{array}{l}5.1 \\
5.1 \\
4.6 \\
4.8 \\
4.8 \\
4.4 \\
4.9 \\
5.7 \\
4.9 \\
9.4 \\
3.6 \\
4.7 \\
4.7 \\
4.1 \\
5.3 \\
4.8 \\
4.8 \\
9.3 \\
3.7 \\
4.7 \\
4.8\end{array}$ & $\begin{array}{r}19.8 \\
19.7 \\
20.0 \\
21.1 \\
22.8 \\
22.2 \\
10.0 \\
10.2 \\
22.2 \\
21.2 \\
19.9 \\
20.1 \\
19.6 \\
24.2 \\
19.9 \\
20.0 \\
22.2 \\
20.1 \\
21.4 \\
20.2 \\
9.9\end{array}$ & & $\left.\right|_{\text {SW }} ^{\text {B }}$ & $D C P / A A$ \\
\hline
\end{tabular}


Table 10 (contd)

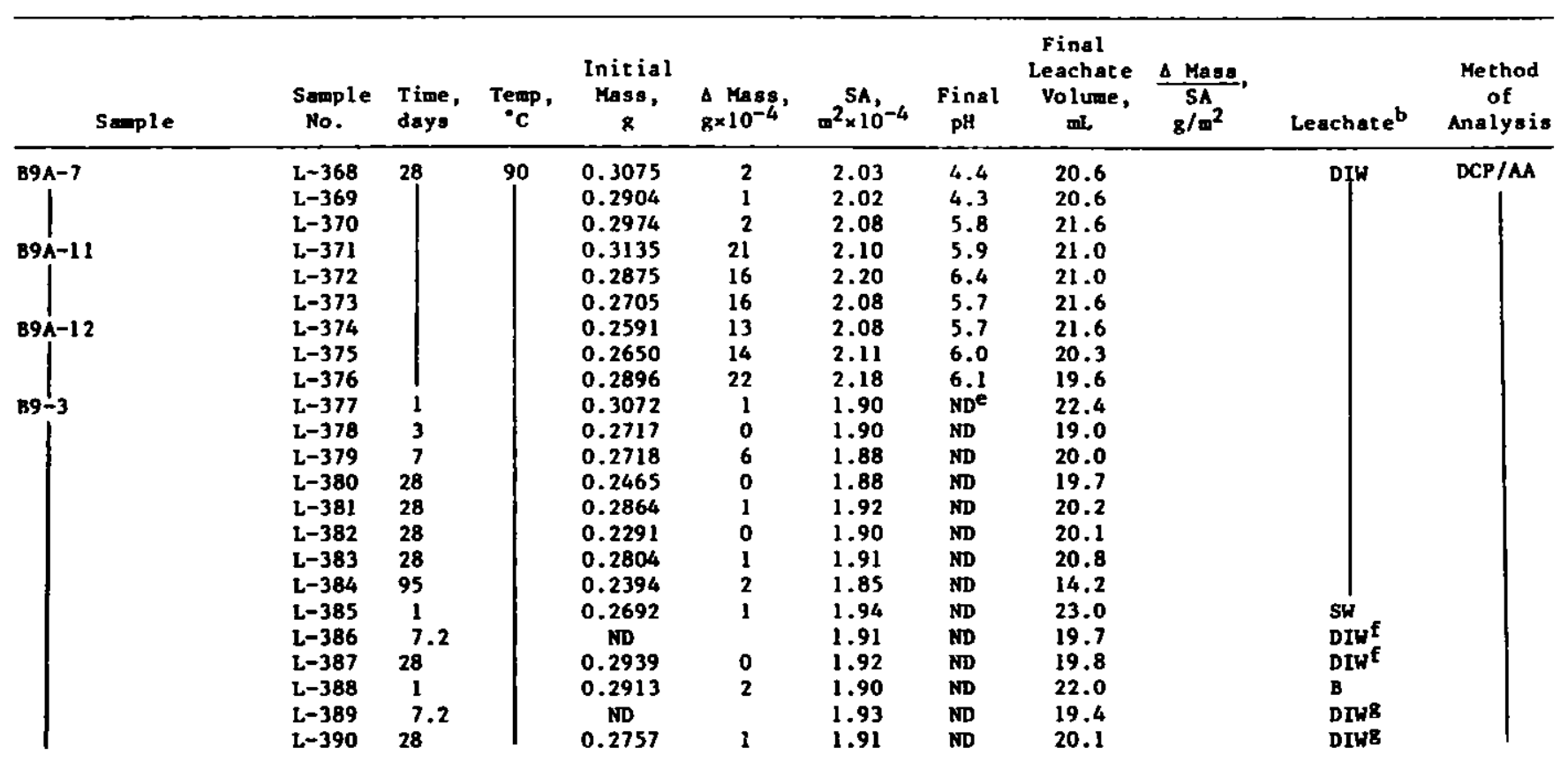


Table 10 (contd)

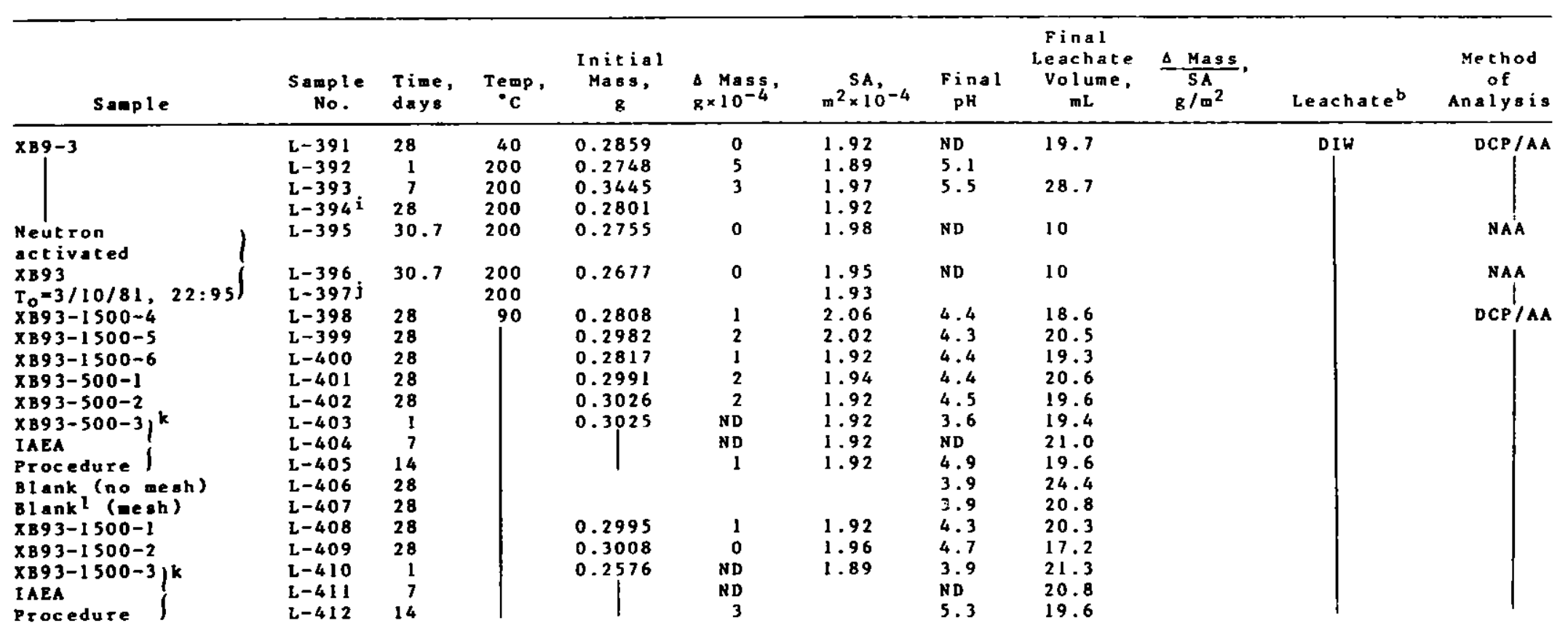


Table 10 (contd)

\begin{tabular}{|c|c|c|c|c|c|c|c|c|c|c|c|}
\hline Sample & $\begin{array}{c}\text { Sample } \\
\text { No. }\end{array}$ & $\begin{array}{l}\text { Time, } \\
\text { days }\end{array}$ & $\underset{\mathrm{C}}{\text { Temp, }}$ & $\begin{array}{c}\text { Initial } \\
\text { Mass. } \\
\text { g }\end{array}$ & $\begin{array}{l}\Delta \text { Mass, } \\
g \times 10^{-4}\end{array}$ & $\mathrm{~m}^{2} \times 10^{-4}$ & $\begin{array}{c}\text { Final } \\
\text { pH }\end{array}$ & $\begin{array}{l}\text { Final } \\
\text { Leachate } \\
\text { volume, } \\
\text { mL }\end{array}$ & $\frac{\Delta \text { Mass }}{\mathrm{sA} / \mathrm{m}^{2}}$ & Leachateb & $\begin{array}{l}\text { Method } \\
\text { of } \\
\text { Ansiysis }\end{array}$ \\
\hline $\begin{array}{l}\text { XB93-500-He-1 } \\
\text { XB93-500-He-2, I }\end{array}$ & $\begin{array}{l}L-413 \\
L-414\end{array}$ & $\begin{array}{r}3 \\
28\end{array}$ & $\begin{array}{l}90 \\
90\end{array}$ & $\begin{array}{l}0.2631 \\
0.2928\end{array}$ & 0 & $\begin{array}{l}1.90 \\
1.90\end{array}$ & $\begin{array}{l}4.6 \\
4.5\end{array}$ & $\begin{array}{l}24.3 \\
20.6\end{array}$ & & $\begin{array}{l}\text { DIW } \\
\text { DIW }\end{array}$ & DCP / AA \\
\hline XB93-500-He-3 & $L-415$ & ext & & & & & & & & DIW & \\
\hline XB93-500-He-4 $\left\{\begin{array}{l}\mathrm{I} \\
\mathrm{A} \\
\mathrm{A}\end{array}\right.$ & $\begin{array}{l}\mathrm{L}-416 \\
\mathrm{~L}-417^{\mathrm{m}}\end{array}$ & $\begin{array}{c}3 \\
\operatorname{rot}=1 \\
28\end{array}$ & 200 & 0.3109 & & 1.90 & 4.9 & 18.5 & & $\begin{array}{l}\text { DIW } \\
\text { DIW }\end{array}$ & \\
\hline $\begin{array}{l}\text { XB93-1 500-He-4 } \\
\text { XB93-1500-He-5 } \\
\text { XB93-1500-He-6 } \\
\text { XB93-1500-He-7a } \\
\text { XB93-1500-He-7b }\end{array}$ & $\begin{array}{l}\mathrm{L}-418 \\
\mathrm{~L}-419 \\
\mathrm{~L}-420 \\
\mathrm{~L}-421^{\mathrm{m}} \\
\mathrm{L}-422\end{array}$ & $\begin{array}{r}3 \\
28 \\
3 \\
28 \\
3\end{array}$ & $\begin{array}{r}90 \\
90 \\
200 \\
200 \\
90\end{array}$ & $\begin{array}{l}0.2680 \\
0.2956 \\
0.3042 \\
0.1736 \\
0.1355\end{array}$ & $\begin{array}{r}+1 \\
1 \\
3 \\
1\end{array}$ & $\begin{array}{l}1.90 \\
1.90 \\
1.91 \\
1.17 \\
0.93\end{array}$ & $\begin{array}{l}4.3 \\
4.5 \\
3.2 \\
4.4\end{array}$ & $\begin{array}{l}21.4 \\
20.2 \\
19.4 \\
23.3\end{array}$ & & $\begin{array}{l}\text { DIW } \\
\text { DIW } \\
\text { DIW } \\
\text { DIW } \\
\text { DIW }\end{array}$ & \\
\hline $\begin{array}{l}\text { XB93-1000 pCi-He-8 } \\
\text { XB93-1000-uC }{ }^{i}-\text { He-9 } \\
\text { IAEA procedure } \\
\text { XB93 (control) } \\
\text { Blank }\end{array}$ & $\begin{array}{l}L-427 \\
L-428 \\
L-429 \\
L-430 \\
L-431\end{array}$ & $\begin{array}{l}14.5 \\
3 \\
25 \\
28\end{array}$ & $\begin{array}{r}145 \\
90 \\
90 \\
90\end{array}$ & $\begin{array}{l}0.3158 \\
0.2799 \\
0.2814\end{array}$ & $\begin{array}{l}2 \\
1\end{array}$ & $\begin{array}{l}1.92 \\
1.92 \\
1.89\end{array}$ & $\begin{array}{l}9.5 \\
9.1 \\
4.5\end{array}$ & $\begin{array}{l}19.9 \\
20.0 \\
19.6\end{array}$ & & $\begin{array}{l}\text { DIW } \\
\text { SW } \\
\text { SW } \\
\text { DIW }\end{array}$ & \\
\hline
\end{tabular}


Table 10 (contd)

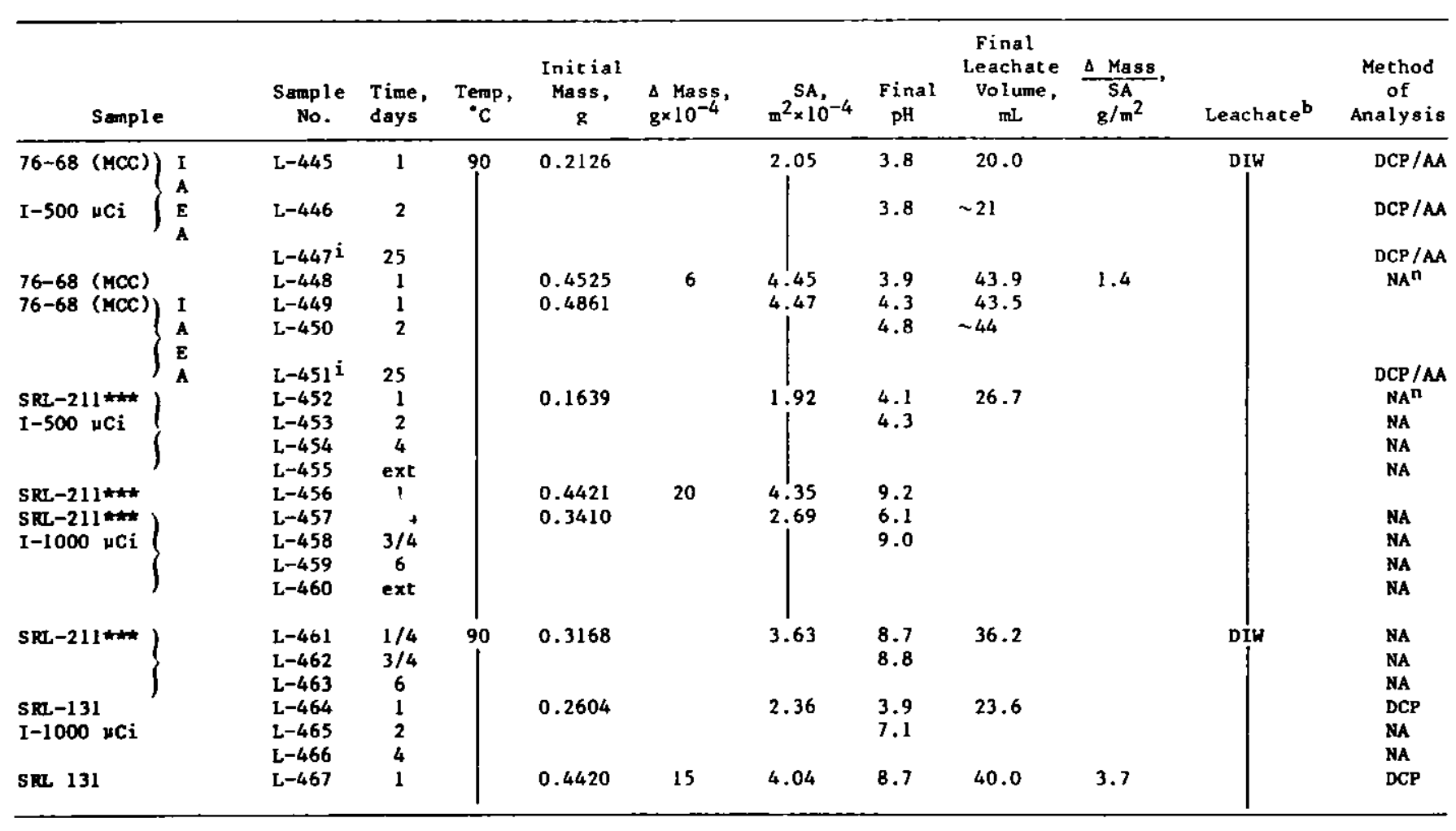


Table 10 (contd)

\begin{tabular}{|c|c|c|c|c|c|c|c|c|c|c|c|c|c|c|c|c|c|c|c|c|c|}
\hline \multirow[b]{2}{*}{ Sample } & \multirow{2}{*}{$\begin{array}{c}\text { Sample } \\
\text { No. }\end{array}$} & \multicolumn{2}{|c|}{ Cs } & \multicolumn{2}{|c|}{$\mathrm{Ca}$} & \multicolumn{2}{|c|}{$\mathrm{Ba}$} & \multicolumn{2}{|c|}{ Al } & \multicolumn{2}{|c|}{ Mo } & \multicolumn{2}{|c|}{$\mathrm{Sr}^{c}$} & \multicolumn{2}{|c|}{$T i$} & \multicolumn{2}{|c|}{ Si } & \multicolumn{2}{|r|}{$\mathrm{Ni}$} & \multicolumn{2}{|c|}{$\mathrm{Fe}$} \\
\hline & & $\mathrm{mg} / \mathrm{L}$ & (NL) & $\mathrm{mg} / \mathrm{L}$ & (NL) & $\mathrm{mg} / \mathrm{L}$ & (NL) & $\mathrm{mg} / \mathrm{L}$ & (NL) & $\mathrm{mg} / \mathrm{L}$ & (NL) & $\mathrm{mg} / \mathrm{L}$ & (NL) & $\mathrm{mg} / \mathrm{L}$ & (NL) & $\mathrm{mg} / \mathrm{L}$ & (NL) & $\mathrm{mg} / \mathrm{L}$ & (NL) & $\mathrm{mg} / \mathrm{L}$ & (N) \\
\hline \multirow[t]{7}{*}{$\mathrm{B} 9 \mathrm{~A}-8$} & $\begin{array}{l}L-347 \\
L-348\end{array}$ & 0.02 & 0.5 & $\begin{array}{l}0.26 \\
0.40\end{array}$ & $\begin{array}{l}0.3 \\
0.5\end{array}$ & $\begin{array}{l}0.28 \\
0.85\end{array}$ & $\begin{array}{l}0.34 \\
1.02\end{array}$ & $\begin{array}{l}0.09 \\
0.54\end{array}$ & $\begin{array}{l}0.13 \\
0.80\end{array}$ & $\begin{array}{l}0.58 \\
1.54\end{array}$ & $\begin{array}{r}8.7 \\
13.6\end{array}$ & & & & $<0.02$ & $\begin{array}{r}0.27 \\
<0.05\end{array}$ & 133 & 0.20 & 9.0 & 0.09 & \\
\hline & $\mathrm{L}-349$ & 0.10 & 2.3 & 0.56 & 0.7 & 1.19 & 1.45 & 0.73 & 1.10 & 1.56 & 14.0 & & & & & $\begin{array}{l}0.07 \\
1.12\end{array}$ & 557 & 0.26 & 11.8 & 0.13 & \\
\hline & $L-350$ & 0.16 & 3.9 & 0.47 & 0.6 & 1.46 & 1.88 & 0.95 & 1.50 & 0.99 & 9.4 & & & & & 1.32 & 693 & 0.20 & 9.5 & 0.09 & \\
\hline & L-351 & 0.20 & 5.3 & 0.55 & 0.8 & 1.56 & 2.17 & 1.07 & 1.83 & 1.24 & 12.7 & 0.075 & 2.2 & & & 1.49 & 845 & 0.24 & 12.4 & 0.16 & \\
\hline & $L-352$ & $<0.02$ & $<0.5$ & 0.22 & 0.3 & 0.37 & 0.50 & 0.24 & 0.40 & 0.84 & 8.4 & & & & & 0.32 & 177 & 0.16 & 8.0 & 0.08 & 1 \\
\hline & $L-353$ & $\mathrm{NS}^{\mathrm{d}}$ & & 2.51 & 1.6 & 1.00 & 0.61 & 2.28 & 1.71 & 4.16 & 18.7 & & & & & 6.88 & 1711 & 0.05 & 1.1 & 0.08 & 0 \\
\hline & $\mathrm{L}-354$ & 2.44 & 28.8 & 3.00 & 2.0 & 8.58 & 5.40 & 0.74 & 0.58 & 3.03 & 14.1 & & & & & 6.69 & & 0.07 & 2.6 & 0.12 & \\
\hline \multirow[t]{3}{*}{$B 9 A-9$} & $L-355$ & 0.21 & 5.5 & 0.37 & 0.5 & 1.65 & 2.26 & 1.26 & 2.12 & 0.74 & 7.4 & 0.075 & 2.2 & & & 1.48 & 826 & 0.17 & 8.6 & 0.06 & \\
\hline & $L-356$ & 0.19 & 4.7 & $<0.05$ & $<0.07$ & 0.20 & 0.26 & 0.14 & 0.22 & 0.53 & 5.1 & 0.007 & 0.2 & & & 32.9 & & $<0.05$ & $<2.4$ & $<0.05$ & $<0$ \\
\hline & {$[-357$} & $\mathrm{ND}^{\mathrm{e}}$ & & $<0.05$ & $<0.07$ & 0.43 & 0.53 & 1.60 & 2.44 & 0.75 & 6.8 & 0.07 & 2.2 & & & 0.23 & 456 & 0.24 & 11.3 & 1.10 & \\
\hline \multirow[t]{10}{*}{ B9A-10 } & $L-358$ & 0.13 & 3.1 & 0.10 & 0.1 & 1.44 & 1.83 & 0.67 & 1.05 & 0.78 & 7.3 & & & & & 0.88 & 445 & 0.15 & 6.9 & 0.32 & \\
\hline & $L-359$ & 0.18 & 4.2 & 0.16 & 0.2 & 1.67 & 2.07 & 0.80 & 1.22 & 0.80 & 7.3 & & & & & 0.81 & 503 & 0.16 & 7.4 & 0.42 & \\
\hline & $L-360$ & 0.21 & 6.1 & 0.16 & 0.25 & 1.01 & 1.54 & 0.94 & 1.76 & 0.71 & 7.9 & & & & & 0.98 & 608 & 0.15 & 8.5 & 0.31 & \\
\hline & L-361 & 0.34 & 8.2 & 0.17 & 0.22 & 2.51 & 3.17 & 1.21 & 1.88 & 0.92 & 8.6 & & & & & 1.24 & 639 & 0.18 & 8.4 & 0.39 & \\
\hline & L-362 & 0.33 & 7.9 & 0.18 & 0.23 & 2.50 & 3.14 & 1.22 & 1.89 & 0.90 & 8.3 & & & & & 1.30 & 667 & 0.17 & 7.9 & 0.37 & \\
\hline & L-363 & 0.37 & 9.7 & 0.15 & 0.21 & 2.91 & 4.00 & 1.14 & 1.93 & 0.90 & 9.1 & & & & & 1.43 & 802 & 0.16 & 8.2 & 0.33 & \\
\hline & $L-364$ & 0.18 & 4.3 & $<0.05$ & $<0.07$ & 0.10 & 0.13 & 0.09 & 0.14 & 0.47 & 4.4 & & & & & 31.8 & & $<0.05$ & $<2.4$ & $<0.05$ & \\
\hline & $\mathrm{L}-365$ & & & 0.08 & 0.10 & 0.73 & 0.93 & 1.81 & 2.84 & 1.07 & 10.0 & & & & & 0.46 & & 0.32 & & 2.05 & \\
\hline & $L-366$ & 0.03 & 0.7 & $0.1 \mathrm{i}$ & 0.14 & 0.72 & 0.91 & 0.29 & 0.45 & 0.73 & 6.8 & & & & & 0.31 & 160 & 0.19 & 9.0 & 0.36 & \\
\hline & $L-367$ & 1.0 & 11.8 & 0.53 & 0.67 & 0.17 & 0.11 & 0.07 & 0.05 & 2.81 & 12.9 & & & & & 7.78 & & $<0.05$ & $<2.4$ & 0.05 & \\
\hline
\end{tabular}


Table 10 (contd)

\begin{tabular}{|c|c|c|c|c|c|c|c|c|c|c|c|c|c|c|c|c|c|c|c|c|c|}
\hline \multirow[b]{2}{*}{ Sample } & \multirow{2}{*}{$\begin{array}{l}\text { Esmple } \\
\text { No. }\end{array}$} & \multicolumn{2}{|c|}{ Cs } & \multicolumn{2}{|c|}{$\mathrm{Ca}$} & \multicolumn{2}{|c|}{$\mathrm{Ba}$} & \multicolumn{2}{|c|}{ A1 } & \multicolumn{2}{|c|}{ Mo } & \multicolumn{2}{|c|}{$\mathrm{Sr}^{\mathrm{c}}$} & \multicolumn{2}{|c|}{$\mathrm{Ti}$} & \multicolumn{2}{|c|}{ Si } & \multicolumn{2}{|c|}{$\mathrm{Ni}$} & \multicolumn{2}{|c|}{$\mathrm{Fe}$} \\
\hline & & $\mathrm{mg} / \mathrm{L}$ & (NL) & $\mathrm{mg} / \mathrm{L}$ & (NL) & $\mathrm{mg} / \mathrm{L}$ & (NL) & $\mathrm{mg} / \mathrm{L}$ & (NL) & $\mathrm{mg} / \mathrm{L}$ & (NL) & $\mathrm{mg} / \mathrm{L}$ & (NL) & $\mathrm{mg} / \mathrm{L}$ & (NL) & $\mathrm{mg} / \mathrm{L}$ & (NL) & $\mathrm{mg} / \mathrm{L}$ & (NL) & $\mathrm{mg} / \mathrm{L}$ & (NL) \\
\hline \multirow[t]{3}{*}{$B 9 A-7$} & L-368 & 0.86 & 20.3 & 0.36 & 0.46 & 3.40 & 4.23 & 1.42 & 2.17 & $<0.05$ & $<0.5$ & & & & $<0.02$ & 1.66 & 842 & 0.28 & 12.9 & $<0.05$ & $<0.7$ \\
\hline & $\mathrm{L}-369$ & 0.74 & 17.6 & 0.29 & 0.37 & 3.29 & 4.11 & 1.39 & 2.13 & $<0.05$ & $<0.5$ & & & & & 1.57 & 801 & 0.24 & 11.1 & $<0.05$ & $<0.7$ \\
\hline & $L-370$ & 0.14 & 27.5 & 0.33 & 0.43 & 3.76 & 4.15 & 0.26 & 0.41 & $<0.05$ & $<0.5$ & & & & & 1.94 & 1007 & 0.22 & 10.4 & $<0.05$ & $<0.7$ \\
\hline \multirow[t]{3}{*}{$B 9 A-11$} & $L-371$ & 33.3 & 737 & 2.57 & 3.24 & 6.62 & 8.11 & 0.19 & 0.29 & 22.5 & 202 & & & & & 3.15 & 1575 & $<0.05$ & $<2.4$ & $<0.05$ & $<0.7$ \\
\hline & $L-372$ & 21.3 & 606 & 2.64 & 3.17 & 7.10 & 8.31 & 0.15 & 0.22 & 19.5 & 168 & & & & & 3.31 & 1580 & $<0.05$ & $<2.4$ & $<0.05$ & $<0.7$ \\
\hline & $L-373$ & 24.3 & 507 & 2.61 & 3.41 & 8.04 & 10.23 & 0.15 & 0.23 & 15.6 & 146 & & & & & 2.85 & 1480 & $<0.05$ & $<2.4$ & $<0.05$ & $<0.7$ \\
\hline \multirow[t]{3}{*}{$B 9 A-12$} & L-374 & 16.7 & 409 & 2.89 & 3.83 & 6.95 & 8.97 & 0.15 & 0.24 & 12.1 & 115 & & & & & 2.98 & 1570 & $<0.05$ & $<2.4$ & 0.05 & $<0.7$ \\
\hline & L-375 & 19.9 & 445 & 3.16 & 3.83 & 6.58 & 7.76 & $<0 \quad 05$ & $<0.08$ & 12.1 & 105 & & & & & 3.67 & 1765 & $<0.05$ & $<2.4$ & $<0.05$ & $<0.7$ \\
\hline & L-376 & 20.8 & 435 & 7.96 & 9.01 & 9.74 & 10.73 & 0.17 & 0.23 & 13.1 & 106 & & & & & 5.69 & 2558 & $<0.05$ & $<2.4$ & $<0.05$ & $<0.7$ \\
\hline \multirow[t]{14}{*}{$B 9-3$} & L-377 & 0.09 & 2.5 & 0.13 & 0.19 & 0.15 & 0.22 & 0.08 & 0.14 & $<0.09$ & $<0.5$ & & & & & 0.08 & 47 & 0.10 & 5.4 & $<0.05$ & $<0.7$ \\
\hline & L-378 & 0.14 & 3.3 & 0.17 & 0.21 & 0.23 & 0.28 & 0.08 & 0.12 & $<0.05$ & $<0.5$ & & & & & 0.16 & 80 & 0.09 & 4.1 & $<0.05$ & $<0.7$ \\
\hline & L-379 & 0.19 & 4.7 & 0.21 & 0.28 & 0.28 & 0.37 & 0.09 & 0.14 & $<0.05$ & $<0.5$ & & & & & 0.21 & 112 & 0.09 & 4.4 & $<0.05$ & $<0.7$ \\
\hline & $L-380$ & 0.36 & 8.9 & 0.12 & 0.16 & 0.38 & 0.49 & 0.07 & 0.11 & $<0.05$ & $<0.5$ & & & & & 0.30 & 157 & 0.12 & 5.7 & 0.06 & 0.8 \\
\hline & $\mathrm{L}-381$ & 0.36 & 8.8 & 0.11 & 0.15 & 0.37 & 0.48 & $<0.05$ & $<0.08$ & $<0.05$ & $<0.5$ & & & & & 0.29 & 153 & 0.08 & 3.8 & $<0.05$ & $<0.7$ \\
\hline & $\mathrm{L}-382$ & 0.36 & 8.9 & 0.11 & 0.15 & 0.39 & 0.51 & $<0.05$ & $<0.08$ & $<0.05$ & $<0.5$ & 0.015 & 0.42 & & & 0.28 & 148 & 0.09 & 4.3 & $<0.05$ & $<0.7$ \\
\hline & $L-383$ & 1.13 & 28.6 & 0.35 & 0.48 & 0.98 & 1.31 & 0.70 & 1.15 & 0.09 & 0.88 & & & & & 0.86 & 226 & 0.55 & 27.2 & 0.06 & 0.8 \\
\hline & $L-384$ & 0.61 & 10.9 & & & & & & & & & & & & & & & & & & \\
\hline & $L-385$ & 0.09 & 2.5 & $<0.06$ & $<0.07$ & 0.08 & 0.12 & 0.07 & 0.13 & $<0.05$ & $<0.5$ & & & & & 32.6 & & $<0.05$ & & $<0.05$ & $<0.7$ \\
\hline & $\mathrm{L}-386$ & 0.21 & 5.0 & 0.29 & 0.38 & 0.33 & 0.42 & $<0.05$ & $<0.08$ & $<0.05$ & $<0.5$ & & & & & 0.19 & & 0.11 & & $<0.05$ & \\
\hline & L-387 & 0.53 & 12.7 & 0.36 & 0.74 & 0.57 & 0.72 & 0.11 & 0.17 & 0.05 & 0.5 & & & & & 0.49 & & 0.15 & & 0.13 & \\
\hline & $\mathrm{L}-388$ & ND & ND & $<0.05$ & $<0.07$ & 0.16 & 0.21 & 1.38 & & 0.26 & & & & & & 0.10 & & 0.33 & & 1.10 & \\
\hline & L-389 & & & 0.23 & 0.30 & 0.35 & 0.44 & $<0.05$ & $<0.08$ & $<0.05$ & $<0.5$ & & & & & 0.18 & & 0.10 & & $<0.05$ & \\
\hline & L-390 & 0.37 & 9.1 & 0.17 & 0.23 & 0.40 & 0.52 & $<0.06$ & $<0.08$ & $<0.05$ & $<0.5$ & 0.044 & & & & 0.30 & & 0.10 & & $<0.05$ & \\
\hline
\end{tabular}


Table 10 (contd)

\begin{tabular}{|c|c|c|c|c|c|c|c|c|c|c|c|c|c|c|c|c|c|c|c|c|c|}
\hline \multirow[b]{2}{*}{ Sample } & \multirow{2}{*}{$\begin{array}{l}\text { Sample } \\
\text { No. }\end{array}$} & \multicolumn{2}{|c|}{$\mathrm{C}_{\mathrm{B}}$} & \multicolumn{2}{|c|}{$\mathrm{Ca}$} & \multicolumn{2}{|c|}{$\mathrm{Ba}$} & \multicolumn{2}{|c|}{ A1 } & \multicolumn{2}{|c|}{ Mo } & \multicolumn{2}{|c|}{$s_{r}^{c}$} & \multicolumn{2}{|c|}{$\mathbf{T i}$} & \multicolumn{2}{|c|}{ Si } & \multicolumn{2}{|c|}{$\mathrm{Ni}$} & \multicolumn{2}{|c|}{$\mathrm{Fe}$} \\
\hline & & $\mathrm{mg} / \mathrm{L}$ & (NL) & $\mathrm{mg} / \mathrm{L}$ & (NL) & $\mathrm{mg} / \mathrm{L}$ & (NL) & $\mathrm{mg} / \mathrm{L}$ & (NL) & $\mathrm{mg} / \mathrm{L}$ & (NL) & $\mathrm{mg} / \mathrm{L}$ & (NL) & $\mathrm{mg} / \mathrm{L}$ & (NL) & $\mathrm{mg} / \mathrm{L}$ & (NL) & $\mathrm{mg} / \mathrm{L}$ & (NL) & $\mathrm{mg} / \mathrm{L}$ & (NL) \\
\hline $\mathrm{XB9} 9-3$ & $L-391$ & 0.33 & 8.0 & 0.16 & 0.21 & 0.32 & 0.41 & $<0.05$ & $<0.08$ & $<0.05$ & $<0.5$ & & & & & 0.28 & & 0.08 & & 0.08 & \\
\hline & $\begin{array}{l}\mathrm{L}-392 \\
\mathrm{~L}-393 \\
\mathrm{~L}-394^{\mathrm{i}}\end{array}$ & $\begin{array}{c}\text { ND } \\
? .05\end{array}$ & $\begin{array}{r}\text { ND } \\
69.6\end{array}$ & $\begin{array}{l}1.87 \\
\text { AI } P^{k}\end{array}$ & & 3.61 & & 0.59 & & 0.15 & & & & & & 2.41 & 145 & 0.46 & 21.7 & 0.12 & 1.6 \\
\hline $\begin{array}{l}\text { Neutron } \\
\text { activated }\end{array}$ & $\mathrm{L}-395$ & & 40 & & & & & & & & & & & & & & & & & & \\
\hline $\begin{array}{l}\text { XB93 } \\
\text { T }-3 / 10 / 81,22: 95\end{array}$ & $\begin{array}{l}\mathrm{L}-396 \\
\mathrm{~L}-397 \mathrm{j}\end{array}$ & & 51 & & & & & & & & & & & & & & & & & & \\
\hline XB93-1500-4 & $L-398$ & 1.23 & 25.8 & & & 1.43 & 1.57 & 0.81 & 1.11 & 0.07 & 0.57 & & & & & 0.85 & & 0.57 & & 0.14 & \\
\hline XB93-1500-5 & $\bar{L}-399$ & 1.18 & 27.9 & & & 1.01 & 1.25 & 0.72 & 1.11 & 0.09 & 0.82 & & & & & 0.82 & & 0.60 & & 0.11 & \\
\hline XB93-1500-6 & $L-400$ & 1.22 & 28.5 & & & 1.00 & 1.23 & 0.70 & 1.07 & 0.07 & 0.63 & & & & & 0.86 & & 0.57 & & 0.13 & \\
\hline XB93-500-1 & L-401 & 1.08 & 26.7 & & & 1.19 & 1.54 & 0.80 & 1.29 & 0.08 & 0.77 & & & & & 0.74 & & 0.54 & & 0.11 & \\
\hline XB93-500-2 & $L-402$ & 1.11 & 26.4 & & & 1.26 & 1.57 & 0.74 & 1.14 & 0.07 & 0.64 & & & & & 0.80 & & 0.55 & & 0.13 & \\
\hline X893-500-3) & $\mathrm{L}-403$ & 0.10 & 2.4 & & & 0.27 & 0.33 & 0.20 & 0.31 & $<0.05$ & $<0.5$ & & & & & 0.14 & & 0.28 & & 0.10 & \\
\hline IAEA & $L-404$ & 0.11 & & & & 0.12 & 0.16 & 0.06 & 0.10 & $<0.05$ & $<0.5$ & & & & & 0.10 & & $<0.05$ & & $<0.05$ & \\
\hline Procedure & $L-405$ & 0.11 & 2.4 & & & 0.09 & 0.11 & 0.09 & 0.14 & $<0.05$ & $<0.5$ & & & & & 0.15 & & $<0.05$ & & $<0.5$ & \\
\hline Blank (no mesh) & $L-406$ & & & & & & & & & & & & & & & & & & & & \\
\hline Blank ${ }^{1}$ (me日h) & $L-407$ & 1.07 & 26.3 & & & 1.20 & 1.55 & 0.71 & 1.14 & 0.08 & 0.76 & & & & & 0.83 & & 0.60 & & 0.09 & \\
\hline XB93-1500-1 & $L-408$ & 1.32 & & & & 1.53 & 1.64 & 0.94 & 1.25 & 0.08 & 0.63 & & & & & 1.08 & & 0.71 & & 0.18 & \\
\hline XB93-1500-2 & $L-409$ & 0.08 & & & & 0.24 & 0.33 & 0.20 & 0.34 & $<0.05$ & $<0.5$ & & & & & 0.13 & & 0.25 & & 0.07 & \\
\hline XB93-1500-3)k & $\mathrm{L}-410$ & 0.14 & & & & 0.14 & 0.19 & 0.05 & $<0.08$ & $<0.05$ & $<0.5$ & & & & & 0.12 & & $<0.05$ & & $<0.05$ & \\
\hline IAEA & $\mathrm{L}-411$ & 0.14 & & & & 0.11 & 0.14 & 0.07 & 0.11 & $<0.05$ & $<0.5$ & & & & & 0.15 & & $<0.05$ & & 0.06 & \\
\hline Procedure & $L-412$ & & & & & & & & & & & & & & & & & & & & \\
\hline
\end{tabular}


Table 10 (contd)

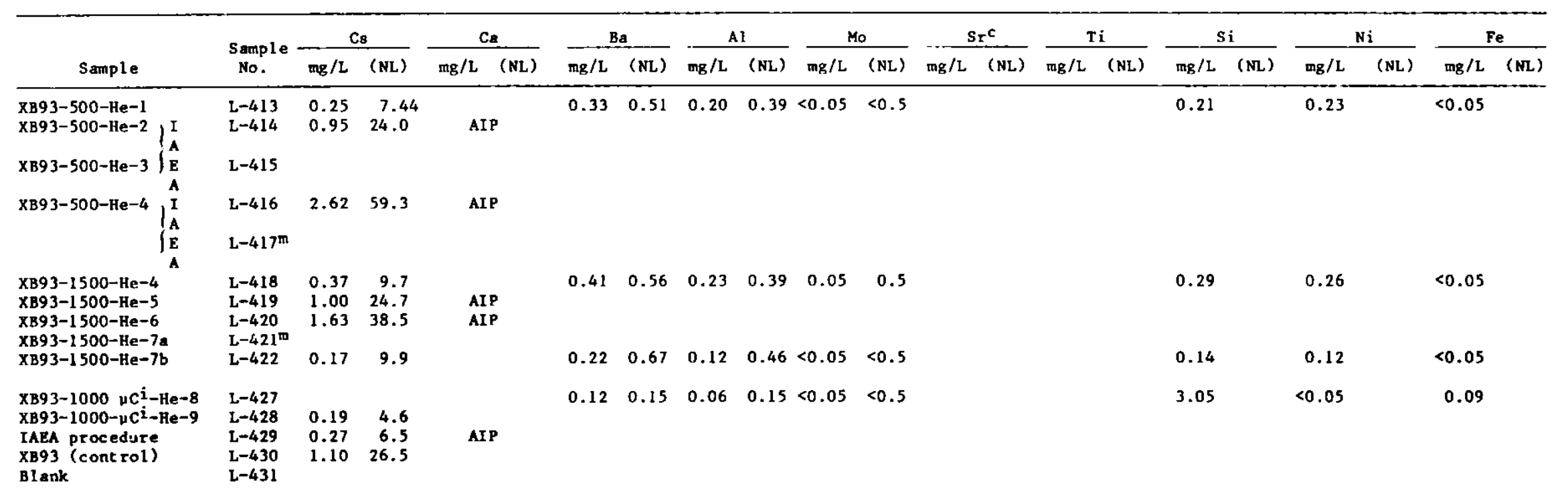

\section{(contd)}


Table 10 (contd)

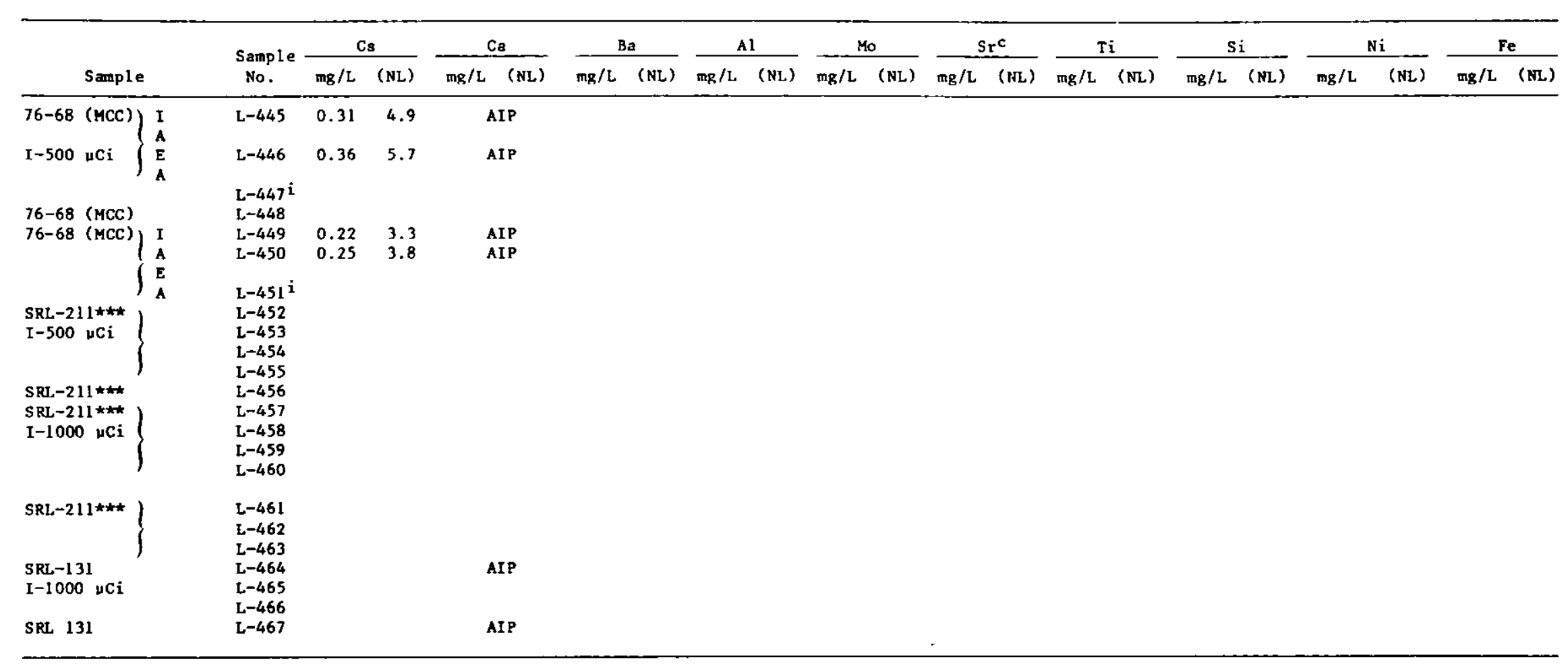


Table 10 (contd)

(NL) $i$ values are calculated using the composition of $X 8393$ as reported in Table 11

$b_{D F}=$ deionized water. $S W=s i l$ icate water. $B=b r i n e$.

corrected for blank.

NS - No solution.

enD - Not determined.

f Leachate was supposed to be SW but was actually DIW.

${ }^{8}$ Leachate was supposed to be B but was actually DIW.

haIP - Analysis in progress.

$i_{\text {Test }}$ completed $7 / 2$.

$\mathbf{j}_{\text {Test aborted. }}$

${ }^{k}(N L)_{i}$ values are cumulative for sequential time periods.

leachate spilled in preparation.

"Test to be completed $7 / 10$.

"NA - Not analyzed. 
Table 11. Solid SYNROC Sample Analyses

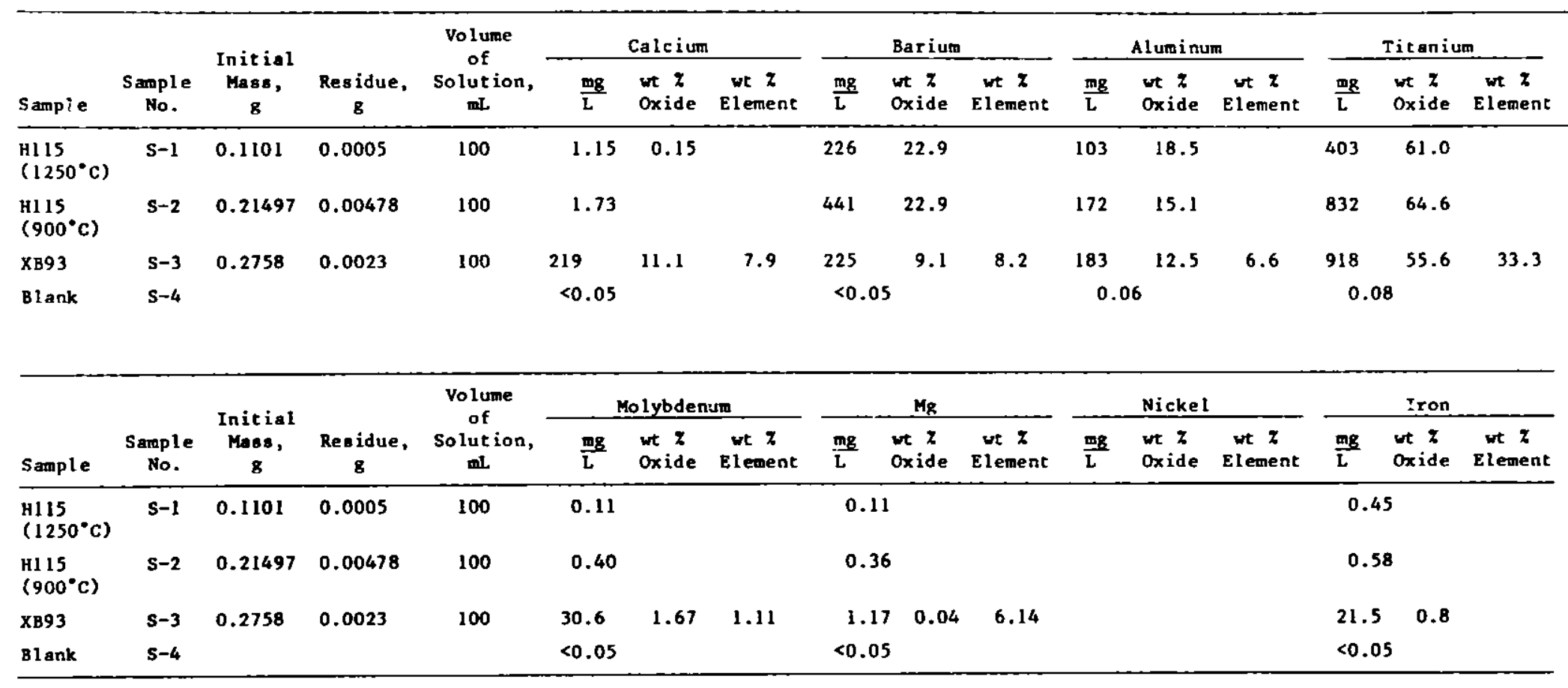


Activated Samples

$$
(\mathrm{NL})_{i}=\frac{A_{i}}{A_{0}} \times \frac{\text { Vol Efft }}{S A} \times M
$$

where $A_{i}$ is the activity of element " $i$ " in the leachate at $T_{0}$

$A_{0}$ is the activity of element " $i$ " in the solid at $T_{0}$

$\mathrm{SA}$ is the surface area $\left(\mathrm{m}^{2}\right)$ of the sample

$M$ is the original mass of the sample

Vol Efft is the inverse fraction of the leachate used for analysis

No conclusions concerning leachate trends will be made until all analyses have been received; however, the following points may affect interpretation results:

(1) Leach tests (except 377-382, 384-391, 404, 405, 411, and 412 , done in polypropylene bottles) were done in teflon containers by MCC-1 or IAEA procedures. There seems to be a definite effect whereby the leach results in polypropylene bottles are one-third those in teflon. This is best observed by comparing the results for tests L-380, L-381, L-382, L-387, and L-390 (which are replicates) with tests $\mathrm{L}-383$ and 430 (which are replicates). Analysis of the results of two further tests should settle the issue.

(2) Tests at $200^{\circ} \mathrm{C}$ were done in two different types of teflon containers. Although no effect of type of teflon is expected, it cannot be ruled out.

(3) NAA of SYNROC leachates could detect only $134 \mathrm{Cs}, 131_{\mathrm{Ba}}$, and ${ }^{141} \mathrm{Ce}$. This is because the large quantity of $134 \mathrm{Cs}$ in the leachate creates a high background, masking the other elements.

(4) NAA of SYNROC samples may cause the leach rate of $134 \mathrm{Cs}$ to increase five to seven times while not affecting the leach rate of $131_{\mathrm{Ba}}$.

Points 3 and 4 should be considered in future work.

b. Weathering Studies

Weathering studies designed to produce weathered (hydrated) glass samples are cont inuing. Samples of PNL 76-68 glass, SRL frit 211 , and SRL frit $211^{\star}$ are being weathered at $80^{\circ} \mathrm{C}$ and $90 \%$ relative humidity. The weathered samples will allow description of the hydration process and, when the glass has been subjected to leaching conditions, will be used to evaluate the durability of hydrated glass. 
III. BRITTLE FRACTURE STUDIES

(L. J. Jardine, W. J. Mecham, and G. T. Reedy)

\section{A. Introduction}

A property of solid radioactive waste forms that must be known is their resistance to dispersion from mechanical impacts received during normal and accidental conditions of processing, handling, interim storage, and transportation. A brittle fracture methodology is being developed which relates the impact-stress distribution to the lognormal size distribution of the particles generated by impact fracture. The practical application of this fracture mode 1 is to predict the total fracture-surface area and the mass of particles of respirable sizes produced by impact fractures.

Development and preliminary verification of this model has been provided by laboratory-scale tests of impacts of reference glass specimens and by analyses of data available in the technical literature on (1) impacts of various brittle materials and (2) impacts of large-scale simulated wasteglass canlsters.

Examination of the validity and utility of this brittle-fracture methodology is being made for a range of practical impact conditions and for various solid waste materials. It is anticipated that the experimental results will establish correlations with impact energy of (1) lognormal size-distribution parameters and (2) fracture surface areas, as well as the effects on particle size and surface area of material propertles of brittle-waste packages having various sizes and configurations.

B. Comparative Impact Testing of Alternative High Level Waste Forms: Generation of Respirable Sizes

1. Introduction

Impact test data of solid alternative waste forms are needed, in conjunction with other characterization measurements, to assess the probable and/or worst-case performance of waste forms during handling, transportation, and storage conditions. The two major measurable responses useful to characterize the results of mechanical impacts of brittle waste forms are (1) the particle size distribution of the impact fracture fragments, including the quantity of particles which could become afrborne or respirable and (2) the increase in surface area caused by the generation of impact fracture fragments. The particle size distribution can be used to assess source terms for airborne releases, and the surface area increase can be used to assess the increase in source terms for leach rates.

A brittle fracture study at Argonne National Laboratory (ANL) 18 currently developing the experimental characterization methodologies required to assess the above two major responses to impacts for large canisters of glass waste forms. The ANL study utilizes modeling in confunction with closely coupled laboratory-scale experimental verification to establish engineering correlations, and eventually to predict the se two measurable responses. As a consequence of these Impact testing and characterization measurements, detailed procedures for impacting waste forms and for measuring particle size distributions of fracture particulates have been developed. 
Because of a current, immediate need for comparative impact response data for the alternative waste forms under development in the High Level Waste Technology Program and the lack of standard impact test procedures (i.e., MCC-10) from the Materlals Characterization Center at Battelle Pacific Northwest Laboratory, standardized sets of impact test criteria and characterization procedures have been defined and used to measure the respirable sizes (arbitrarily defined as all particles sizes $\leq 10 \mu_{\mathrm{m}}$ in these test comparisons) for the currently avallable alternative waste forms.

This report summarizes the results of standardized drop-weight Impact test and characterization procedures used to generate, collect, and measure the respirable sizes generated in the impact testing of alternative waste forms. The seven simulated alternative waste forms impacted were (1) SRL frit 131 borosilicate glass, (2) ORNL FUETAP concrete, (3) LLL SYNROC ceramic (4) Rockwell TAILORED CERAMIC, (5) Catholic University (Cath. U) high-silica glass, (6) PNL 76-68 glass, and (7) ANL-MSD SYNROC. Preliminary test results produced varlations in the measured respirable fractions of a factor of only seven between the "best" and "worst" waste forms.

The measurement of increases in surface area produced by fracture is not addressed in this seport.

\section{Experimental Procedure}

A standardized set of drop-weight impact test conditions and characterization procedures were defined and applied to seven generic types of alternative high-level waste forms to characterize the resulting respirable sizes of the fracture particulates. To allow additional comparison, Pyrex glass and coal were also tested. The test procedures are believed to be suitable for consider=tion as a standard MCC-10 dynamic impact test for brittle waste forms.

For all tests, cylindrical samples of similar size (about $2.5 \mathrm{~cm}$ by $2.5 \mathrm{~cm}$ ) we re impacted by dropping a steel bar of about $9.9 \mathrm{~kg}$ from a predetermined height to provide a maximum input kinetic energy of $10 \mathrm{~J} / \mathrm{cm}^{3}$ of specimen volume. The specimens were all impacted on their sides within a closed impact chamber where particles were collected. The chamber, provided with a bellows to transmit the impact force, is described in the report for the previous quarter [STEINDLER-1981C].

The complete size distributions of the particles resulting from fracture were measured in a Sonic Sifter sieving device (for particles larger than $90 \mu \mathrm{m}$ ) and in a Coulter counter (for particles whose size was between about $5 \mu \mathrm{m}$ and $90 \mu \mathrm{m})$. Size fractions based on the total mass of the specimens were plotted on lognormal graphical coordinates, and the values of the lognormal size parameters (the mass median, $D_{g}$, and the geometric standard deviation, $\sigma_{g}$ ) we re calculated by linear regression of the measured size data. The particles of respirable size were determined as the cumulative mass fraction of particles smaller than $10 \mu \mathrm{m}$ (as a weight percent of the specimen mass). 


\section{Preparation of Test Spectmens}

Cylindrical specimens we core-drilled from available bulk material in all but three cases (SYNKOC MSD, FUETAP, and PYREX, the last of which is a reference material), which were available in cylindrical form of the required diameter. The ends of the specimens were flat. All glass specimens were annealed, but the others not.

\section{Description of Materials Tested}

The results for materials tested in this report period are compared with results reported in [STEINDLER-1981C]. Plots of the particle-size distribution data for seven new tests are reported here. The plots of the other tests were given in the previous quarterly report [STEINDLER-1981C]. The chemical compositions of simulated waste materials are summarized in Table 12.

\section{a. FRIT 131 Glass}

This is a simulated borosilicate waste glass supplied by Savannah River Laboratory (SRL). The glass contained no urantum, but was loaded with about 28 wt \% simulated SRL TDS calcine. The glass was supplied by SRL from their pilot 1/3-scale continuous melter. Specimens for impact testing were prepared by remelting the SRL frit 131 glass in stainless steel crucibles at $1100^{\circ} \mathrm{C}$ for about $1 / 2 \mathrm{~h}$ under a nitrogen purge, annealing the glass at $550^{\circ} \mathrm{C}$ for several hours, and then core-drilling specimens $25.5 \mathrm{~mm}$ ob by $27 \mathrm{~mm}$ long. The composition (presented $1 \mathrm{n}$ (STEINDLER-1981C]) is repeated in Table 12 .

One test of this material was reported in [STEINDLEK-1981C]. A second specimen, SRL 131 glass (2), was tested during the present report period, and the particle size distribution is plotted in Fig. 10. The principal fracture result, the respirable size fraction at $D=10^{-5} \mathrm{~m}$ or $10 \mu \mathrm{m}$, is evident from the plot, and the quantitative results for all the tests are presented and may be compared in Table 13, along with earlier reported results. The respirable fractions for the specimen frit 131 glass (2) and for the previously made test frit 131 glass (1) agree. A more complete comparison of results is given later. The straight line shown in Fig. 10 represents the best statistical size distribution of the actual fracture particulate, for the full range of particle sizes (smaller than $2 \mathrm{~mm}$ ). The significance of the departures of data points from the straight line is discussed below in Section III.C.2.

\section{b. PNL 76-68 Glass}

This is a simulated waste glass produced by Pacific Northwest Laboratory (PNL) for the Materlals Characterization Center (MCC) "round robin" tests. A supply was obtalued from PNL, and a bulk piece was prepared for core drilling by remelting. Tests results are shown in Fig. 11 and Table 13 . They agree closely with those for the SRL frit 131 glass specimens.

\section{c. SYNROC MSD}

Two cylindrical specimens of SYNROC, each containing 10 wt \% PW-4b [MENDEL] simulated waste, were obtained from the Materials Science Division (MSU) of ANL. The specimens were made by cold-pressing and sintering 
Table 12. Nominal Compositions of Alternative Waste Forms Used In this Comparative Impact Study

\begin{tabular}{|c|c|c|c|c|c|c|c|}
\hline \multirow{4}{*}{$\begin{array}{l}\text { Component } \\
\text { "Ma Lrix" }\end{array}$} & \multicolumn{5}{|c|}{ Waste Form } & \multirow{3}{*}{$\begin{array}{c}\text { High-Silica } \\
\text { Glass }\end{array}$} & \multirow[b]{3}{*}{ Pyrex } \\
\hline & \multirow{2}{*}{$\begin{array}{c}\text { SRL } 131 \\
\text { Glass } \\
\end{array}$} & \multicolumn{2}{|c|}{ SYNROC } & \multirow{2}{*}{$\begin{array}{l}\text { TAILORED } \\
\text { CERAMIC } \\
\end{array}$} & \multirow{2}{*}{$\begin{array}{c}\text { FUETAP } \\
\text { Concrete }\end{array}$} & & \\
\hline & & "B" & "D" & & & & \\
\hline & \multicolumn{7}{|c|}{ Weight percent of component } \\
\hline $\mathrm{SiO}_{2}$ & 41.7 & & & & $27.75 a$ & 54.9 & 81 \\
\hline $\mathrm{B}_{2} \mathrm{O}_{3}$ & 10.6 & & & & & 3.3 & 13 \\
\hline $\mathrm{TiO}_{2}$ & 0.7 & 59.4 & 18.7 & 2.0 & & & - \\
\hline $\mathrm{Al}_{2} \mathrm{O}_{3}$ & & 6.5 & 1.0 & 5.8 & & 8.0 & 2 \\
\hline $\mathrm{Li}_{2} \mathrm{O}_{3}$ & 4.1 & & & & & & - \\
\hline $\mathrm{Na}_{2} \mathrm{O}$ & 12.7 & & & & & & 4 \\
\hline $\mathrm{Cs}_{2} \mathrm{O}$ & & & 0.3 & & & 0.1 & \\
\hline $\mathrm{MgO}$ & 1.4 & & & & & & \\
\hline $\mathrm{CaO}$ & & 15.2 & 3.4 & & & & 1 \\
\hline $\mathrm{BaO}$ & & 8.0 & 1.4 & & & & \\
\hline $\mathrm{Zro}$ & 0.4 & 10.9 & 4.9 & & & 0.01 & \\
\hline $\mathrm{La}_{2} \mathrm{O}_{3}$ & $0 . \dot{4}$ & & & 7.2 & & & \\
\hline Flyash & & & & & 11 & & \\
\hline Cement, type 1 & & & & & & & \\
\hline Wa ter & & & & & $18^{\mathrm{b}}$ & & \\
\hline Water Reducer & & & & & $1.25^{\mathrm{b}}$ & & \\
\hline "Natrix" Totals & 72 & 100 & 32.8 & 15 & 80 & 66.3 & 100 \\
\hline \multicolumn{8}{|l|}{$\begin{array}{c}\text { SRL } \\
\text { "Waste" }\end{array}$} \\
\hline $\mathrm{Fe}_{2} \mathrm{O}_{3}$ & 13.9 & - & 24.2 & 12.6 & 9.4 & $14.5^{\mathrm{C}}$ & \\
\hline $\mathrm{Al}_{2} \mathrm{O}_{3}$ & 3.0 & - & 18.9 & $46.2^{d}$ & 1.8 & & \\
\hline $\mathrm{MnO}_{2}$ & 3.7 & - & 7.5 & 10.4 & 2.6 & 4.3 & \\
\hline $\mathrm{CaO}$ & 1.0 & - & 1.3 & 0.8 & 0.5 & 0.6 & \\
\hline $\mathrm{NiO}$ & 1.6 & - & 3.1 & 1.8 & 1.2 & 1.9 & \\
\hline $\mathrm{SiO}_{2}$ & & - & 0.5 & 1.4 & 0.2 & & \\
\hline $\mathrm{Na}_{2} \mathrm{O}$ & 0.6 & - & 3.4 & 4.6 & 1.3 & 2.0 & \\
\hline $\mathrm{Na}_{2} \mathrm{SO}_{4}$ & 0.4 & - & 0.6 & 0.4 & 0.2 & & \\
\hline sro & & - & 0.4 & & 0.2 & 0.1 & \\
\hline $\mathrm{CeO}_{2}$ & & - & 0.4 & & 0.4 & 1.1 & \\
\hline $\mathrm{Gd}_{2} \mathrm{O}_{3}$ & & - & 0.4 & & 0.4 & & \\
\hline $\mathrm{Nd}_{2} \mathrm{O}_{3}$ & & - & 0.4 & & & 0.4 & \\
\hline $\mathrm{Eu}_{2} \mathrm{O}_{3}$ & & - & 0.4 & & & & \\
\hline $\mathrm{UO}_{2}$ & & - & 2.1 & d & & & \\
\hline Zeolite & 2.7 & - & - & 7.3 & 1.6 & & \\
\hline $\mathrm{P}_{2} \mathrm{O}_{5}$ & - & - & - & - & - & 5.0 & \\
\hline
\end{tabular}

aUETAP used Ot tawa sand, which was placed 1 in $\mathrm{S}_{1} \mathrm{O}_{2}$ column.

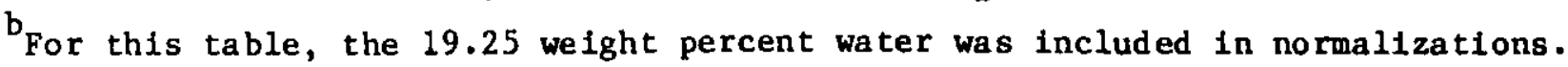

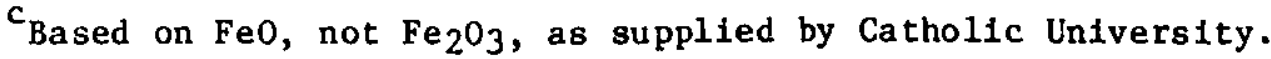

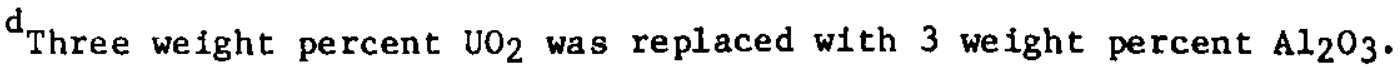


Table 13. Respirable Particles Generated in Comparative Impact Tests ${ }^{a}$

\begin{tabular}{|c|c|c|c|c|c|c|c|c|c|}
\hline \multirow[b]{3}{*}{ Material } & \multirow[b]{3}{*}{$\begin{array}{c}\text { Source } \\
\text { Laboratory }\end{array}$} & \multirow{2}{*}{\multicolumn{3}{|c|}{$\begin{array}{c}\text { Size of Cylindrical } \\
\text { Specimen Impacted }\end{array}$}} & \multicolumn{2}{|c|}{$\begin{array}{l}\text { Input Energy } \\
\text { of Impact }\end{array}$} & \multirow{2}{*}{\multicolumn{2}{|c|}{$\begin{array}{c}\text { Particle-Size } \\
\text { Distribution: } \\
\text { Lognormal Parameters }\end{array}$}} & \multirow{3}{*}{$\begin{array}{c}\text { Fraction of } \\
\text { Fracture Particles, } \\
\text { of Kespirable Size, } \\
\text { wt } \%\end{array}$} \\
\hline & & & & & \multirow{2}{*}{$\begin{array}{c}\text { Total } \\
\text { Energy, } \\
\mathrm{J}\end{array}$} & \multirow{2}{*}{$\begin{array}{l}\text { Energy } \\
\text { Density } \\
\mathrm{J} / \mathrm{cm}^{3}\end{array}$} & & & \\
\hline & & $\underset{\mathrm{mm}}{\mathrm{Dia}}$ & $\begin{array}{c}\text { Length, } \\
\mathrm{mm}\end{array}$ & $\begin{array}{c}\text { Mass, } \\
8 \\
\end{array}$ & & & $\begin{array}{l}\mathrm{D}_{\mathrm{g}}, \\
\text { min }\end{array}$ & $\sigma_{g}$ & \\
\hline SRL Frit 131 glass (1) & SRL & 25.5 & 27 & 39.7 & 146 & 10 & $2.6 \pm 0.4$ & $6.4 \pm 0.2$ & $0.14 \pm 0.02$ \\
\hline SRL Frit 131 glass (2) & SRL & 25.4 & 20.1 & 40.7 & 148 & 10 & $2.6 \pm 0.2$ & $6.6 \pm 0.4$ & $0.18 \pm 0.05$ \\
\hline PNL 76-68 glass & PNL & 25.4 & 25.2 & 37.7 & 128 & 10 & $2.7 \pm 0.3$ & $6.9 \pm 0.1$ & $0.18 \pm 0.01$ \\
\hline SYNROC MSD (1) & ANL & 20.7 & 20.4 & 29.9 & 69 & 10 & $6.3 \pm 2.1$ & $8.6 \pm 0.6$ & $0.14 \pm 0.03$ \\
\hline SYNROC MSD (2) & ANL & 20.7 & 19.9 & 28.4 & 67 & 10 & $7.1 \pm 3.3$ & $8.3 \pm 0.7$ & $0.10 \pm 0.03$ \\
\hline SYNROC B & LLLL & 26.8 & 25.9 & 60.5 & 146 & 10 & $4.2 \pm 0.8$ & $7.6 \pm 0.3$ & $0.15 \pm 0.02$ \\
\hline SYNROC D & LLLL & 25.4 & 27.3 & 53.5 & 138 & 10 & $4.7 \pm 0.7$ & $8.1 \pm 0.3$ & $0.16 \pm 0.02$ \\
\hline High-silica glass & Cath U & 28.1 & 28.9 & 47.2 & 178 & 10 & $2.7 \pm 0.6$ & $7.2 \pm 0.3$ & $0.22 \pm 0.03$ \\
\hline Pyrex glass & ANL & 25.0 & 25.8 & 28.0 & 127 & 10 & $1.4 \pm 0.2$ & $6.0 \pm 0.2$ & $0.27 \pm 0.03$ \\
\hline Coal & ANL & 25.3 & 26.0 & 16.8 & 131 & 10 & $2.1 \pm 0.6$ & $6.3 \pm 0.3$ & $0.19 \pm 0.04$ \\
\hline TAILORED CERAMIC & Rockwe11 & 26.8 & 18.2 & 40.8 & 102 & 10 & $10 \pm 2$ & $8.3 \pm 0.3$ & $0.05 \pm 0.01$ \\
\hline FUETAP concrete & ORNL & 25.4 & 25.5 & 23.0 & 131 & 10 & $2.3 \pm 0.3$ & $7.9 \pm 0.2$ & $0.43 \pm 0.04$ \\
\hline
\end{tabular}

a Further description of materials and test conditions are given in the text. 


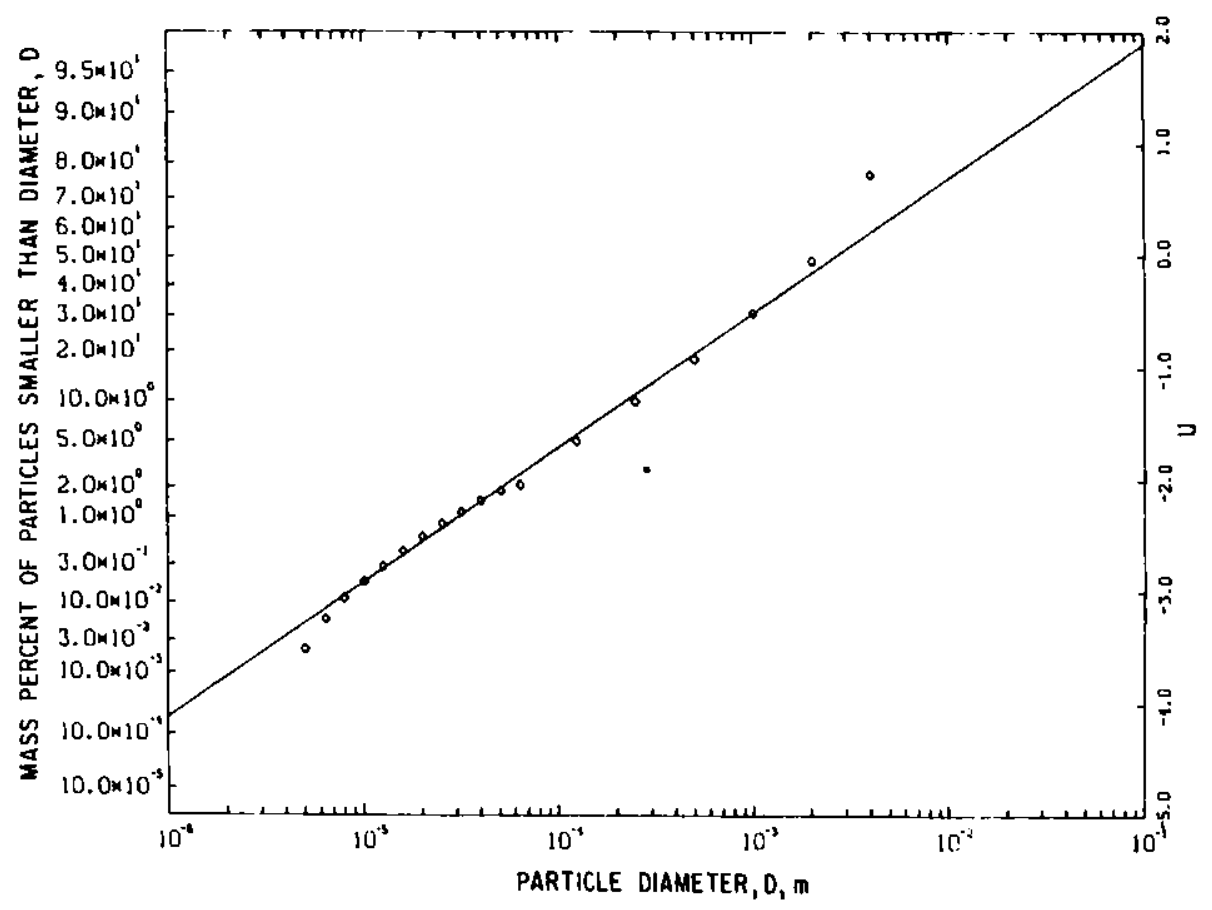

Fig. 10. Lognormal Plot of Cumulative Measured Weight Percent [P\%] vs. the Measured Particle Diameter or Mesh Size [D(m)] for SRL Frit 131 Glass (2) Impact Test. $10 \mathrm{~J} / \mathrm{cm}^{3}$.

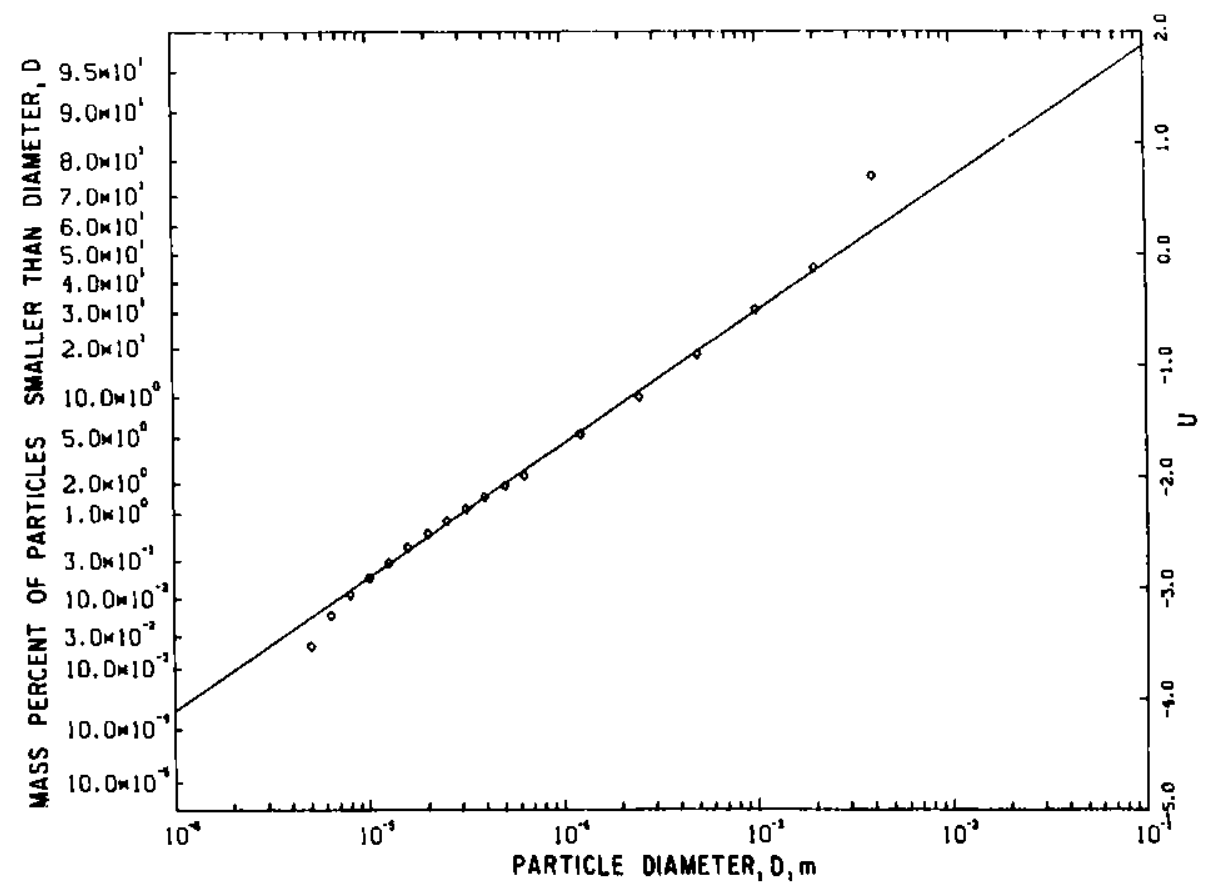

Fig. 11. Lognormal Plot of Cumulative Measured Weight Percent [P \%] vs. the Measured Particle Diameter or Mesh Size $[D(m)]$ for PNL 76-68 Glass Impact Test. $10 \mathrm{~J} / \mathrm{cm}^{3}$. 
at $1300^{\circ} \mathrm{C}$ for three hours in about $1 \mathrm{MPa}(10 \mathrm{~atm})$ of $\mathrm{CO} / \mathrm{CO}_{2}$. The density (calculated from geometry and mass) was $4.26 \mathrm{~g} / \mathrm{cm}^{3}$. Test results are shown in Figs. 12 and 13 and in Table 13. As shown in Table 13, these results are similar to those for the previously tested SYNROC B and SYNROC D, which are described below.

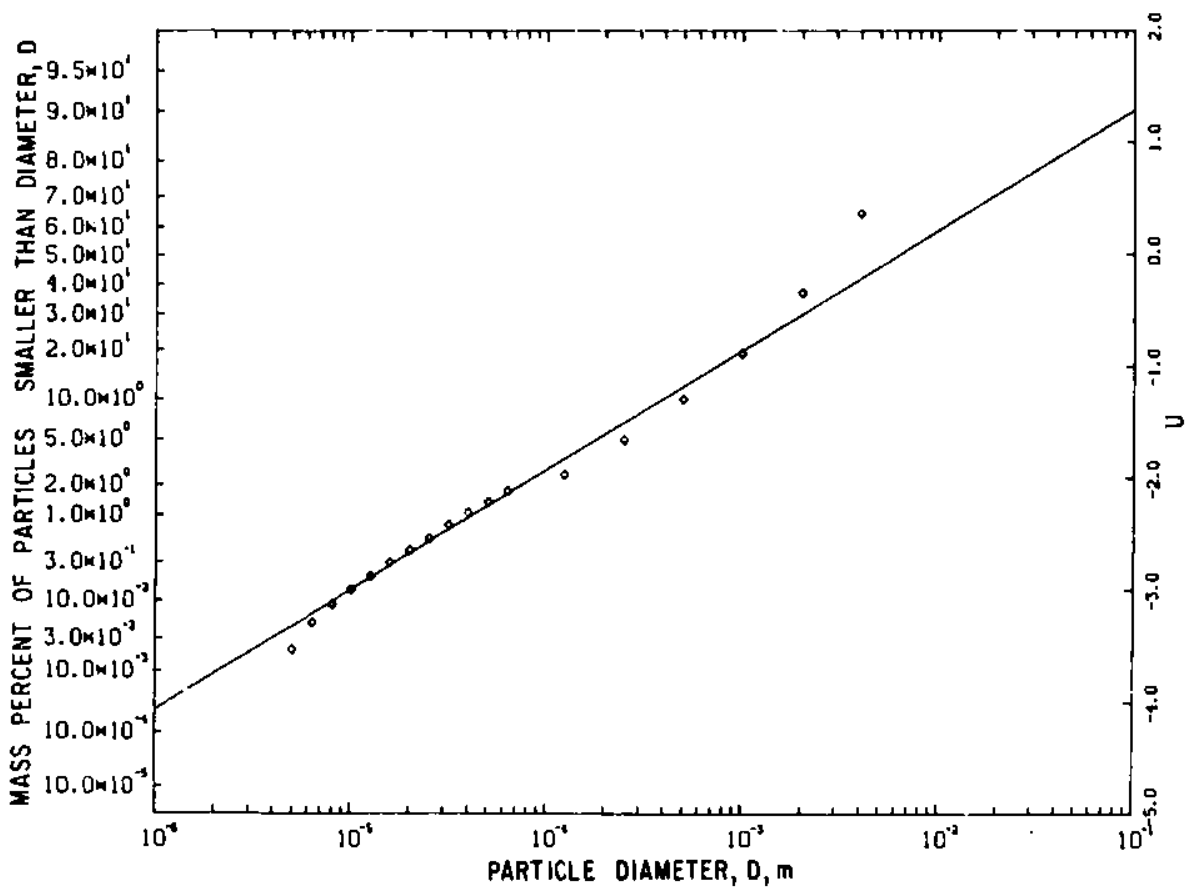

Fig. 12. Lognormal Plot of Cumulative Measured Weight Percent [P\%] vs. the Measured Particle Diameter or Mesh Size $[D(m)]$ for MSD SYNROC (1) Impact Test. $10 \mathrm{~J} / \mathrm{cm}^{3}$.

\section{d. SYNROC B and SYNROC D}

These are ceramic forms received from Lawrence Livermore Laboratory (LLL). SYNROC B ceramic contained no waste and was reported by LLL to have a density of $4.2 \mathrm{~g} / \mathrm{cm}^{3}$ (96\% T.D.). SYNROC D contained 64 wt \% waste, with a reported density of $3.96 \mathrm{~g} / \mathrm{cm}^{3}$ (92\% T.D.). Compositions are given in Table 12. Details of manufacture and sample preparation were given in [STEINDLER-1981C] along with graphs of the particle-size distributions. To allow comparison, previously published test results are included in Table 13.

\section{e. High-Silica Glass}

The composition of this simulated waste is given in Table 12 . The specimen, received from Catholic University (CU), had been core-drilled from a larger piece which had been formed by sintering powder in a crucible for $4 \mathrm{~h}$ at $1250^{\circ} \mathrm{C}$ in a vacuum. The as-received material was tested. It appeared to be heterogeneous (unlike glass), with zones of different colors. No analysis of the microstructure was available. Test results are shown in Fig. 14 and Table 13 . 


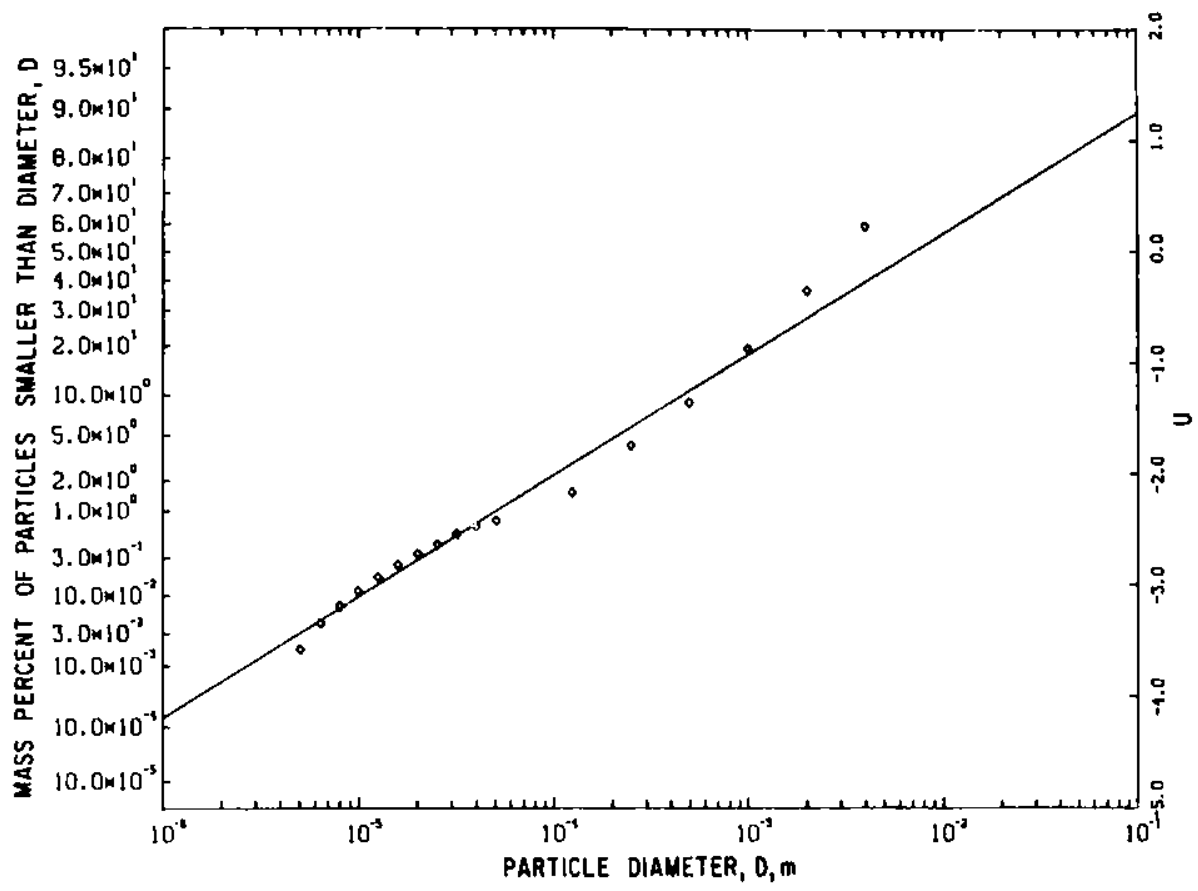

Fig. 13. Lognorma1 Plot of Cumulative Measured Weight Percent [P\%] vs. the Measured Particle Diameter or Mesh Size $[D(m)]$ for MSD SYNROC (2) Impact Test. $10 \mathrm{~J} / \mathrm{cm}^{3}$.

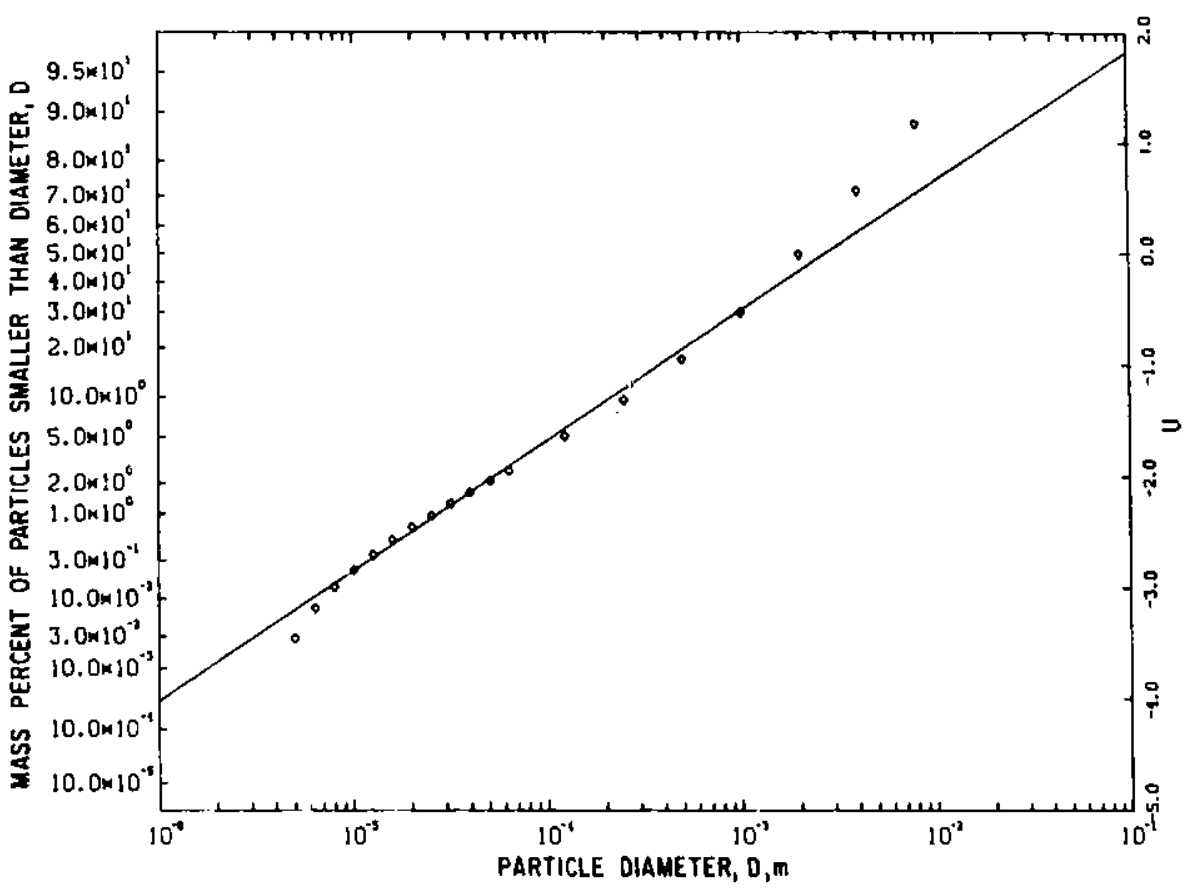

Fig. 14. Logn ,rmal Plot of Cumulative Measured Weight Percent [P\%] vs. the Measured Particle Diameter or Mesh Size $[D(m)]$ for CU High-Silica Impact Test. $10 \mathrm{~J} / \mathrm{cm}^{3}$. 


\section{f. Pyrex Glass}

This commercial glass contained no waste and was included only as a reference material. The specimen was cut from rod stock and annealed. Results are shown in Fig. 15 and Table 13.

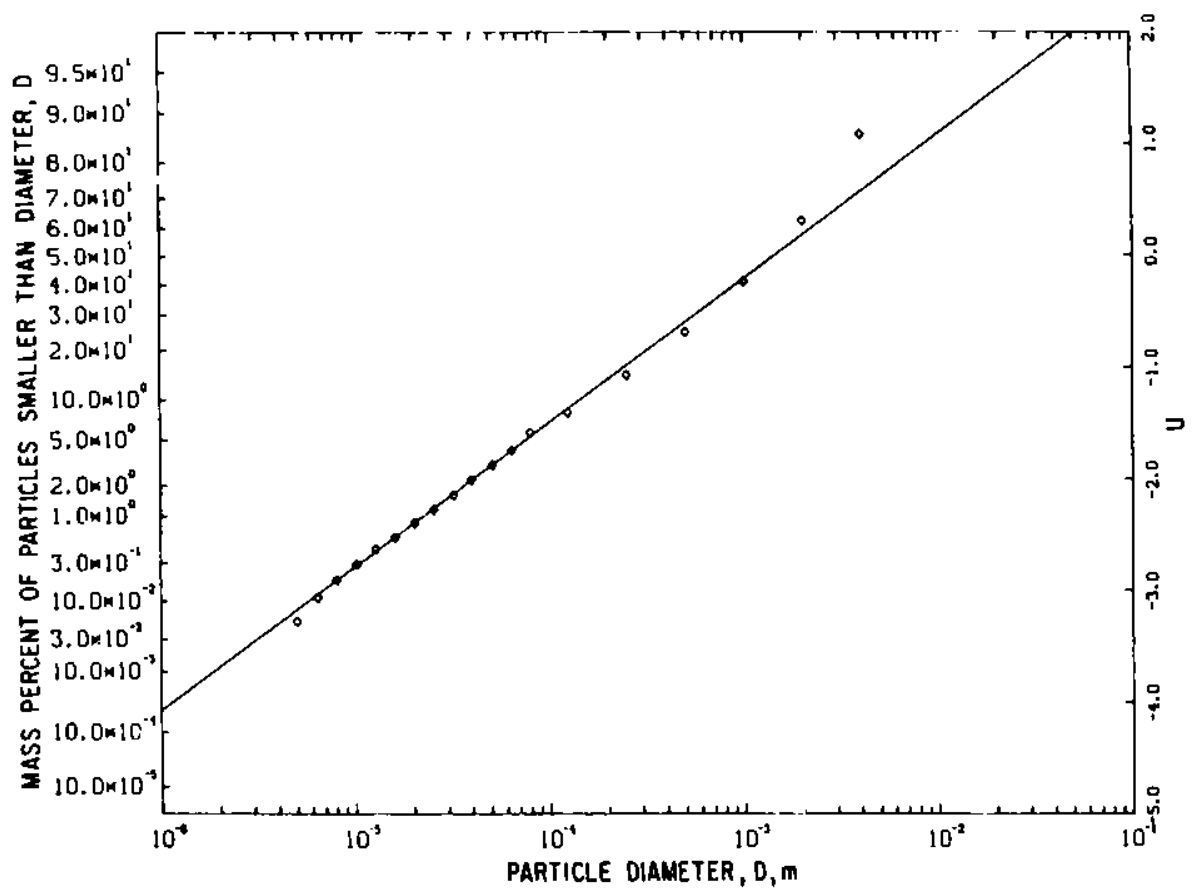

Fig. 15. Lognormal Plot of Cumulative Measured Weight Percent [P\%] vs. the Measured Particle Diameter or Mesh S1ze $[D(\mathrm{~m})]$ for Pyrex Impact Test. $10 \mathrm{~J} / \mathrm{cm}^{3}$.

\section{g. $\quad \underline{C o a 1}$}

To examine the possibility of using our test procedures for other research programs, a specimen of coal obtained from the ANl power plant stockpile was tested. Special care was necessary to obtain a sound specimen by core drilling. The particle size analysis shown in Fig. 16 and Table 13 is compareble to those of other materials tested.

\section{h. TAILORED CERAMIC}

Th1s hot-pressed ceramic was core-drilled from a bulk plece received from Rockwell International. The avallable size limited the length to that shown in Table 13. The previously reported composition is again given in Table 12; the graph of particle-size distribution and the details of manufacture are given in [STEINDLER-1981C]. The result of this test is included here to allow respirable fractions to be compared. The TAILORED CERAMIC showed the smallest respirable fraction of the materials tested. 


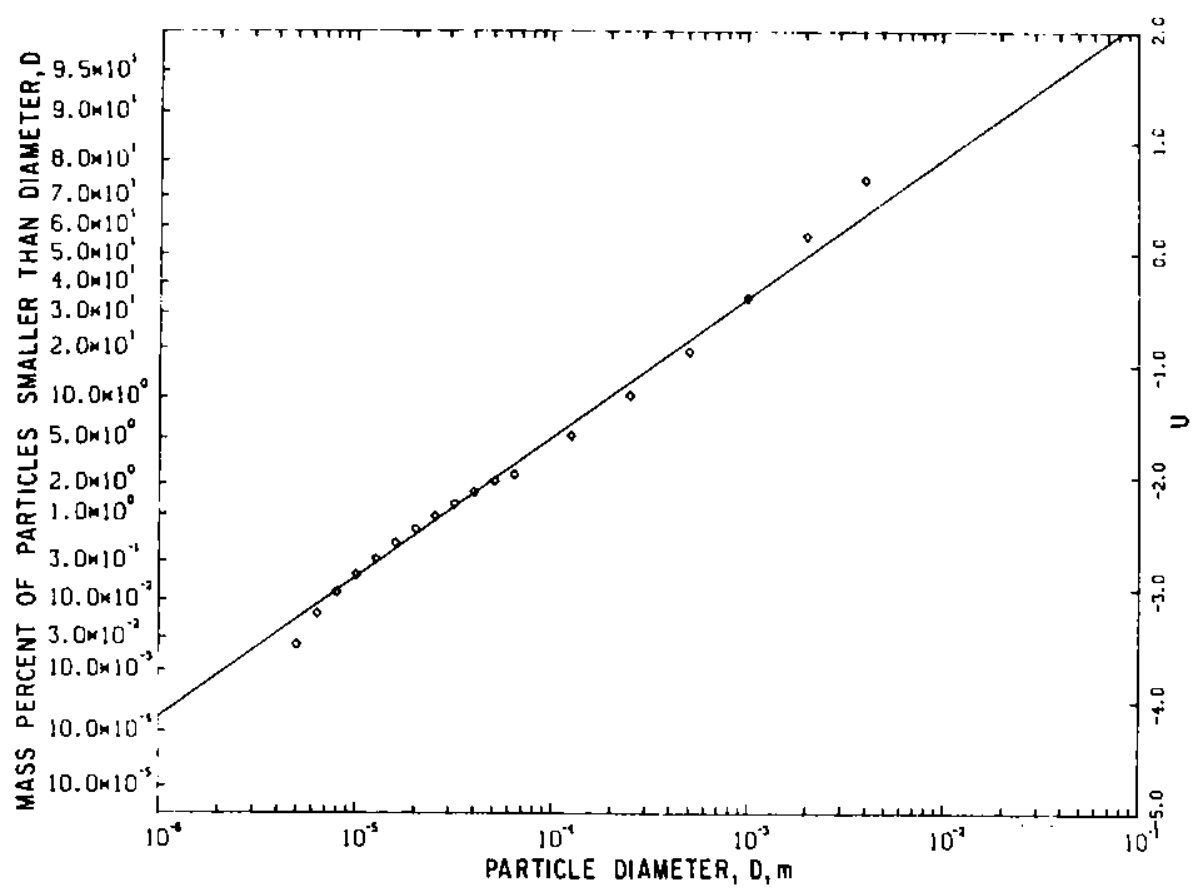

Fig. 16. Lognormal Plot of Cumulative Measured Weight Percent [P\%] vs. the Measured Particle Diameter or Mesh Size $[D(m)]$ for Coal Impact Test. $10 \mathrm{~J} / \mathrm{cm}^{3}$.

\section{i. FUETAP Concrete}

This is an Oak Ridge National Laboratory (ORNL) simulated waste concrete loaded with 20-25 wt \% simulated SRL waste; the concrete was dewatered at $250^{\circ} \mathrm{C}$. Cylindrical material was cut to size. Its composition (no uranium) is again presented in Table 12. Details of manufacture and a particle size plot are given in [STEINDLER-1981C]. Test results for one FUETAP lot shown in Table 13 are included to allow comparison.

\section{Discussion of Results}

The plots of empirical size data from sieving and Coulter counter analysis show lognornal size distribution in the range of $10 \mu \mathrm{m}$ to $2 \mathrm{~mm}$. It is presumed that this establishes lognormality for particles smaller than $10 \mu \mathrm{m}$ (which are not measurable with the present equipment). The particulates of 2-mm particle size and smaller contain $95 \%$ or more of the total surface area of a complete lognormal distribution (i.e., for diameters from zero to infinity) for particulates with the values of $\bar{D}_{g}$ and $\sigma_{g}$ listed in Table 13 . A comparison of particle size distributions is provided by Fig. 17, in which straight-line plots for all tests in Table 13 are shown. The plotted points are shown for each test particulate and correspond to (1) the cumulative mass fractions at diameter $10 \mu \mathrm{m},(2)$ the cumulative mass fraction for the particle diameters which corresponds to $50 \%$ of the cumulative surface area distribution and (3) the geometric mean diameters, $D_{g}$, defined for the mass fractions of $50 \%$. Measured surface areas are to be reported later. 


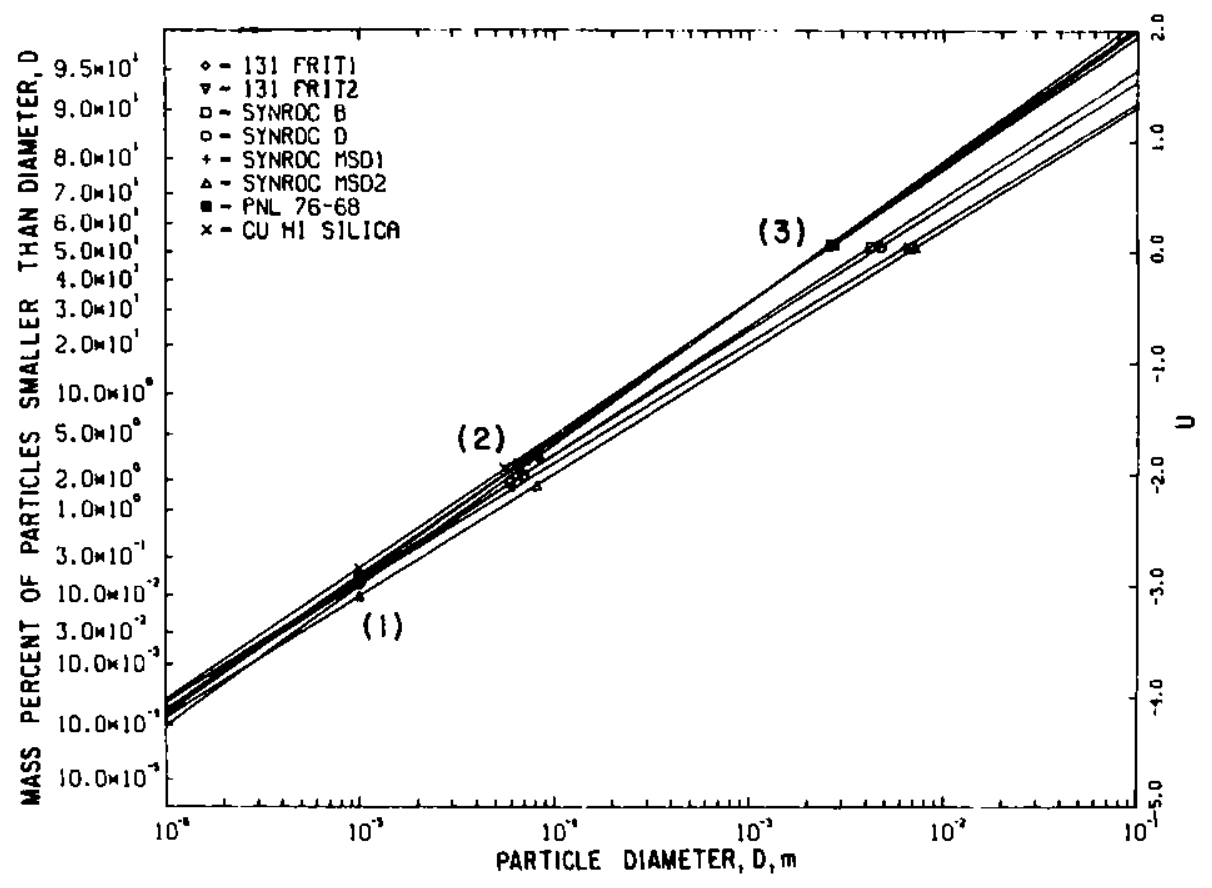

Fig. 17. Comparison of Lognormal Size Distributions for Eight Impact Tests at $10 \mathrm{~J} / \mathrm{cm}^{3}$ as Described in the Text

For all of the simulated waste glasses and ceranics except TAILORED CERAMIC, the values for respirable fraction agree within a factor of two. The highest respirable fraction of all is for FUETAP concrete. The lowest is that for TAILORED CERAMIC (Table 13).

In the two cases which show replication (Table 13), SRL frit 131 glass and SYNROC MSD, the results agree within the standard error of the regression analysis, for both the respirable fraction and the lognormal parameters. The LLL SYNKOC B and D specimens also are in good agreement.

As the data points on the plots of the particle-size distributions show (Figs. 10-16), there are departures from lognormality in Coulter counter (CC) data at about $5 \mu \mathrm{m}$ and $90 \mu \mathrm{m}$. This is an artifact of the method. At the lower limit of the CC range ( $5 \mu \mathrm{nl}$ for our instrument), particles are systematically undermeasured because of pulse electronics. 'Iherefore, extrapolation to smaller sizes is the more accurate representation of the actual fracture particulate. The departure from lognormality of CC data at its upper size limit $(90 \mu \mathrm{m})$ is also an artifact caused by the particular features of the wet sieving method used to prepare the CC sample. The particle diameter is determined differently by CC analysis and by sieving analysis, which contributes to the variations in the 80-100 $\mu \mathrm{m}$ size range. Overall, the large number of data points provide a reasonable statistical accuracy for the lognormal parameters, $D_{g}$ and $\sigma_{g}$, which, in turn, provide the best estinate of the actual size distribution of the fracture particulate. 


\section{Methodology Development \\ (W. J. Mecham) \\ 1. Background}

As part of the brittle fracture study, a comprehensive methodology is being developed to characterize the impact behavior of brittle materials under conditions of practical interest such as may be encountered in accidents during manufacture, handling, and transport of solid radioactive waste forms. One aim is to characterize the impact-resistance property of waste materials in terms of the increased environmental dispersability of the material as a consequence of impact fracture--namely, (1) the increased surface area generated for possible leaching by groundwater and (2) the amount of fracture particulate which is of respirable sizes (particles smaller than about 10- $\mu \mathrm{m}$ diameter). A second aim is to characterize impact severity (with respect to brittle fracture) in terms of body size and shape for practical collision velocities and other relevant factors.

In previous quarterly reports of this series (see title page), a review was presented of the technical literature, which showed the practical inadequacies of theoretical fracture mechanics and of experimental testing with respect to the above aims. The methods of continuum mechanics cannot deal either with discontinuities in the stress field nor with thermodynamically irreversible and time-dependent energy transformations. Reported experimental approaches relevant to the impact strength of materials have been largely confined to concerns with structural failure and brittle metals, rather than the extent of fracture of truly brittle materials such as glasses and ceramics.

The methodology discussed here is based on a combination of dimensional-analysis modeling principles, empirical energy-surface correlations for the crushing of minerals, lognormal particle statistics, and axisymmetric dynamic stress analysis. Methodology development has been described in quarterly progress reports of this series and in a topical report [MECHAM].

In this report, methodology on the following topics is updated: (a) evaluation of measurements of particle-size distributions, (b) evaluation of surface areas of fracture particulates, (c) energy and stress relations in impact compression, (d) stress distributions calculated from particle-size distributions, (e) energy partitioning in impact testing machines, and ( $f$ ) modeling of impact severities in free-fall impacts of waste-form prototypes.

2. Evaluation of Measurements of Size Distributions of Impact-Fracture Particulates

Size distributions of solid particulates are measured by separating the solid particulate sample into mass (or volume) increments according to particle size, i.e., the characteristic linear size, the particle diameter, $D$. For material of a given density, mass increments are equivalent to volume increments, $\Delta v_{j}$, for a given size range, $D_{j}-D_{j-1}>0$ (where $D_{j}$ is the upper size limit), so that the total material in the sample, $v_{n}$, is sizecharacterized by the sum of the increments: 


$$
v_{n}=\sum_{D_{\min }}^{D_{\max }} \Delta v_{j}
$$

The size distribution is described by the cumulative vclume data $V\left(D_{j}\right)$ :

$$
v\left(D_{j}\right)=\sum_{D=0}^{D_{j}} \Delta v_{j}
$$

The particle size distribution normalized to the same volume is described by the measured data for (a) the incremental volume fractions, $\Delta v_{j} / v_{n}$ and (b) the cumulative volume fraction, $V\left(D_{j}\right) / v_{n}$. The empirical size distribution 18 typically presented graphically by a plot of the cumulative volume (or mass) fraction vs. particle diameter $D$, with a continuous line drawn through the data points to represent the continuous function, $V(D) / v_{n}$.

In the case of Coulter counter (CC) size analysis, the volume, $v$, of each particle is measured and the particle diameter, $D$ is calculated as that of a sphere of equal volume:

$$
D=\left(\frac{6}{\pi} v\right)^{1 / 3}
$$

The volume increments, $\Delta V_{j}$, of the particulate are calculated as the sum of particle volumes over the range of sizes defined by a constant ratio:

$$
\frac{D_{j}}{D_{j-1}}=2^{1 / 3}
$$

Such size increments are equal on a $\log$ scale of D. Thus, for CC data,

$$
\begin{aligned}
\Delta v_{j} & =\sum_{D_{j}-1}^{D_{j}} v_{j} \\
& =\frac{\pi}{6} N_{j}\left(\bar{D}_{j}\right)^{3}
\end{aligned}
$$

where $N_{j}$ is the total number of particles in this size range, $D_{j}-D_{j-l}$, and $\bar{D}_{j}$ is the mean size of this range, which is based on the mean particle volume, $\bar{v}_{j}$ : 


$$
\begin{aligned}
\bar{v}_{j} & =\frac{1}{2}\left(v_{j}+v_{j-1}\right) \\
& =\frac{\pi}{6}\left(\bar{D}_{j}\right)^{3}
\end{aligned}
$$

From Eqs. 3 through 6 , the mean size $\bar{D}_{j}$ or the volume increment, $\Delta v_{j}$, is a function of the upper-size limit, $D_{j}$, of the size range; thus,

$$
\overline{\mathrm{D}}_{\mathbf{j}}=0.909 \mathrm{D}_{\mathrm{j}}
$$

and correspondingly,

$$
\Delta V_{j}=0.393 \mathrm{~N}_{j}\left(D_{j}\right)^{3}
$$

In CC analysis, the incremental volume fractions, $\Delta \mathrm{v}_{j} / \mathrm{v}_{\mathrm{n}}$, are calculated for each of the CC size channels (indexed by the upper-limit size, $D_{j}$ ), from which the cumulative volume fractions, $V\left(D_{j}\right) / v_{n}$, are calculated and plotted. Typically, CC analysis is carried out on a sample consisting of only that part of a total fracture particulate which passes through a 90- $\mu \mathrm{m}$ mesh. The CC size analys is based on sample size, $v_{n}$, can be renormalized to the total volume, $v_{0}$, of the fracture particulate:

$$
\frac{v\left(D_{j}\right)}{v_{0}}=\frac{v\left(D_{j}\right)}{v_{n}} \frac{v_{n}}{v_{0}}
$$

Since CC analysis actually counts individual particles, the total mass of sample required is relatively small compared to other methods, e.g., sieving. When CC analysis is done in conjunction with sieving, the $-90 \frac{1}{\mu m}$ sample submitted for CC analysis can be weighed beforehand, and the value of $V_{n}$ in Eq. 9 can be obtained independently of the $v_{n}$ obtained internally by Eqs. 1 and 5 by the $C C$ instrument. The small sample requirement and the internal measurement of absolute values of sample volume $V_{n}$ are advantages of CC analysis. Certain other features of CC data are discussed later in connection with plots of combined CC and sieving data in lognormal graphical coordinates.

In sieve (SV) analysis, the entire volume (or mass) $v_{0}$ of the impacted specimen is used, and the absolute values of the volume increments by size, $\Delta v_{j}$, are determined by weighing. The size increments, $D_{j}-D_{j-1}$, are defined by a constant ratio,

$$
\frac{D_{j}}{D_{j-1}}=2
$$

which as in CC analysis, are equal linear increments on a scale of $\log D$. The mean size, $\bar{D}_{j}$, of the volume increment, $\Delta v_{j}$ is given by 


$$
\begin{aligned}
\bar{D}_{j} & =\frac{1}{2}\left(\mathrm{D}_{j}+\mathrm{D}_{j-1}\right) \\
& =0.75 \mathrm{D}_{j}
\end{aligned}
$$

In our measurements, we have combined $\mathrm{CC}$ data in the size range of $5 \mu \mathrm{m}$ to $100 \mu \mathrm{m}$ with sieving (SV) data for larger size particles, all nornalized to specimen volume, $V_{O}$. The combined data are used to determine the best values of the lognormal parameters, the geometric mean, $b_{g}$, and the geometric standard deviation, $\sigma_{g}$, as described in detail previously. The general method involved equating the cumulative normal probability function $P(u)$ with the empirical data provided by the cumulative volume fractions $V(\nu) / V_{0}$ described above. That is,

$$
P\left(u_{j}\right)=V\left(D_{j}\right) / v_{0}
$$

where the standardized normal variate $u$ is related to $D_{j}$ by the lognormal parameters, $\mathrm{D}_{\mathrm{g}}$ and $\sigma_{\mathrm{g}}$ :

$$
u_{j}=\frac{\log D_{j}}{\log \sigma_{g}}-\frac{\log D_{g}}{\log \sigma_{g}}
$$

Equation 13 shows a linear relation between $u$ and $\log D$, and this is the basis of the straight-line plots of particle size distributions on cumulative lognormal probability coordinates described in previous quarterly reports (see title page). The continuous lognormal probabllity function $P(u)$ can be used both graphically and analytically to interpolate and extrapolate the measured values of the cumulative volume fractions, $V\left(D_{j}\right) / v_{0}$.

The lognormal frequency function $p(u)$ is defined in terms of the cumulative probability function, $P(u)$, as

$$
\begin{aligned}
p(u) & =\frac{d P(u)}{d u} \\
& =\frac{1}{\sqrt{2 \pi}} e^{-u^{2} / 2}
\end{aligned}
$$

where $u$ has the same meaning as before. In terms of the normalized finite volume increments, $\Delta v_{j} / v_{o}$,

$$
\begin{aligned}
\frac{\Delta V_{j}}{V_{0}} & =\Delta P_{j} \\
& =P\left(u_{j}\right)-P\left(u_{j-1}\right) \\
& =p\left(\bar{u}_{j}\right) \Delta u_{j}
\end{aligned}
$$


Thus, the empirical values $\Delta v_{j} / v_{0}$ are related to the normal probability frequency function $p(u)$ by

$$
p\left(\bar{u}_{j}\right)=\frac{\Delta v_{j}}{v_{o}} \frac{\log \sigma_{g}}{\log \left(D_{j} / D_{j-1}\right)}
$$

where $\bar{u}_{j}$ is related to $D_{j}$ as defined for CC data in Eq. 7 and for SV data in Eq. 11. The empirical values, $\Delta v_{j} / v_{0}$, plot as straight lines on lognormal frequency coordinates.

The lognormal analysis of measured size distribution data from both CC and SV allows characterization of the fracture particulate in terms of the parameters, $D_{g}$ and $\sigma_{g}$. This has been demonstrated for numerous test data. The lognormal size distribution is mathematically consistent with a binarycleavage mode of fracture in impacts. That is, if it is assumed that every particle is the result of $n$ successive stages of three-dimensional binary cleavage in the portion of the impacted volume where the particle was formed, then the diameter, $D_{j}$, of that particle is related to $n$ and to the lognormal parameters by

$$
\begin{aligned}
\frac{D_{j}}{D_{g}} & =\left(\frac{1}{2}\right)^{n-n_{g}} \\
& =\sigma_{g} u_{j}
\end{aligned}
$$

where $\mathrm{n}_{\mathrm{g}}$ is the number of stages to form a particle of size $\mathrm{D}_{\mathrm{g}}$ from the original whole body of diameter, $D_{0}: D_{g} / D_{o}=\left(\frac{1}{2}\right)^{n_{g}}$. The lognormal characterization of the size distribution of a brittle-fracture particulate does not rest on any theoretical assumptions; it rests on empirical measurements. These implications for particle surface areas and for stress distributions will be developed in subsequent sections of this report. In the present section, the evaluation is directed to empirical particle size measurements.

One potential problem in combining Coulter counter (CC) data and sieving (SV) data is the fact that these methods determine particle diameter (D) differently. In CC analysis, $D$ is the equivalent spherical diameter of the measured particle volume. In SV analysis, D is the square mesh opening through which particles (of whatever shape) can fall under gravity while being agitated by mechanical vibrations. It can be shown by simple geometrical models that needle-shaped particles will have a smaller D in SV analysis than in CC analysis. In the case of plate-shaped particles, the reverse is true. This state of affairs is shown graphically in Fig. 18, for plots of cumulative volume fractions vs. particle size, which are linear on lognormal graphical coordinates. Actual measurements typically do not show major displacement of the lines, as illustrated for the calculated plate-shaped and needleshaped size distributions. Actual fracture particulate measurements appear to approximate a common straight line, as shown for a mixture of shapes, 

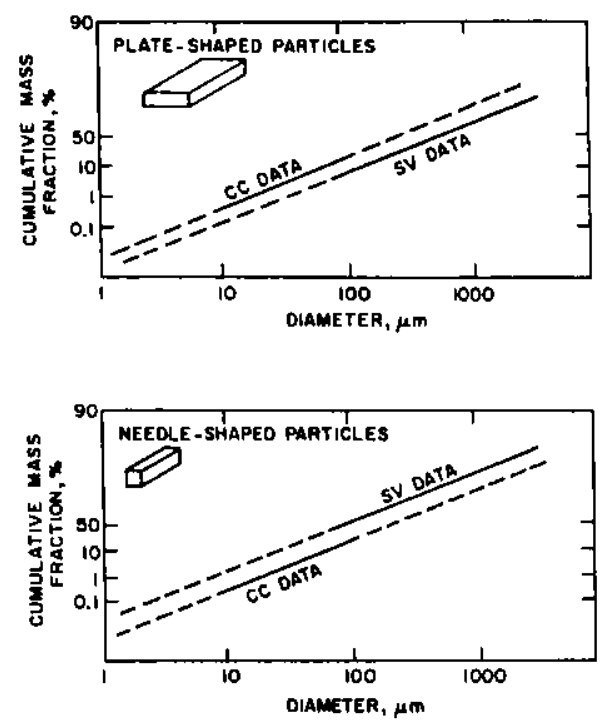

Fig. 18 .

Illustration of Shape Effect on Particle Size Distribution when Measured by Coulter Counter (CC) and Sieves (SV)

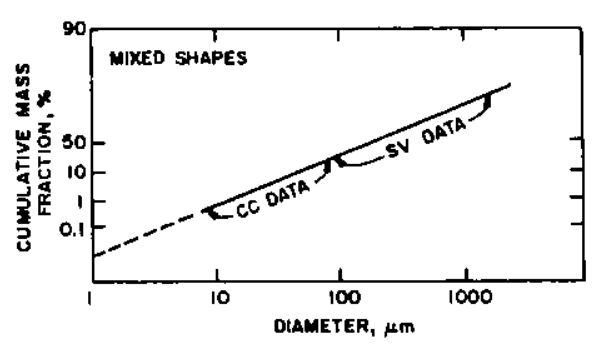

probably because shape variations tend to average out. Current presentation of empirical size distributions from combined CC and SV analysis have not been adjusted in any way; the actual data points (as in Fig. 16) indicate the extent to which this averaging effect takes place.

However, in the case of particles larger than about $2 \mathrm{~mm}$, there is a fundamental departure from linearity for impact tests. This effect, associated with the surface area of the fracture particulate, is discussed in the following section, but typical empirical results are illustrated in Fig. 19 . A typical size distribution is shown for (1) the cumulative mass function and (2) the cumulative surface area function of the lognormal fracture particulate, the latter of which consists of all particles smaller than size D98s and which includes more than $98 \%$ of the total surface area.

Also in Fig. 19, minor departures from linearity are seen at the extreme ends of the CC data, i.e., the downward concavities $D<10 \mu \mathrm{m}$ and at D $>70 \mu \mathrm{m}$. The former effect is due to the insensitivity of the Cc device to the measurement of particle volumes in the size range below about $5 \mu \mathrm{m}$. Thus, the cumulative volume fraction for the CC sample is inaccurate because material less than $5 \mu \mathrm{m}$ in size (whi .4 amounts to less than $1 \%$ of the total CC sample) is not detected. The practical result is to distort the shape of particle-size distribution curves for particles smaller than about $8 \mu \mathrm{m}$. This error of less than $1 \%$ in $V_{n}$, as determined internally by the $C C$, is not practically significant. The correct $V_{n}$ is known from the weight of material in the Counter counter sample, as discussed above in connection with Eq. 9. 


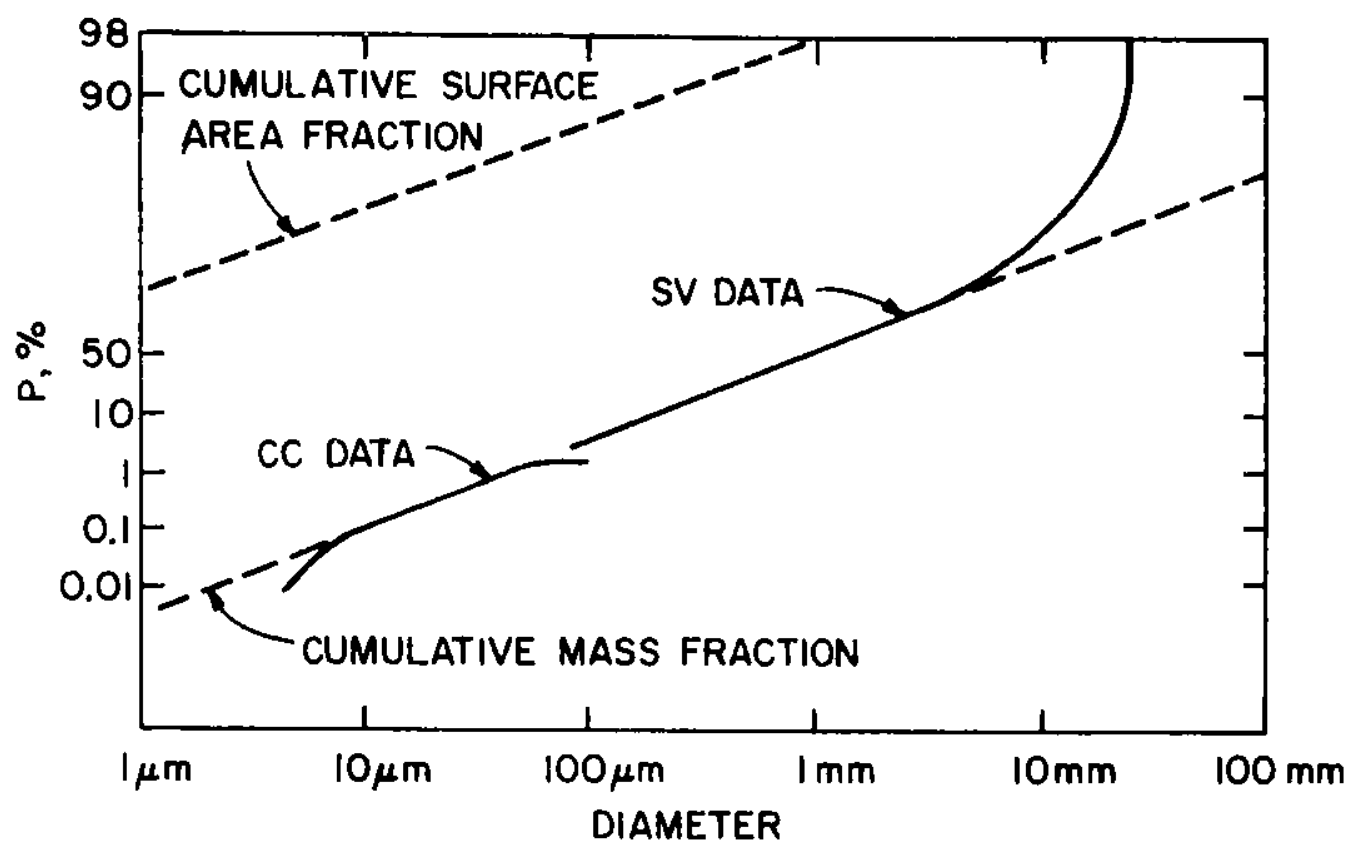

Fig. 19. Illustration of Lognormal Size Parameters in Relation to Measured Partic le Size Distributions. D98s $=1 \mathrm{~mm}$. $D_{\mathrm{L}}=5 \mathrm{~mm}$. Typically, $D_{g}$ is approximately equal to D98s. The slope of both straight lines $=1 / \log \sigma_{g}$.

The downward concavity of the CC data for $70 \mu \mathrm{m}<\mathrm{D}<100 \mu \mathrm{m}$ is due to an artifact of sieving. Typically, the CC sample is prepared by wet-sieving the fracture particulate to collect the $-90 \mu \mathrm{m}$ material. On the other hand, the sieving analysis itself is done dry, and additional material (not collected in wet sieving) falls through the $90-\mu \mathrm{m}$ sieve into the fines catcher. Although wet sieving provides efficient separation of small particles from large particles, it gives a different sieve calibration--that is, dry sieving allows larger particles to penetrate the $-90 \mu \mathrm{m}$ sieve than does wet sieving. Very properly, these nonlinear CC points should be ignored, so long as the first point of the SV data is an accurate cumulative volume fraction. Of course, when many points are used, the error of one point does not have a significant effect on the determination of $\mathrm{D}_{\mathrm{g}}$ and $\sigma_{\mathrm{g}}$. The above discussion is a reminder that accurate results depend on good calibration of all measurement devices used in particle size analysis.

3. Correlation of Lognormal Particle Size Parameters with Input Energy Density

\section{a. Energy Distribution in Impact Compression}

Brittle fracture is not a thermodynamically reversible process, nor can it be described in terms of chemical equilibrium states nor the continuous differential equations of physical mechanics. Nevertheless, the 
principle of conservation of energy applies to the transformations of the input kinetic energy and provides the basis for an overall description of the fracture process. This is described below, both for the general case of impact and for the use of testing machines.

In general, the stresses that generate fracture result from the force, $F$, applied to the brittle body by the deceleration of a mass, $M$, as it moves with decreasing velocity, $v$, as a function of $t$ ime, $t$ :

$$
\begin{aligned}
F & =M \frac{d v}{d t} \\
& =A_{f} \sigma
\end{aligned}
$$

where $\sigma$ is the stress at the load-bearing surface area, $A_{f}$, normal to the force axis. During the initial compression stage of impact, $F$ and $\sigma$ increase monotonically with time, because the elastic compression of fers continually increasing resistance to further movement. Maximum stress occurs when velocity $\mathrm{v}=0$.

In practice, typical brittle materials can be treated as homogeneous and isotropic in their elastic behavior. Hooke's law describes the linear relation of elastic stress, $\sigma_{x}$, and strain, $\varepsilon_{x}$, as a directional point property in a continuous medium.

$$
\sigma_{\mathrm{x}}=\mathrm{E} \varepsilon_{\mathrm{x}}
$$

where $E$ is Young's modulus, a constant for a given material. In a volume element, dxdydz, subject to normal stress (in the $x$-direction) only, the force on the element is the product of $\sigma$ and the area element dydz, and this force does work in producing a linear compression $\varepsilon_{\mathrm{x}} \mathrm{dx}$. For the volume element, this work $\mathrm{dW}$ is

$$
\mathrm{dW}=1 / 2\left(\sigma_{x^{\varepsilon}}^{\varepsilon}\right) \mathrm{d} x \mathrm{dyd} z
$$

The "l/2" in Eq. 20 is a consequence of the integration of the work (over $t$ ime) as strain goes from zero to $E$ within the volume element. From Eqs. 19 and 20 , we may write the strain energy density in the volume element, $d V=$ $\mathrm{d} x \mathrm{dyd} z$, as $\mathrm{dw} / \mathrm{dV}$ :

$$
\frac{d W}{d V}=\frac{\sigma_{x}^{2}}{2 E}
$$

Since we are concerned with application of a single unidirectional stress, the subscript $x$ is dropped in further expressions of stress, $\sigma$.

Saint-Venant's principle describes physical results of the local distribution of stress within a body, and the principle of energy conservation accordingly describes the total strain energy, $W$, in the body due to the applied force on the volume element in the volume integral of dW in $\mathrm{Eq} .21$ : 


$$
\mathrm{W}=\int \frac{\sigma^{2}}{2 \mathrm{E}} \mathrm{dV}
$$

If the force, $F$, is applied uniformly normal to the flat end of a cylinder of diameter, $D$, and length, $L$, the load-bearing surface is $A_{f}=\pi / 4 D^{2}$, the stress, $\sigma$, is uniform over the volume $V=A_{f} L$, and the work, $W$, is

$$
\mathrm{W}=\frac{\sigma^{2}}{2 \mathrm{E}} \mathrm{V}
$$

In Eq. 23, the stress, $\sigma$, is uniform, and so stress, $\sigma$, and energy density, $\mathrm{W} / \mathrm{V}$, are mutually defined. In $\mathrm{Eq} .22$, the overall energy density is also $\mathrm{W} / \mathrm{V}$, wh ich defines a volume mean stress, $\bar{\sigma}$, corresponding to:

$$
\frac{W}{V}=\frac{\sigma^{2}}{2 E}
$$

This has significance with respect to the impact-fracture process. If the fracture results correlate with the mean stress, then the overall energy density determines the fracture results, independent of the actual stress distribution. This appears to be so, at least under some impact conditions, and it is a fortunate result because dynamic stress distributions are in practice indeterminate.

The above consideration of strain energy density shows that for any body shape or size, the overall stress state is defined by the overall energy density, $W / V$, where $W$ is equal to the input energy. For impact compression alone, the loss of kinetic energy is equal to the overall strain energy, and the mean stress is the same for all configurations, as defined in terms of overall energy density, $W / V$. For example, diametral compression of a cylinder (between flat plates) gives the same overall energy density as for axial compression, even though the peak stress and the stress distribution are different.

If the chief practical interest were to characterize the structural strength of a brittle material in impacts, then the proper experimental test would be to determine the threshold stress for fracture under various controlled dynamic load conditions. However, if the practical interest is to characterize the way impacts of various severities result in the formation of a particulate (measured by surface area and respirable fraction), then the stress per se is not importatut, even though stress is the driving force for brittle fracture. In this impact-fracture process, stress varies greatly with both time and location in the compressed body, and the first crack propagation destroys the continuous stress field, so that stresses during crack propagation are actually indeterminate. In practice, even the threshold stress cannot be made uniform in the axial impact of a specimen, because of small directional misalignments and local surface-contact perturbations of the stress field in the compressed material.

As described above, impact severity is measured overall for the specimen by stress energy density, which is a direct correlate of the mean stress in the material. This mean stress is anticipated to correlate 
with the mean size, $D_{g}$, and mean shape factor, $a$, as characterized stat istically. Only experimental tests will establish adequate experimental procedures for characterizing impact results.

\section{Stress Distributions Calculated from Particle-Size Distributions}

The preceding sections show how impact energy is converted to strain energy density (which is equivalent to stress) and how the total fracture surface is related to the lognormally distributed fracture particulate. If impact conditions are such that there are no significant pathways for energy dissipation other than brittle fracture, then the impact kinetic energy will be "used up" by being converted into strain energy, which is then dissipated into heat by collisions of the particles ejected by the impact. The extent of fracture measured by the total fracture surface area, $S_{f}$, may then be correlated with impact energy density, $w_{i} / v_{0}$ :

$$
\varepsilon \frac{W_{i}}{V_{o}}=\gamma_{f}\left(\frac{S_{f}}{V_{o}}\right)
$$

where $\varepsilon$ is an efficiency factor (allowing for some dissipation of input energy to the surroundings) and $Y_{f}$ is an impact resistance property (reported to be $77 \mathrm{~J} / \mathrm{m}^{2}$ for Pyrex glass and for mineral quartz) [ZELENY].

The total surface area, $S_{n}$, of the lognormally distributed fracture particulate is equal to $S_{f}$, the total fracture-cleavage surface area. Mathematically, $S_{n}$ is related to $v_{n}$ by the lognormal parameters, $D_{g}$ and $\sigma_{g}$ :

$$
\begin{aligned}
\frac{\varepsilon}{\gamma_{f}}\left(\frac{W_{i}}{V_{n}}\right) & =\frac{s_{n}}{V_{n}} \\
& =\frac{{ }^{\alpha \sigma_{g}} 0.5 \ln \sigma_{g}}{D_{g}}
\end{aligned}
$$

which gives the overall relation for impact energy and particle characteristics, including surface area. If we consider a volume increment of the fully compressed material about to fracture in the particulate mode, the local energy density, $\Delta W / \Delta V$, corresponds to local stress by Eq. 23:

$$
\frac{\Delta W}{\Delta V}=\frac{a^{2}}{2 E}
$$

We may also apply the overall relation between particulate surface area and energy density given in Eq. 26 to apply to this volume increment:

$$
\left(\frac{\varepsilon}{\gamma_{f}}\right) \frac{\Delta W}{\Delta V}=\frac{\Delta S}{\Delta V}
$$


The surface and volume increments, $\Delta S$ and $\Delta V$, are related to the lognormal distribution of particle diameter, D. In the notation introduced previously,

$$
\begin{aligned}
\frac{\Delta V}{v_{n}} & =\frac{V\left(D_{j}\right)}{v_{n}}-\frac{V\left(D_{j-1}\right)}{v_{n}} \\
& =P\left(u_{v, j}\right)-P\left(u_{v, j-1}\right) \\
\frac{\Delta S}{S_{n}}=\frac{S\left(D_{j}\right)}{S_{n}}-\frac{S\left(D_{j-1}\right)}{S_{n}} & P\left(u_{v, j}+\ln \sigma_{g}\right)-P\left(u_{v, j-1}+\ln \sigma_{g}\right)
\end{aligned}
$$

For factor-of-two size increments,

$$
u_{v, j-1}=u_{v, j}-\frac{\ln 2}{\ln \sigma_{g}}
$$

and $\Delta S / \Delta V$ is defined for each value of $u_{v}$ (a function of $D$ ).

Combining Eqs. 27 through 31,

$$
\begin{aligned}
\frac{\sigma^{2}}{2 E} & =\left(\frac{\varepsilon}{\gamma_{f}}\right)\left(\frac{\Delta W}{\Delta V}\right) \\
& =\frac{\Delta S}{\Delta V} \\
& =\frac{P\left(u_{v}+\ln \sigma_{g}\right)-P\left(u_{v}+\ln \sigma_{g}-\ln 2\right)}{P\left(u_{v}\right)-P\left(u_{v}-\ln 2\right)}
\end{aligned}
$$

so that $\sigma=f\left(u_{v}\right)=f(D)$. Thus, for given values of the parameters in these equations, $\sigma$ is defined in terms of particle size. That is, there is a frac$t$ ion of the specimen volume, given by $P\left(u_{v}\right)=v(D) / V_{n}$, which has a particle size less than D. Correspondingly, that fraction of the volume is stressed at a level of at least $\sigma=f(D)$, as given by Eq. 32 .

A plot of such stress destruction calculations was made for a test of Savannah River Laboratory 131 waste $\mathrm{glass,} \mathrm{for} \mathrm{which} \mathrm{D}_{\mathrm{g}}=2.6 \mathrm{~mm}, \sigma_{\mathrm{g}}=$ $6.4, \alpha=30, \gamma_{f}=77 \mathrm{~J} / \mathrm{m}^{2}, \varepsilon=1.0$, and $E=7 \times 10^{10} \mathrm{~Pa}$. The results are plotted in Fig. 20.

It is as sumed that each particle is formed by multiple stages of three-dimensional binary cleavage of the original body of diameter $D_{\sigma}$,

$$
\frac{\mathrm{D}}{\mathrm{D}_{0}}=\left(\frac{1}{2}\right)^{\mathrm{n}}
$$




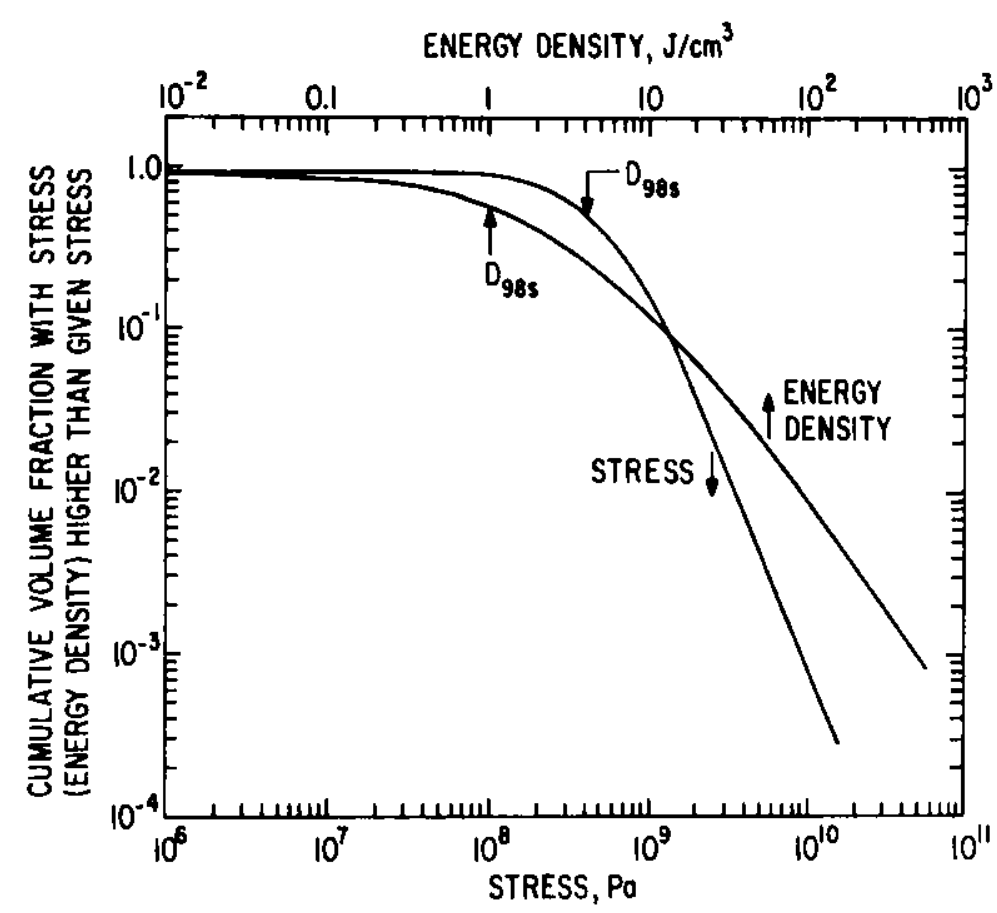

Fig. 20. Volume Distribution of Stress and Energy Density for Sample of SRL 131 Waste Glass

where $\mathrm{n}$ is the number of stages of binary cleavage. A similar relation of the lognormal parameters is

$$
\frac{D}{D_{g}}=\sigma_{g}^{u} v
$$

Equations 33 and 34 define the relation between $u$ and $u_{v}$ for a given $b_{1}$. For given values of $\mathrm{D}_{\mathrm{g}}$ and $\sigma_{\mathrm{g}}$, the values of the distribution of $\mathrm{n}$ as a function of volume are plotted in Fig. 21. The plot indicates that $50 \%$ of the volume has had at least two stages of binary cleavage, but only $0.2 \%$ of the specimen volume has had more than ten stages of binary cleavage. The cumulative surface area fraction as a fraction of the cumulative volume fraction (starting from the smallest size particles) is also plotted in Fig. 21.

The significance of these calculations depends on whether the results are consistent with all of the available information and whether correlations for the values of the parameters have predictive values. One possibility is that the range of particle sizes may be related to the range of stress levels in the material. This can only be established by systematic experimental tests.

One other calculation of possible significance can be made if particle diameter, D98s, as shown in Fig. 20, is taken as defining the upperlimit size of the fracture particulate proper. The stress corresponding 


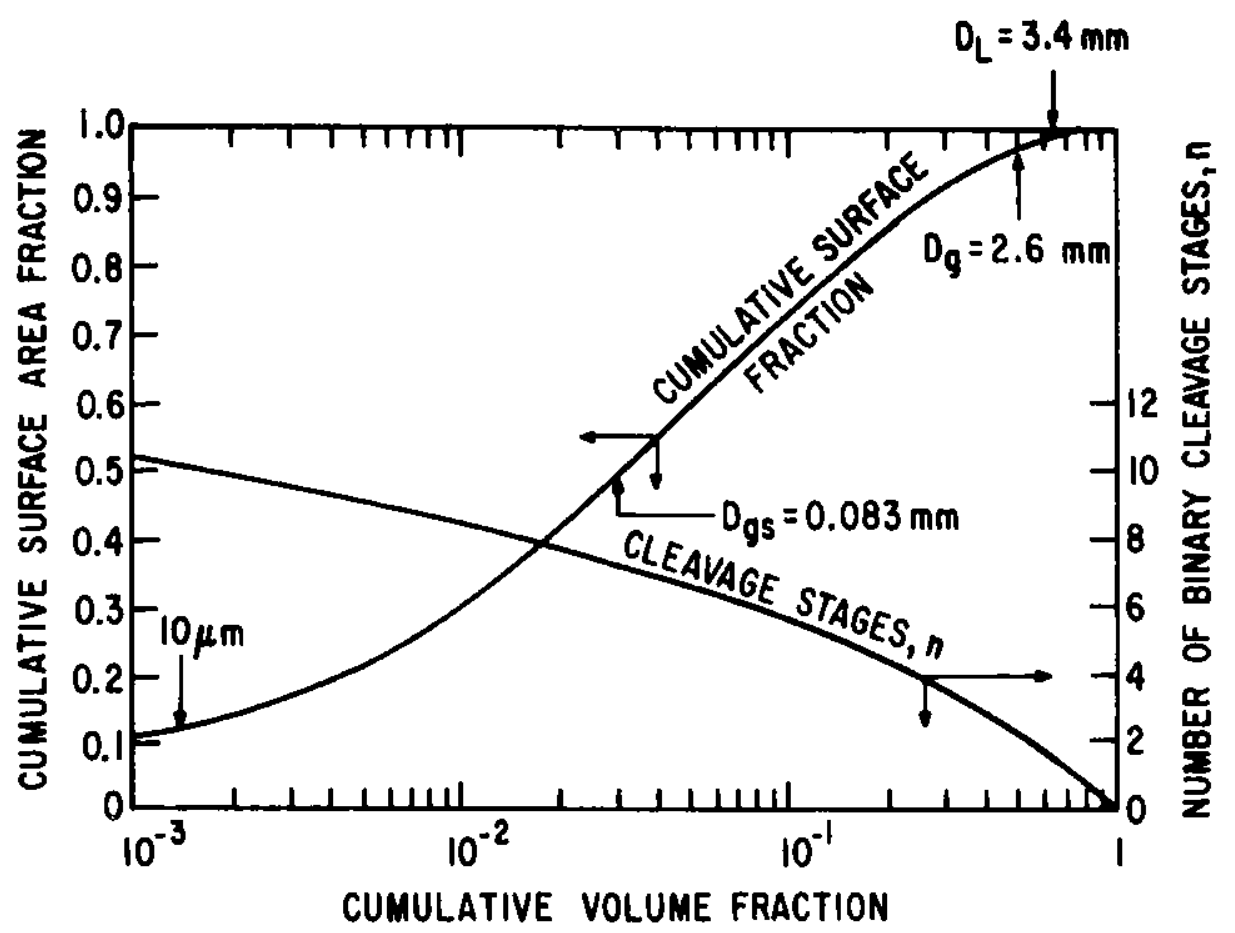

Fig. 21. Volume Distribution of Surface Area and Number of Binary Cleavage Stages

to this value of $D$ can be considered the threshold stress for particulate fracture. It is marked in Fig. 18, indicating a value of $4 \times 10^{8} \mathrm{~Pa}$ or $58,000 \mathrm{psi}$. This is a reasonable value for a threshold fracture stress in impacts.

\section{Energy Partitioning in Impact Test Devices}

The initial compression stage of impacts of brittle specimens has been analyzed above without consideration of the deformation of the surroundings. In this section, the principal ways in which the surroundings interact with the specimen during compression and brittle fracture are identified. Since the deceleration force causing stress and fracture in the specimen is also borne by the components of the surroundings in contact with the specimen, these components are subject to elastic compliance or plastic deformation, which reduces the severity of impact on the specimen. In impact tests, the principal components of the test device are chosen for their resistance to elastic and inelastic deformation. It is assumed here that these components have the properties of typical hardened steel and consist of an anvil upon which the specimen rests and a hammer which strikes the specimen. The general configuration is shown in Fig. 22.

Dynamic force loading in test devices can be characterized according to the degree of compliance of the steel hammer and anvil. As the force is increased, compressive stress increases in both the specimen and the steel. Typically, the steel deforms elastically but does not deform plastically. When stress in the specimen exceeds the elastic limit, the specimen fractures and the pieces are ejected in a direction normal to the force axis, which is 
(a) NO FORCE

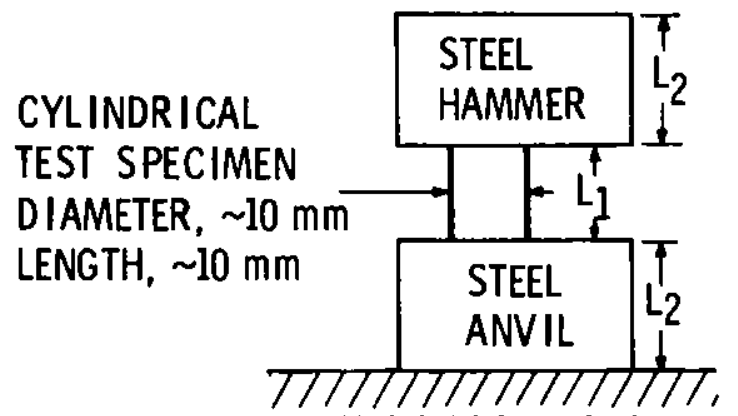

UNYIELDING SURFACE (b) IMPACT FORCE

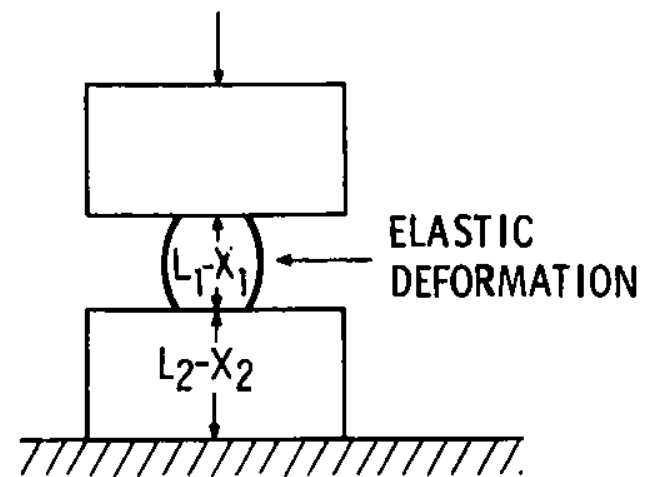

NOMINAL DIMENSIONS

$L_{1}$ = length (along force axis) of specimen

$L_{1}=$ length (along force axis) of each steel

LINEAR DEFORMATION UNDER COMPRESSIVE FORCE $F$

$X_{1}=$ approximate value for specimen

$X_{2}=$ approximate value for steel

Fig. 22. Model of Impact Test Device

the only "open" space to which they can move. This "fracture dislocation" relieves the elastic stress in the specimen and also relieves the force holding the hammer and anvil in compression at the contact surfaces between them and the specimen. The hammer and anvil immediately expand to achieve a new force balance, and the amount of such expansion depends on the compliance of the steel. In a "perfectly hard" loading, with no compliance at all in the steel components, the resistance force presented to the hammer and anvil drops to zero and no further compressive energy is "fed into" the specimen after the initial fracture event. In real tests, there is some compliance and there may be some residual force presented to the hammer and anvil, depending on the downward motion of the hammer and on the speed with which the specimen disintegrates. If there is no mechanical stop between the hammer ind the anvil, the hammer will finally come to rest atop whatever brittle fragments have failed to escape the hammer's path. Clearly, impact fracture in such a case consists of several stages of impact. In drop-weight impact tests of this type, the total extent of fracture is that which results from the complete dissipation of kinetic energy, however the fracture process occurs. Motion pictures, hosever, indicate that the primary fracture "explosion" is the dominant event and that successive stages probably produce relatively little fracture. 
If a mechanical stop 1 imits the travel of the hammer, the secondary crushing stage is avoided. However, depending on the compliance of the steel and the fracture behavior of the brittle material, there may be some addi$t$ ional feed-in of energy to the specimen after fracture has commenced. The elastic compression stage is analyzed below, and an estimate is made of the minimum compliance of the steel relative to that of the specimen. Also estimated is the partitioning of compression energy between the specimen and the steel at the point of maximum developed force, when fracture is just beginning. Although the complete fracture process takes place in a fraction of a millisecond in typical test specimens, the force can be measured as a function of $t$ ime with a high-speed piezoelectric force transducer with video readout. Force measurement with in about $1-\mu \mathrm{s}$ time resolution can be achieved. The fluctuations of force during impact give information on the stagwise progress of brittle fracture in tests.

In Fig. 22(a), the general configuration is shown under the zero force conditions. In Fig. 22(b), the elastic deformation is shown under maximum force, assuming that fracture has not developed sufficiently to distort the stress fields. (The deformation, as shown, is exaggerated for illustrat ive purposes.)

Since compressive forces propagate at speeds up to the acoustic velocity (which for a typical glass is about $5000 \mathrm{~m} / \mathrm{s}$ ), a force balance is approximately maintained even under the rapid and brief decelerations involved in impact tests. This balance of force, $F$, in the specimen and the steel can be expressed in terms of the dimensions identified in Fig. 22 by the following equations, where $E_{1}$ is the elastic modulus of the specimen and $E_{2}$ that of steel:

$$
\begin{aligned}
F & =\frac{A_{1} E_{1} X_{1}}{L_{1}} \\
& =\frac{A_{2} E_{2} X_{2}}{L_{2}}
\end{aligned}
$$

The compressive work $W_{1}$ in the specimen and $W_{2}$ in the steel are given by

$$
W_{1}=\frac{A_{1} E_{1} X_{1}^{2}}{2 L_{1}} ; W_{2}=\frac{A_{2} E_{2} X_{2}{ }^{2}}{2 L_{2}}
$$

The ratio of $\mathrm{W}_{2} / \mathrm{W}_{1}$ is thus

$$
\begin{aligned}
\frac{W_{2}}{W_{1}} & =\frac{X_{2}}{X_{1}} \\
& =\frac{A_{1} E_{1} L_{2}}{A_{2} E_{2} L_{1}}
\end{aligned}
$$


Typical specimen dimensions are of the order of $1-\mathrm{cm}$ diameter and length, and specimens have an elastic modulus smaller than that of steel. The estimate of the minimum ratio of $W_{2} / W_{1}$ is the product of the estimated minimum values for the component ratios as follows:

$$
\frac{A_{1}}{A_{2}}=0.1 ; \frac{L_{2}}{L_{1}}=4 ; \frac{E_{1}}{F_{2}}=0.25
$$

Therefore,

$$
\frac{\mathrm{w}_{2}}{\mathrm{w}_{1}}>0.1
$$

and the fraction of the total work of elastic compression which is stored in the steel is

$$
\frac{2 W_{2}}{2 W_{2}+W_{1}}>18 \%
$$

This shows a relatively small fraction but not a negligible amount of total energy is stored. A more typical set of dimensions would give $\mathrm{W}_{2} / \mathrm{W}_{1}=0.6$, or possibly a higher value. The partitioning of energy limits the stress in the specimen, depending on the impact-energy impact. In addition, the energy stored in the steel can "feed in" to the specimen during fracture, and thus can contribute to the fracture results.

In the technical literature relevant to force-instrument testing devices, an analysis is given which relates (1) the measured force to (2) the energy input to the test specimen. This analysis does not consider the energy partition outlined above, but is of interest in describing the test dynamics, particularly when a force transducer is used.

In general, the compression force, $F$, is determined by the moving mass, $M$ (typically, the hammer), and its velocity, $v$, as a function of $t$ ime, $t$, that is,

$$
F=M \frac{d v}{d t}
$$

The time integral of force, $I_{F}$, is equal to the $108 s$ of momentum (Mv) from the original velocity, $v_{0}$, to $v(t)$ :

$$
\begin{aligned}
I_{F} & =\int_{0}^{t} F d t \\
& =M v_{0}-M v
\end{aligned}
$$


The original kinetic energy is $1 / 2 \mathrm{Mvo}_{\mathrm{o}}^{2}$, and the loss of kinetic energy, $\mathrm{W}$, corresponding to the loss of momentum is

$$
W=1 / 2 M v_{0}^{2}-1 / 2 M v^{2}
$$

where $\mathrm{v}$ can be found from Eq. 42:

$$
v=v_{0}-\frac{I_{F}}{M}
$$

Substitut ing v from Eq. 44 in Eq. 43 ,

$$
\begin{aligned}
W & =v_{0} I_{F}-\frac{I_{F}^{2}}{2 M} \\
& =v_{0} I_{F}\left(1-\frac{v_{0} I_{F}}{2 M v_{0}^{2}}\right)
\end{aligned}
$$

The mean velocity, $\bar{v}$, can be defined in terms of Eq. 44:

$$
\begin{aligned}
\bar{v} & =\frac{v_{0}}{2}+\frac{v}{2} \\
& =v_{0}-\frac{I_{F}}{2 M} \\
& =v_{0}\left(1-\frac{v_{0} I_{F}}{2 M v_{0}^{2}}\right)
\end{aligned}
$$

$\overline{\mathrm{v}}$ of Eq. 46 can be substituted in Eq. 45 :

$$
\begin{aligned}
\Delta W & =\bar{v} I_{f} \\
& =\bar{v} \int_{0}^{t} F d t
\end{aligned}
$$

If $\mathrm{v}$ does not change much in this time interval, $\bar{v} \doteq \mathrm{v}_{0}$, and from Eq. 47 ,

$$
\Delta W=v_{0} \int_{0}^{t} F d t
$$


where the loss of kinetic energy is associated with the gain of elastic strain energy in the compressed specimen. Although Eq. 48 is sometimes said to define the energy input into the test specimen in terms of the readings of a force transducer, it is evident from the above mathematical basis that such a claim makes two assumpticns: (1) the mean velocity, $\bar{v}$, in the time period is known and (2) the steel components of the test device are perfectly hard and do not share any of the elastic strain energy. In general, these conditions do not hold, although for some impact conditions they may be close enough approximations that test results so interpreted are not seriously in error with respect to the energy input to the specimen.

The direct measure of force does indicate the level of mean stress in the specimen at the time when the decrease of force indicates that fracture is extensive. The calculation of energy density from the mean stress (and vice versa) is discussed in Section III.C.4 above. Impact tests may be arranged so that stresses in the test specimens are approximately uniform. As mentioned above, it is hard to verify such uniformity. Probably, only consistent test results will show the consequences of nonuniform stress in impact. Possibly, the range of particle sizes in axial impacts of cylindrical specimens will indicate the nonuniformity of the stresses causing fracture.

In accidents, 1mpact stresses are not uniform. A methodology for characterizing the severity of 1mpact in terms of ( 1 ) overall impact energy density, (2) body size, and (3) the configuration of impact is being developed. 
IV. FLUIDS IN ROCK

(M. G. Seitz, D. L. Bowers, *

and C. L. Herzenberg ${ }^{\dagger}$ )

Work is being conducted to test the performance of proposed nuclearwaste repositories using laboratory-analog experiments and to improve nuclear logging methods for oil-depleted oil reservoirs. Final plans were made for the seminar, Hazardous-Waste Management, to be held at Argonne July 15 and 16,1981 , for university faculty.

\section{A. Repository-Analog Experiments}

Work conducted in preparation for the repository-analog experiments to be conducted for the U.S. Nuclear Regulatory Commission included (1) the development of a data acquisition system for monitoring the experiments and (2) preparation of aqueous fluid that is representative of groundwater in the Pasco Basin, Washingt on state, for the experiments.

The data acquisition system is based on (1) a Digital Equipment Central Processing Unit with disc storage and (2) Crate analog-to-digital conversion systems. The acquisition system will record temperatures and pressures during the experiments. The system is currently located in the Chemical Engineering Division building for testing and preliminary use.

\section{B. Water for the Analog Experiments}

A solution was prepared to simulate groundwater found in the Grande Ronde formation of the Columbia Basin [GEPHART]. The salts 1 isted in Table 14 were dissolved in $20 \mathrm{~L}$ of pure (about $18 \mathrm{~m} / \mathrm{cm}$ ) water. Because few silica compounds are readily dissolved, sodium $s$ ilicate was chosen as the source of silicate. Us ing this compound causes the total sodium concentration to be higher than that 1 isted for the reference groundwater $(330 \mathrm{mg} / \mathrm{L}$ vs. $250 \mathrm{mg} / \mathrm{L})$. Analytical reagent grade chemicals were used to prepare the solution (Table 14).

The concentrations of the individual anions and cations were calculated and are reported in Table 15 along with the concentrations in the reference groundwater.

The solution was sparged with nitrogen for approximately five hours. The pHs at both the top and bottom of the solution were measured: $\mathrm{pH}(t o p)=$ 11.06 and $\mathrm{pH}$ (bottom) $=11.03$. The $\mathrm{pH}$ is higher than the reference value ( $\mathrm{pH}$ of 10.1 ), but this was expected because of the extensive sparging with nitrogen and because of the addition of sodium silicate.

\section{We11 Logging}

Preparations were made during the final month of the quarter to conduct core-flood experiments. Laboratory space was obtained for work with radiochemicals, and the pumps, rock-core holder, and other apparatus for the

\footnotetext{
* Member of the Analytical Group of Chemical Engineering Division.

'Member of the Applied Physics Division.
} 
Table 14. Compounds Used to Formulate Solution Similar to the Groundwater in the Grande Ronde Formation

\begin{tabular}{lc}
\hline Compound & $\begin{array}{c}\text { Quantity Added } \\
\text { to } 20 \mathrm{~L} \\
\text { of Water }\end{array}$ \\
\hline Sodium chloride, $\mathrm{NaCl}$ & $4.8866 \mathrm{~g}$ \\
Sodium Sulfate, $\mathrm{Na}_{2} \mathrm{SO}_{4} \cdot 10 \mathrm{H}_{2} \mathrm{O}$ & $7.2493 \mathrm{~g}$ \\
Sodium borate, $\mathrm{Na}_{2} \mathrm{~B}_{4} \mathrm{O}_{7} \cdot 10 \mathrm{H}_{2} \mathrm{O}$ & $0.2458 \mathrm{~g}$ \\
Sodium fluoride, $\mathrm{NaF}$ & $1.6361 \mathrm{~g}$ \\
Sodium carbonate, $\mathrm{Na}_{2} \mathrm{CO}_{3}$ & $1.2013 \mathrm{~g}$ \\
Sodium bicarbonate, $\mathrm{NaHCO}_{3}$ & $1.1844 \mathrm{~g}$ \\
Sodium silicate, $\mathrm{Na}_{2} \mathrm{SiO}_{3} \cdot 9 \mathrm{H}_{2} \mathrm{O}$ & $11.4622 \mathrm{~g}$ \\
Potassium sulfate, $\mathrm{K}_{2} \mathrm{SO}_{4}$ & $0.0853 \mathrm{~g}$ \\
Magnesium sulfate, $\mathrm{MgSO}_{4} \cdot 7 \mathrm{H}_{2} \mathrm{O}$ & $3.58 \mathrm{mg}$ \\
Calcium sulfate, $\mathrm{CaSO}_{4} \cdot 2 \mathrm{H}_{2} \mathrm{O}$ & $0.112 \mathrm{~g}$ \\
\hline
\end{tabular}

Table 15. Concentrations of Constituents in Aqueous Solution and in Groundwater of the Grande Ronde Formation

\begin{tabular}{|c|c|c|}
\hline $\begin{array}{l}\text { Element or } \\
\text { Compound }\end{array}$ & $\begin{array}{l}\text { Aqueous } \\
\text { Solution } \\
\text { Calculated } \\
\text { Values, } \\
\text { mg/L }\end{array}$ & $\begin{array}{l}\text { Reference Values for } \\
\text { Groundwater of the } \\
\text { Grande Ronde } \\
\text { Structure, a } \\
\text { mg/L }\end{array}$ \\
\hline $\mathrm{Na}$ & 330 & 250 \\
\hline $\mathrm{Cl}^{-}$ & 148 & 148 \\
\hline $\mathrm{So}_{4}^{2-}$ & 114 & 108 \\
\hline B & 1.39 & 1.39 \\
\hline $\mathbf{F}^{-}$ & 37 & 37 \\
\hline $\mathrm{CO}_{3}^{2-}$ & 34 & 34 \\
\hline $\mathrm{HCO}_{3}^{-}$ & 43 & 43 \\
\hline $\mathrm{SiO}_{2}$ & 121 & 121 \\
\hline $\mathbf{K}$ & 1.9 & 1.9 \\
\hline $\mathrm{Mg}$ & 0.04 & 0.04 \\
\hline $\mathrm{Ca}$ & 1.3 & 1.3 \\
\hline
\end{tabular}

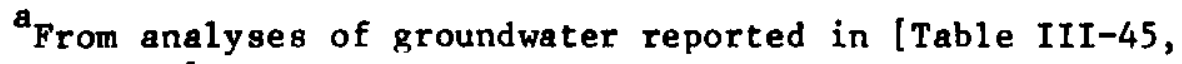
GEPHART ] . 
experiments were collected. The experiments are designed to test the proposed method of injecting gamma-active solution into a reservoir to measure residual oil (STEINDLER-1981C). The objective of the experimental program is to determine if the gamma signal from rock injected with radioactive fluid depends on the oil content of the rock.

Ten samples of Berea sandstone obtained for the work appear to have a uniform appearance and uniform density. The ten samples have an average density of $2.098 \mathrm{~g} / \mathrm{cm}^{3}$ with a pooled variance of $0.005 \mathrm{~g} / \mathrm{cm}^{3}$. In comparison, an error in density of $0.039 \mathrm{~g} / \mathrm{cm}^{3}$ is estimated from the error expected in we ight and dimension measurements. Thus, the densities of the ten samples appear to be the same. Porosity (based on a density of $2.655 \mathrm{~g} / \mathrm{cm}^{3}$ for quartz) was estimated to be $21 \%$.

The injection medium chosen for the core flood tests is an aqueous solution with a composition similar to that of a brine reservoir fluid. A long-chain hydrocarbon surfactant, Tricon $X-100,{ }^{*}$ will be added to give the aqueous solution favorable surface properties to effectively displace petroleum. A surfactant concentration of $1 \times 10^{-4}$ mol was chosen. The choice was made on the basis of the low (22 dynes/cm) oil-water interfacial surface tension displayed by the surfactant at that concentration. The surfactant is nonionic and therefore should not adsorb to a great degree onto the quartz surfaces (previous rock mechanics experiments by Dunning and others seem to confirm this). Also, the surfactant should not complex readily with the $22 \mathrm{Na}$ radiotracer.

\section{Experimental Series}

Four cores will be used in the experimental series. In the planned tests, two of the cores will be saturated with the crude oil and inserted into the jacketed vessel assembly. The cores will be "produced" by injecting the aqueous solution into the cores. This will establish a condition comparable to the situation existing in a watered-out reservoir. The other two cores, containing no oil, will be saturated with the aqueous solution. Then all four cores will be injected with sodium-22 bearing aqueous fluids, and "logged" using a scintillation crystal and electronic counting equipment. The gamma signals of oil-saturated cores will be compared with signals detected on cores that do not contain oil. The difference in signals will be analyzed to determine the net effect of the oil present in two of the cores. The two cores containing oil are expected to have diffrent residual-oilsaturation values because of the use of surfactant in one of the cores.

* The surfactant is made by Rohm and Haas. 


\section{TRACE-ELEMENT TRANSPORT IN LITHIC MATERIAL BY FLUID FLOW \\ (M. G. Seitz and R. A. Couture)}

\section{A. Introduction}

In the preceding report in this series [STEINDLER-1981C], we reported on the composition of pore water eluted from a core sample of Precambrian granite from Northern Illinois drill hole UPH-3. It was found that the pore water is $\mathrm{NaCl}-\mathrm{CaCl}_{2}$ brine $\left(\mathrm{Cl}^{-}>0.24 \mathrm{M}\right)$, and that the water is extraordinarily high in fluoride (at least $3.3 \overline{\mathrm{mM}}$ ). However, the absolute concentra$t$ ion of salt in the brine was not determined-only the ionic ratios. It was suspected that the water may have been influenced by brine in overlying sediments (although no brine was reported in sediments in $h$ is hole), or that the brine may be representative of old, granitic groundwater, which derived its unique composition by long reaction with the granite.

In this report, we discuss the origin of the brine, and we present work on some experimental refinements. We consider methods for determining the absolute concentrations of ions in the pore water and the effective pore volume, and we present calculations on the rate of drying of the samples after sampling.

We have also begun some tests to estimate the rate of leakage around the rock core; the data will be given in the next report when the work is complete.

On another subject, evidence for trace-element migration from the Notch Peak Intrusion of Western Utah is summarized briefly. This work was carried out primarily by James Papike and coworkers; permeability measurements in our laboratory and field work by M. Seitz were discussed previously [STEINDLER$1981 \mathrm{~A}, 1981 \mathrm{~B}]$.

\section{B. Origin and Significance of the Brine}

The composition of the pore water from drill hole UPH-3 more closely resembles that of sedimentary brine than that of brine from granitic rocks from the Sudbury region of the Canadian Shield (Fig. 23; [FRAPE]). It is suggested that the water was derived from the overlying Cambrian sediments, which are very briny, especially in the deeper parts [MEENTS, BOND, ILLINOIS] . The fluoride in the water may be related to the presence of up to $2 \%$ fluorite $\left(\mathrm{CaF}_{2}\right)$ present in the granite as a late-stage mineral. The presence of fluoride has been confirmed with a fluoride-specific electrode: in eluate fraction 11 [STEINDLER-1981C], $0.35 \pm 0.04 \mathrm{mM} \mathrm{F}$ was found by ion chromatography, compared to $0.32 \pm 0.05 \mathrm{mM}$ by electrode (Florence Williams, analyst).

The high concentrations of $\mathrm{F}^{-}$in the presence of $\mathrm{Ca}^{2+}$ imply a complexing agent for $\mathrm{F}^{-}$. No trivalent elements were present in the solutions, and the concentration of $\mathrm{Mg}^{2+}$ is lower than that of $\mathrm{F}^{-}$. A survey of complex formation constants suggests that the most likely complexing agent is boron, with silica a possibility. The detection limit for boron was too high for measurement, and fluoride is known to coexist with calcium and boron in water from the Mt. Simon aquifer [ILLINOIS]. Incidentally, tourmaline (a borosilicate) is present in the granite. This fact may be irrelevant, but it shows that there is boron in the system. We tentatively consider the composition of the 


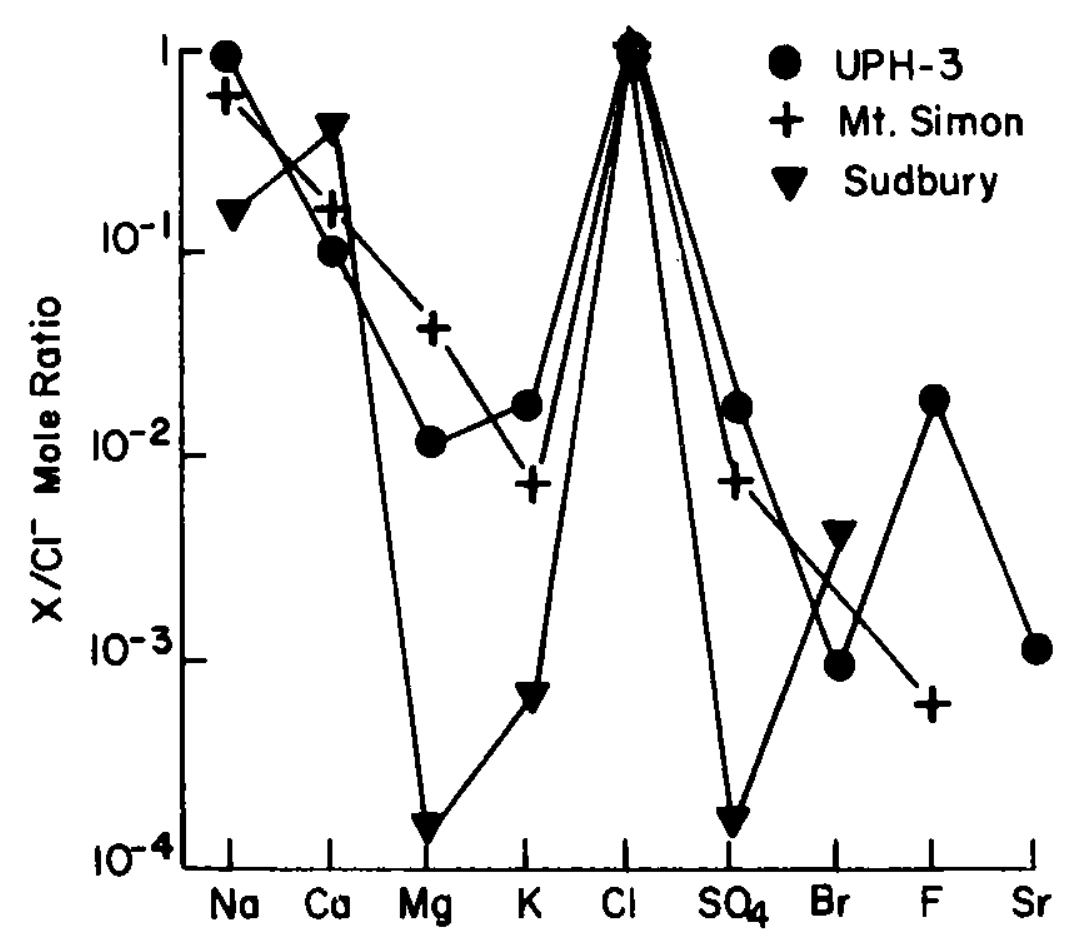

Fig. 23. Composition of Groundwater and Pore Water from Precambrian Granite, $1288 \mathrm{~m}$, Dril1 Hole UPH-3, Stevenson Co., I11.; from Mt. Simon Aquifer, $1225 \mathrm{~m}$, Du Page Co., I11. [ILLINOIS]; and from a Diabase Dike, $1219 \mathrm{~m}$, Sudbury Area, Ontario [FRITZ]. $\mathrm{Ca} / \mathrm{Cl}$ ratio in UPH-3 is much lower than in any saline water reported from the Canadian Shield [FRAPE].

brine to be consistent with the influx of sedimentary brine along fractures from the overlying Cambrian sediments at some time in the past, and subsequent modification by interaction with the granite.

\section{Elution of Tritium through the Core}

By pumping tritiated water through the core, we can, in principle, obtain a measure of the effective pore volume. The core described in the preceding report in this series [STEINDLER-1981C] was used. The core and equipment were disassembled and dried before the experiment, so that there was no water in the apparatus between the injection valve and the outlet from the core.

A $10-\mu \mathrm{L}$ spike of water labeled with $0.90 \mathrm{nCi}(33.3 \mathrm{~Bq})$ of tritium was injected into the stream of deionized water pumped into the core. The effluent was collected and analyzed for tritium.

The results are shown in Fig. 24. The flow rate could not be monitored during the experiment, but it was steady at $0.010 \mathrm{~mL} / \mathrm{h}$ before the injection ( confining pressure $=8.5 \mathrm{MPa}$, pressure drop across core $=3.4 \mathrm{MPa}$ ). There is a peak at approximately $5-10 \mathrm{~h}$, which corresponds to $50-100 \mu \mathrm{L}$. This corresponds approximately to an effective porosity of less than or equal to 


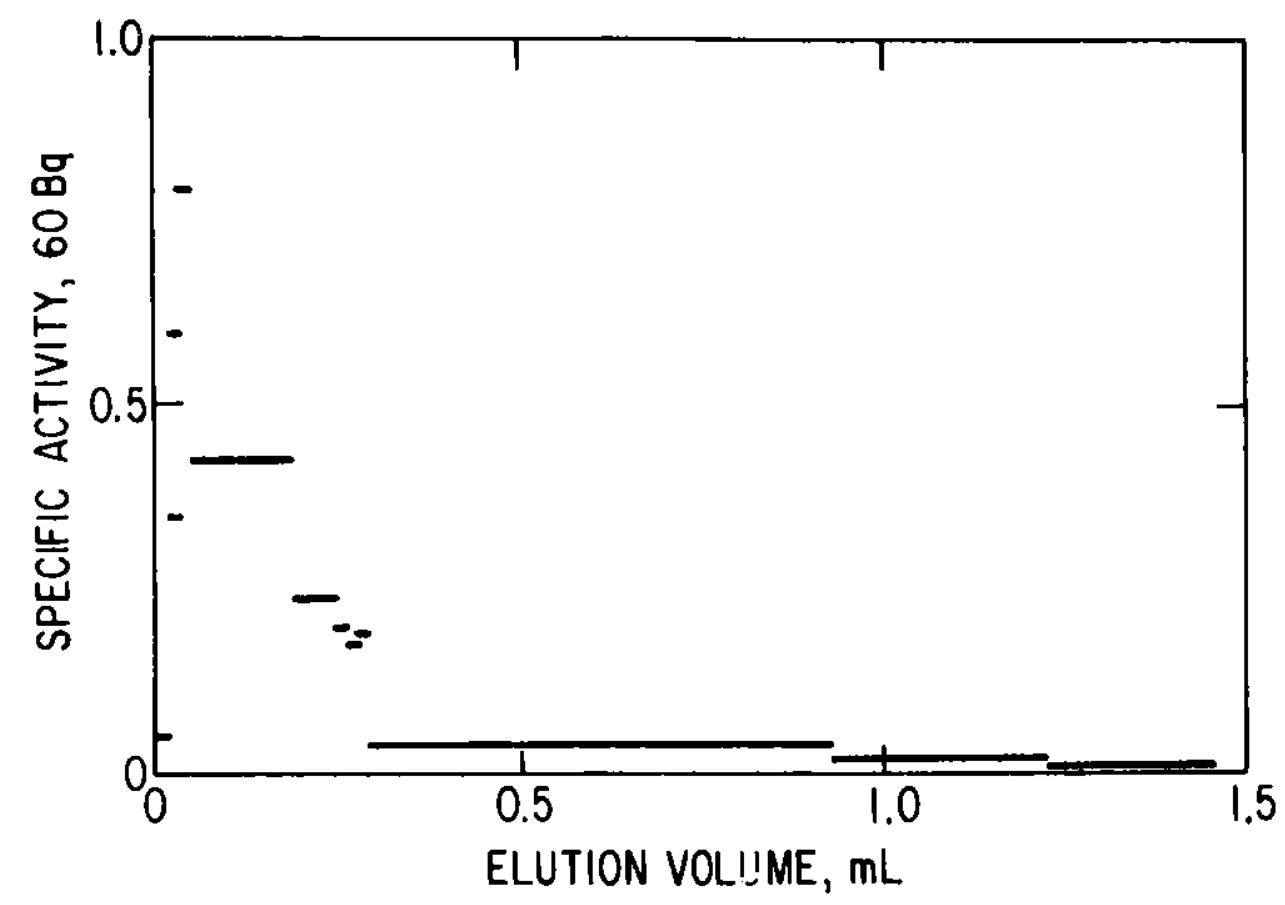

Fig. 24. Elution of Tritium from Granite Core

0.00033 to 0.00067 . This is approximately one order of magnitude lower than the measured total porosity of 0.005 . Since the volume is very small and there is a possibility of a substantial volume in the gaps between the rock and the end plates, a more exact analysis would be difficult.

On 1 y $43 \%$ of the tritium was recovered in $1.5 \mathrm{~mL}$ of flow. The remainder presumably diffused into the rock.

If the effective flow porosity were larger, it would be feasible to obtain an estimate of effective porosity (more correctly, we obtain a single parameter which includes effective crack width and effective diffusion coefficient) as a function of flow rate. This could be related to initial concentration in the effluent. In practice, we will have to polish the ends of the rock quite $\mathrm{flat}$ in order to minimize end volume.

We can do a mass balance calculation which is instructive. A total of $2.4 \times 10^{-4} \mathrm{~mol}$ of $\mathrm{Cl}^{-}$was eluated in $10.3 \mathrm{~mL}, 10 \%$ of it in the first $0.10 \mathrm{~mL}$ [STEINDLER-1981C]. If we have an effective pore volume of $\leq 0.1 \mathrm{~mL}$, the initial $\mathrm{Cl}^{-}$concentration was at least $\frac{0.1\left(2.4 \times 10^{-4}\right)}{10^{-4}}=0.24 \mathrm{M}$. Guessing a total porosity of 0.005 and a core volume of $149 \mathrm{~mL}$, the concentration of $\mathrm{Cl}^{-}$ was at least $0.31 \mathrm{M}$. This means that a large fraction--about $10 \%$ or more--of the salt in the rock was eluted. 


\section{Improved Sampling Techniques}

Unfortunately, the pore volume observed by tritium elution is rather small and is rather close to the unavoldable void volume formed between the rock and the core holder end plate. In this case, we find it difficult to obtain an unambiguous elution curve. There are several good ways of solving this problem.

\section{Tracers}

A very useful method for determining the pore water volume is to use labeled $\mathrm{H}_{2} \mathrm{O}$ as the eluent. We will probably use trit.lum tracer. If the first fraction had a $\mathrm{NaCl}$ concentration of, say, $0.1 \underline{M}$ and an unlabeled water concentration of $10 \%$, then the $\mathrm{NaCl}$ concentration could presumed to be $0.1 / 0.1=1 \mathrm{M}$.

\section{Elution by Gas or Immiscible Liquid}

Inert gas may be suitable as the eluent. The advantages are that the samples would be of excellent quality and easy to obtain. The disadvantage is that it might not work.

Immiscible liquids should be very effective eluents. The disadvantage is possible slight contamination of the sample by the immiscible liquid.

3. Porosity Measurement and Leaching

The salt concentration can be determined from the amount leached and the effective porosity. The amount leached can be determined by flow through a core (which may be dry) or by leaching of crushed material. Either method is particularly useful for dried core samples. It is likely that the cores from UPH-3 had already dried out by the time they were received.

Information on porosity should be obtainable by weighing cores before and after elution and after drying in an oven. High precision in weighing is required.

E. Drying of Samples

The UPH-3 samples were not fresh as recelved, and so, aside from being stored in plastic bags, no measures were taken to prevent further drying.

We can estimate how fast a core w111 dry. A good approximation assumes isothermal diffusion of water vapor, since evaporation does not cool a large rock mass very much. We assume as an approximation that the relative humidity is $100 \%$ at the water/vapor interface and $50 \%$ at the core surface.

The equation in cylindrical coordinates for diffusive flux, F, through an infinite cylinder of rock is

$$
F=\frac{-D \varepsilon \delta}{\tau^{2}} \frac{\partial c}{\partial r}
$$


where $c=$ concentration, $\varepsilon=$ porosity, $D=$ diffusion coefficient, and $\delta / \tau^{2}$ is a geometrical factor characteristic of the rock matrix. Let

$r_{f}=$ position of weter/vapor interface

$r_{0}=$ radius of cylinder.

From the concentration of pure 1 iquid water

$$
F=-0.0555 \& \mathrm{~d} \mathbf{r}_{\mathrm{f}} / \mathrm{dt} ;
$$

$F$ is given in terms of $\mathrm{mol} \cdot \mathrm{cm}^{-2} \cdot \mathrm{s}^{-1}$.

For steady state diffusion, the gradient $\frac{\partial c}{\partial r}$ is nearly constant and will be assumed to be so. The concentration of water vapor in saturated air at $22^{\circ} \mathrm{C}$ is $1.08 \times 10^{-6} \mathrm{~mol} / \mathrm{cm}^{3}$. Then

$$
\frac{d c}{d r}=\frac{1.08 \times 10^{-6}}{2\left(r_{f}-r_{o}\right)}, r_{f} \leq r \leq r_{o}
$$

Combining Eqs. 1 and 2 , and 3 , we obtain

$$
\begin{aligned}
& \frac{d r_{f}}{d t}=\frac{1.08\left(10^{-6}\right)}{0.0555} \frac{D \delta}{2 \tau^{2}} \frac{1}{\left(r_{f}-r_{o}\right)} \\
& r_{f}-r_{o}=4.4\left(10^{-3}\right)\left(\frac{D \delta}{\tau^{2}} t\right)^{1 / 2}
\end{aligned}
$$

Using a reasonable value of $\delta / \tau^{2}=0.5$ [NERETNIEKS] and $D=0.25 \mathrm{~cm}^{2} / \mathrm{s}$, $r_{f}-r_{o}=0.00156 \sqrt{t}$, where $r_{f}$ is in $\mathrm{cm}$ and $t$ is in seconds.

That means that a $4.7-\mathrm{cm}-$ diameter rock core may dry out in as short a time as 28 days. Thus, it is very important that rocks be protected against evaporation after 8 ampling.

\section{F. Migration during Intrusive Emplacement}

This is a summary of the implication of the int rusive processes that have been studied in relation to nuclear repository safety assessments. The Jurassic Notch Peak Stock, House Range, Utah, is a granitic intrusion in carbonate strata. The hot magmatic emplacement of the porphyrit ic quartz monzonite may show processes analogous to those expected from a nuclear waste repos itory. In particular, migration of trace elements from the stock may be related to the possible dispersal of radionuclides from a nuclear waste repository. Skarns occur around the stock at contacts between the monzonite and strata; these are obviously formed by trace-element migration, as well as major-element migration. In this analysis, however, we are interested in migration through the volume of the rock strata which are host to the intrusion. 
Evidence to data indicates no perceptible migration of trace elements from the stock. Most of the recent information has been summarlzed by [HOVER] and discussed by [COUTURE]. A large portion of the variance seen in the composition of samples taken at different distances from the stock resulted from mixing of the argillaceous and calcium carbonate components of the strata during deposition. Only the enrichment of rubidium relative to potassium in the higher grade samples is a possible migration-produced phenomenon. Considerably more work would be required to establish even this minor phenomenon as migration-produced.

The results suggest no new processes of migration that would need to be considered in the design of a nuclear waste repository.

A more 11kely structure to exhdbit trace-element migration would be a kimberlite pipe of South Dakota. The pipes involve the intrusion of melts that are much more fluid (containing more water and alkalis) than the monzonite intrusion, and this would be expected to lead to migration. In studying migration from these kimberlite pipes, its applicability, if any, to the migration processes at a nuclear waste repository would have to be considered. 


\section{LIGHT WATER BREEDER REACTOR PROOF-OF-BREEUING ANALYTICAL SUPPORT PROJECT}

(N. M. Levitz, R. E. Brock, T. F. Cannon, G. L. Chapman, P. G. Deeken, J. E. Fagan, Henry Lautermilch, R. J. Meyer, R. E. Nelson, John Osudar,* J. E. Parks, L. E. Trevorrow, C. G. Wach, I. O. Winsch, and D. G. Graczyk)

A. Full-Scale Shear (FSS)

(P. G. Deeken, J. E. Fagan, and C. G. Wach)

Installation of the full-scale shear (FSS) in shielded hot Cel1 $\mathrm{N}-3$, scheduled for this quarter, was deferred as a result of an ongoing program review by DOE of the draft ANL Project Plan accommodating the 3-y extension of LWBR operations. Installation is expected to proceed after plans reflecting the extended project schedule are approved. During the present period, the shear remained in a mockup area adjacent to the shielded cell facility, in short-term storage. Shear and feed system components and modules were removed, boxed, and stored, and the machine base assembly was wrapped and a nitrogen purge provided to minimize corrosion of the feed system bearings, ways, and ball screw.

The shear control and instrumentation consoles were reworked during this period to incorporate the numerous changes and additional instrumentation which had been added on a trial basis to the shear and consoles during the previous out-of-cell testing (refer to [STEINDLER-1981]). The computer interface module (CAMAC) was located in a separate, third console to minimize electrical noise problems. The CAMAC was fully rewired to the shear controls. Also, feed-through cables and wire harnesses for the added instrumentation that will pass through cell penetrations to the shear were fabricated and wired into the existing cell junction boxes.

Some minor rework on the previously installed in-cell equipment was completed. The enclosure mercury vapor lamps we re modified to pernit remote lamp replacement in the event of lamp failure. The fuel rod entry port on the alpha-enclosure side wall was modified to ease the handling of the IN-40 (fuel rod secondary) container when used to deliver a fuel rod to Cell $\mathrm{M}-3$. Also, an extended-reach manipulator was refurbished by hot-cell personnel to complete Cell M-3's complement of eight master-slave manipulators.

Modifications were also made to the IN-40 extractor device, which removes the container contalning the fuel rod from the shipping cask and delivers it to Cell $\mathrm{M}-3$. Following these modifications (again made to improve remotehandling capability), a dry run of the fuel rod delivery procedure was completed successfully.

Documentation of the shear's design and operation was begun, and a draft version completed. A summary of this document was sent to the American Nuclear society for possible presentation at the Kemote Technology Seminar in November, 1981 .

\footnotetext{
ॠ Sclence Applications, Inc., Oak Brook, IL.
} 
B. Single Unit Dissolver (SUD)

(I. O. Winsch, T. F. Cannon, Henry Lautermilch, and R. E. Brock)

A series of water and acid bollup tests was successfully completed, using the new single unit dissolver (SUD). The new SUD, consisting of a tantalum alloy primary vessel within a stainless steel secondary vessel, incorporates an external heater design, rather than the annular heater used in the iritial SUI design. The annular heaters, which were wrapped on the tantalum vessel, experienced repeated fallures. This, coupled with the difficulty in remotely replacing the heater elements, led to the external heater design in which 18 cartridge heaters are mounted in wells around the secondary vessel. A helium atmosphere between the primary and secondary vessels is used to enhance heat transfer.

In the dozen bollup tests completed using the external heaters, no significant increases in the time required to reach operating temperature $\left(195^{\circ} \mathrm{C}\right)$ and pressure $(860 \mathrm{kPa}$ or $125 \mathrm{psig}$ ) were observed. In one test, a 3-L charge of Thorex was brought to vigorous boiling conditions in approximately $40 \mathrm{~min}$ (indicated by an accelerometer-audio system attached to the dissolver). Wall temperatures of the stainless steel outer vessel were found to be $325-334^{\circ} \mathrm{C}$. The acid was afterward cooled to $80^{\circ} \mathrm{C}$ (nominal temperature before transfer to the blend tank), using a $19 \mathrm{Ls}^{-1}(40-\mathrm{cfm})$ blower directed at the sUD. Cooldown time was approximately $1.25 \mathrm{~h}$. The other bollup tests conducted, in which various amounts of acid or water charges were used, confirmed these results. During these tests, the four SUD heater temperature-controllers were calibrated. All heaters now operate within several degrees of each other.

Plans for future SUD testing have been prepared, including dissolution tests using unirradiated $\mathrm{ThO}_{2}$ charges as a stand-in for fuel. Samples of sheared $\mathrm{ThO}_{2}$, including $\mathrm{Z1rcaloy}$ hulls, have been prepared to simulate the dissolution of $35.5-\mathrm{cm}(14-i \mathrm{n}$.$) segments of blanket rod. Additionally,$ cross-contamination studies using $\mathrm{U}_{3} \mathrm{O}_{8}$ as a tracer will be performed to determine the extent of contamination from run to run, and to aid in developing dissolver and blend tank decontamination procedures.

Also planned are corrosion studies using coupons made of the tantalum alloy used in fabricating the SUD primary vessel. These tests will involve placement of the coupons in the liquid and vapor reglons of the dissolver during actual dissolutions. Data from these tests are to be used in determining an expectej primary vessel lifetime.

Selection of the methods and materials to be used in sampling the dissolver solution is also in progress. A procedure for sampling the dissolver solution, after the second dissolution (for dissolution of residual fuel) w.s tested successfully. The method uses a disposable sampler connected to a vacuum source, and draws samples from the transfer line between the dissolver and the blend tank. The selection of vials to be used to store archive samples 18 under review (plastic vials used in the pilot-scale program falled due to radiation exposure). At present, plastic-coated glass vials [STEINDLER-1981C] appear to be satisfactory for this purpose. 
The temperature-control system ( $T C S$ ), installed in the SUD hot cell to maintain the electronic balance temperature within specified operating limits, performed satisfactorily in recent tests. Tests included determination of the effects of temperature changes on the balance performance and calibration. The balance, a Mettler PS-15, is used to support and welgh the blend tank assembly in the cell. Cooling of the balance is required because the blend tank assembly is heated during sampling and spiking. Results will provide input for the design of the multiple-dissolver system. These tests showed that the TCS maintained the stability of the balance output over the operating temperature range.

C. Multiple-Dissolver System (MDS)

(I.0. Winsch, T F. Cannon, Henry Lautermilch, and K. E. Brock)

Design work continued at ANL-ENG on the multiple-dissolver system (MDS). A package with specifications for procurement of the dissolver condenser cooling system and piping was completed, as were installation drawings for the various systems such as condenser cooling, off-gas, and acid transfer; also, design of the waste tanks and off-gas tanks was completed. Design work was halted on June 15 for review of the Project, with the system design about two-thirds complete. Acceptability of the four tantalum primary dissolver vessels [STEINDLER-1981C] w1ll be established next quarter after additional corrosion data are received.

Exploratory testing of the effects of radiation on electronic balances was carried out. The purpose of these tests, done in cooperation with the Mettler Instrument Co. of Highstown, NJ, was to identify the radiation susceptibility of the Model PK-60 balance. This balance has been proposed for use in weighing the blend tank assemblies in the MDS. (The Mettler PS-15 balance, used in the SUD prototype will no longer be used.) The balance was tested in the cobalt-60 Irradiation Facility at ANL. It was found that the balance is susceptible to radiation damage; after a dose of $8000 \mathrm{R}$, the balance stupped functioning completely.

An examination of the electronic balance revealed that three components had been damaged by the gamma radiation. The falled components were three IC (integrated circuit) chips: a KOM (read-only memory), a 2-80 microprocessor, and a MOS (metal-oxide semiconductor). Recent tests of radiation effects on electronic components done at the Jet Propulsion Laboratory In Pasadena, CA, indicate that these particular components will fail after an accumulated dose of approximately $3000 \mathrm{R}$. Thus, it appears necessary that the balance electronics be located outside the hot cell. Plans to modify our balances are being made.

\section{Scrap and Waste}

(L. E. Trevorrow and R. E. Nelson)

Planning for the disposal of LWBR Proof-of-Breeding (POB) residues had been focused on converting the dissolver solution to solids at ANL by spray calcination followed by shipment to the Kockwell-hanford ( $R-H)$ storage facllity. Recently, however, in an effort to reduce costs, DOE-HQ has been encouraging shipment of these solutions in liquid form to another DOE contractor if shipment is feasible. Furthermore, DOE-HQ has recommended that 
the procurement of caicination equipment be deferred until a review of waste form options is made. As a result, activities on the disposal of the dissolver solutions have been restricted to finishing the design of the spray calciner, studying the effectiveness of ferric oxide for calcined of $f$-gas traps, and studying the feasibility of shipping the dissolver solutions in liquid form to another site. Progress in these activities is briefly summarized here.

Effort on preparing to calcine dissolver solutions consisted matily of detailing design layouts from which fabrication drawings of equipment may be made. These efforts have been focused on equipment items for a portion of the system that would permit calcination tests of nonradioactive solutions. This portion of the system is being referred to as the mockup. The items include: calciner, scrubber, feed tank, ruthenium traps, mounting rack, drip pan, and associated prefabricated components of the piping and instrumentation system. For the items that can be used for calcination tests, the design phase is nearing completion. The final design review of this equipment is to consist of a series of reviews of individual items or small groups of associated items. To date, Chemical Engineering Division (CEN) and ANL-ENG personnel completed and approved the design of the scrubber and feed tank, making only relatively minor changes. Review of the remaining items is expected to be completed next quarter.

Ferric oxide is the intended active ingredient in traps for removal of ruthenium from calciner off-gas. The behavior of ferric oxide on exposure to simulated calciner off-gas was tested. Since off-gas from calcination of the LWBR analytical residues will have an unusually high nitric acid concentration and may contain HF, it is important to determine whether these components will cause any significant physical effects (e.g., caking or plugging) or chemical changes of the trap media. In tests of duration up to $16 \mathrm{~h}$ at $250^{\circ} \mathrm{C}$, the media showed no plugging or caking. Although changes in color occurred, analyses showed that the 1 ron content of the bed remained unchanged. It was concluded that no loss of the active ingredient by exposure to the acid-air mixture should be expected. The color change remains unexplained.

Calculations by the ANL Office of Health and Safety (W. Lipton) have verified the need to incorporate surge vessels in the calciner off-gas train to slow the velocity of the gas and to permit decay of radon-220 within the shielded area. Current plans are to provide several segments of $30-\mathrm{cm}-\mathrm{dia}$ (12-in.-dia) pipe for this purpose. The solid daughters (decay products) of radon-220, which have a controlling half-life of about $11 \mathrm{~h}$ and high-energy gammas ( $\leq 2.6 \mathrm{NeV}$ ) would collect in these segments of plpe (located in the cell).

The reduction of costs for disposal of the dissolver residues has been emphasized recently by DOE-HQ in their recent request for the preparation of a "position" paper summarlzing conclusions on the comparison of alternative methods of disposal of LWBR dissolver residues. As reported previously [STEINDLEK-1981C], it is requested that shipping the dissolver residues in the form of liquids to another DOE contractor is to be considered in this study, as the primary alternative to the original plan of calcination at ANL followed by shipment of the powdered calcine to Rockwe1l-llanford. Shipment of liquids to another contractor who could store liquid or who has calcination 
equipment already installed has been suggested by DOE-liQ as a possible means of reducing costs. This practice would require (a) compliance with applicable regulations, (b) a DOE contractor capable of dealing with the liquids, and (c) the availability of licensed transport equipment, compatible with the characteristics of the LWBR residues.

The DOE Manual indicates that the liquids could be shipped if they are in compliance with the Manual.

With regard to acceptance of liquid by other DOE contractors, a previous survey indicated reluctance, but recent communications between $\mathrm{NOE}-\mathrm{CH}$ and other field of fices indicate that the Savannah River Plant (SRP), Idaho Nuclear (ID), and Battelle Pacific Northwest Laboratory (PNL) are at least willing to consider this scheme.

DOE-CH is presently reviewing the directories of DOE-licensed equipment to determine the avallability of transport equipment that is suitable for shipping the LWBR dissolver solutions. Important considerations in any scheme that involves transport of liquids are the needs and costs for equipment to transfer liquid from the transport vessels to receiver's equipment that would be used for storage or processing.

E. Computer System

(J. E. Parks and J. E. Fagan)

An integrated computer system is being developed which will provide automatic data acquisition, process monitoring capabilities, and some automatic process control. Currently, computer system hardware consists of a VAX-11/780* (shared with other Chemical Englneering Division users) and a PDP-11/23.* The latter unit is connected to the FSS and the SUD via standard CAMAC interfaces. Work on the computer system during the report period is summarized below.

\section{Hardware}

The Interface between the PDP-11/23 and the SUD controls was completed, except for sensors associated with the dissolver heaters. Debugging of the interface between the computer and the two balances in Cell K-3 continues. The linkage between the $\mathrm{VAX}-11 / 780$ and the $\mathrm{PDP}-11 / 23$ was connected, and part of the software to implement data transfer was installed.

\section{Software}

Software developments fall into two areas-general applications software for the Project computer system, and programs for operating the SUD. General applications sof tware completed during this quarter include the following:

EDITUR - a program to permit entering and modifying the operating procedures for the Project operating systems (presently the FSS and SUD), and

ॠProducts of Vigital Equipment Corp. (DEC), Maynard, Mass. 
MONITR - a program which will continually monttor all Project sensors and controls and which will transfer pertinent data to storage.

Ongoing software efforts include programs for intercomputer data transfer and interprogram communication within the PDP-11/23.

Operating programs completed for the SUD include those programs which will replace the function of the NODICON, a programmable controller [STEINDLER-1981B] and several of the specific steps in the sUD operating procedure. These procedure steps (dissolver leak test,, blend tank leak test) have been used to familiarize the SUD operators with computer operations.

updated.

Technical documentation of the Project computer system has been

F. Eirror Analysis

(D. G. Graczyk and J. E. Parks)

A comprehensive error analysis for the $L W B K-P O B$ Project is in preparation. During this work, it was found that a contribution to the possible blas error in the uranium assays had been overlooked in a previous treatment [ANL]. This bias contribution arises from uncertainty in the $235 \mathrm{U}-\mathrm{to}_{0}-238 \mathrm{U}$ ratio of the NBS U-500 $j$ sotopic standard reference material (SRM). Copies of a memorandum summarizing the mathematical treatment of this error source and change pages reflecting the consequent revisions to the VR report were forwarded to $B A P L$ for review. This revised treatment was promptly and thoroughly reviewed by BAPL personnel, who carrled out a rigorous mathematical analysis of the bias contributions. The BAPL analysis gave expressions which yielded slightly different estimates of bias error than did the ANL treatment. Upon investigation, it was found that an algebraic error had been made in the ANL treatment. In the course of rectifying this error, it was noted that the relationship between bias error in the uranfum assays and uncertalnty in the U-500 isotopic standard is influenced by the mass dependence of the correction factors on the mass spectrometrically determined 1sotope ratios. This development, which did not affect earlier results, has been incorporated into Alv's error analysis; final revision and review of the Validation Rod (VR) report is under way.

G. Analytical

(R. J. Meyer)

The preshipment testing and inspection of the mass spectrometer at V. G. Isotopes, Winsford, England, were completed on June 27, 1981, and approval to ship the instrument to ANL was granted.

All components of the Instrument function properly, and instrument specification parameters such as peak shape, peak top flatness, and abundance sensitivity were within ANL speciflcations. The precision obtained on 15 analyses of NBS U-500 uranium standard, $\pm 0.04 \%$, was slightly outside ANL specifications of $\pm 0.03 \%$. Th1s deviation is not considered significant since the operator's sample loading technique was not optimized. It is anticipated that after installation at ANL, where analytical conditions can be more carefully controlled, all specifications will be met. 


\section{REFERENCES}

ANL

Report on the Shearing, Dissolution, and Analysis of GRIP-II Rod 79-453

Validation Rod, Argonne National Laboratory Report ANL-80-88 (March 1980).

Bond

D. C. Bond, Hydrodynamics in Deep Aquifers of the Illinois Bas in, Ill.

Geol. Surv. Circ. 470 (1972).

Cornman

W. R. Cornman, Composite Quarterly Technical Report, Jong-Term High-Level Waste Technology, October-December 1979, p. 52, DP-79-159-4 (1980).

Couture

R. Couture, Argonne National Laboratory, letter to J. J. Papike

(February 17, 1981).

Frape

S. K. Frape and P. Fritz, A Preliminary Report on the Occurrence and Geochemistry of Saline Groundwaters on the Canadian Shield, Atomic Energy of Canada, Limited, TR-136 (1981).

Fritz

P. Fritz and T. J. Reardon, Isotopic and Chemical Characteristics of Mine Waters in the Sudbury Area, Atomic Energy of Canada, Limited, AECL-TR-35 (1979).

Gephart

R. E. Gephart, R. C. Arnett, R. G. Baca, L. D. Leonhart, and

F. A. Spane, Jr., Hydrologic Studies with the Columbia Plateau,

Washington, Rockwe 11 Internat ional Report RHO-BWI-ST-5 (October 1979).

Hover

V. Hover, J. J. Papike, P. Nabelek, and J. C. Laul, The Notch Peak

Contact Metamorph ic Aureole, Utah, Eos Magazine, 62(17), 434

(April 28, 1981).

Illino is

Illinois State Water Survey and Hittman Associates, Feasibility Study on Desalting Brackish Water from the Mt. Simon Aquifier in Northeastern Illinois, Ill. State Water Surv,, Contract Publ. 153 (1973).

Johnson- 1961

E. R. Johnson and J. Forten, Radiation Induced Decomposition of Inorganic Nitrates, Discuss. Faraday Soc. 13, 238 (1961).

Johnson-1970

E. R. Johnson, The Radiation Induced Decomposition of Inorganic

Molecular Ions, Gordon and Breach, Science Publisher, Inc., New York (1970). 
Mecham

W. J. Mecham, L. J. Jardine, R. H. Pelto, G. T. Reedy, and

M. J. Steindler, Interim Report of Brittle-Fracture Impact Studies: Development of Methodology, Argonne National Laboratory Report ANL-81-27 (August 1981).

Mende I

J. E. Mendel et al., Annual Report on the Characteristics of High-Level Waste Glasses, Battel le Northwest Laboratories Report BNWL-2252, p. 5 (June 1977).

Meents

W. F. Meent 8, A. H. Bell, O. W. Rees, and W. G. Tilbury, Illinois Oil-Field Brines: Their Geologic Occurrence and Chemical Composition, I11. Petroleum, No. 66 .

Neretnieks

I. Neretnieks, Diffusion in the Rock Matrix: An Important Factor in Radionuclide Retardation, J. Geophys. Res. 85B, 4379-4397 (1980).

SRL-1980A

Savannah River Laboratory, Waste Management Program Technical Progress

Report, January-March 1980, DP-80-125-1, P. 39 (September 1980).

SRL-1980B

Savannah River Laboratory, Waste Management Program Technical Progress

Report, January-March 1980, DP-80-125-2 (December 1980).

Ste ind ler-1981A

M. J. Steindler et al., Chemical Engineering Division, Fuel Cycle Section, Quarterly Progress Report, July-September 1980, Argonne National Laboratory Report ANL-80-114.

Ste ind ler-1981B

M. J. Steindler et al., Chemical Engineering Division, Fuel Cycle Section, Quarter 1y Progress Report, October-December 1980, Argonne National Laboratory Report ANL-81-13.

Steind ler-1981C

M. J. Steindler et al., Chemical Engineering Division, Fuel Cycle Section, Quarterly Progress Report, January-March 1981, Argonne National Laboratory Report ANL-81-35.

WNYNSCS

Western New York Nuclear Services Center Study, Vol. 2, Companion Report, TID-28905-3.

Zeleny

R. A. Zeleny and E. L. Piret, Description of Energy in Single-Particle Crushing, Ind. Eng. Chem. Process Des. Dev. 1, 34-41 (1962). 